Bulletin of the Natural History Museum, 2017, 10: 57-104.

Received 21 Jun 2017; Accepted 17 Sep 2017.

doi:10.5937/bnhmb1710057D

UDC: 069.51:597.6/598.1(497.11); 57/59:005.71(497.11)

Original scientific paper

\title{
THE HERPETOLOGICAL COLLECTION OF THE INSTITUTE FOR BIOLOGICAL RESEARCH "SINIŠA STANKOVIĆ", UNIVERSITY OF BELGRADE
}

\author{
GEORG DŽUKIĆ ${ }^{1}$, LJILJANA TOMOVIĆ ${ }^{2}$, MARKO ANĐELKOVIĆ ${ }^{1}$, \\ ALEKSANDAR UROŠEVIĆ ${ }^{1}$, SONJA NIKOLIĆ ${ }^{2}$, MILOŠ KALEZIĆ ${ }^{1}$ \\ ${ }^{1}$ University of Belgrade, Institute for Biological Research "Siniša Stanković", \\ Bulevar Despota Stefana 142, 11000 Belgrade, Serbia \\ ${ }^{2}$ University of Belgrade, Faculty of Biology, Institute of Zoology, Studentski trg 16, \\ 11000 Belgrade, Serbia, e-mail: lili@bio.bg.ac.rs
}

Key words: Reptiles, collection

\section{INTRODUCTION}

The history of the Herpetological collection of the Institute for Biological Research "Siniša Stanković" University of Belgrade is 80 years long. The collection was initially formed from private donations and by collecting new samples in the field. Also, the herpetological collection of the Institute of Zoology, Faculty of Natural Sciences University of Belgrade was added to it. This early collection consisted of samples from ex-Yugoslavia. Unfortunately, large parts of the initial collection suffered an immeasurable damage during the Second World War: most of the 
documentation and a large number of specimens were destroyed. During the professional career of Professor Milutin Radovanović (1900-1968), the collection was expanded. His seminal work has laid foundations of herpetology in the entire region, together with acquisition and organizing of the material in the collection. His contribution prior to 1941 included samples from islands of the Adriatic Sea (present-day Croatia).

After the Second World War, Professor Radovanović resumed the acquisition of the material - again mostly from the territory of present-day Croatia, but some samples were also collected from Serbia, Montenegro and Macedonia. Another important contributor was Professor Gojko Pasuljević (1931-1997), who contributed the specimens of Ablepharus kitaibelii and Vipera ammodytes from the province of Kosovo and Metohija, Serbia.

The next period of intensive acquisition of specimens started after 1968, with large population samples being deposited in the herpetological collection by the first author (G. Dž.). Since then, large amounts of material were being added to the collection. However, apart from assigning collection numbers to the specimens, there were no systematic efforts at their sorting and cataloguing. During the inventory undertaken between 2011 and 2015, the Batrachological collection was separated from the herpetological material, and it was published independently (Džukić et al. 2015).

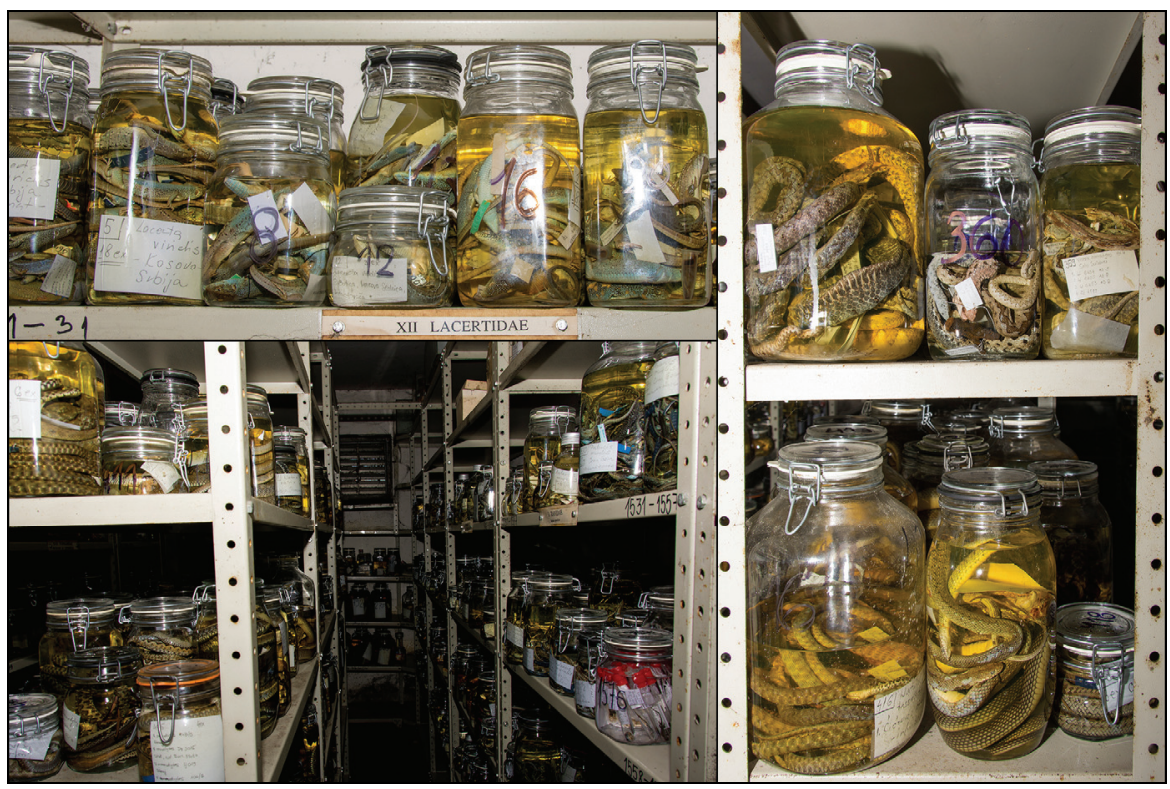

Fig. 1. - Herpetological collection of the Institute for Biological Research "Siniša Stanković" University of Belgrade, Belgrade, Serbia. 
The Herpetological collection of the Institute for Biological Research currently contains 8213 specimens originating from 23 countries (Fig. 1). Most of the specimens were collected in the territory of the former Yugoslavia, with Serbia and Montenegro being especially well represented. The collection is supplemented with specimens belonging to several countries from Asia, Africa, North and South America, as well as several European countries. The Herpetological collection contains several subcollections of the herpetologists or a student association who significantly contributed with specimens or with distribution records. These sub-collections are as follow: Georg Džukić, Milutin Radovanović, Gojko Pasuljević, Ljiljana Tomović, Jozsef Mikuska, Jelka Crnobrnja Isailović, Rastko Ajtić, Imre Krizmanić, Nenad Labus, Sonja Đorđević, Students' research association "Josif Pančić".

\section{MATERIALS AND METHODS}

The collection of reptiles of the Institute for Biological research "Siniša Stanković" consists of herpetological materials preserved in $75 \%$ ethanol. Some specimens had previously been kept in the freezer or stored in formaldehyde, and were recently transferred to $75 \%$ ethanol. All materials are stored in labelled glass jars. During the period of seven years (from 2011 until the end of 2017) the complete sorting, conservation and inventory of the collection has been conducted, and new labels with consecutive catalogue numbers were added to the old ones, with a new collection number assigned to each jar. In some cases, the stored specimens only had collection numbers, so the localities and collection dates needed to be retrieved from field logs or collection protocols. Initially, a lot of specimens were stored in "mixed" jars, containing various species (e.g. all samples from one locality/date or contributed by one legator). After the systematization of all available information, the specimens were stored in single-species jars and grouped by the country and region of origin in order to facilitate their cataloguing and future work with the collection.

For the majority of species, identification was done according to the relevant herpetological literature. In the case of the recently described species (Dinarolacerta montenegrina sp. nov.), the specimens were identified based on the published diagnostic characters and geographic origin of samples (Ljubisavljević et al. 2007). Regarding taxonomy and current nomenclature, we followed Sillero et al. (2014) and Uetz \& Hošek (2017). Anguis fragilis sensu lato was treated as a species complex: the samples in the collection presumably belong to at least three recently described distinct species (Jablonski et al. 2016), but these are difficult to distinguish both morphologically and ecologically, and their geographic 
distribution is still not clearly defined, so we opted for a conservative approach (Tomović et al. 2015a, b).

\section{RESULTS}

\section{Catalogue of taxa and specimens}

\section{Testudines}

\section{CHELONIIDAE}

\section{Caretta caretta}

Montenegro Mediterranean Montenegro Tivat: Lastva Grbaljska, Jaz beach (leg. A. Hegediš, B. Mićković, 10/02/2000, 1940, n.spec. =1).

\section{EMYDIDAE}

\section{Emys orbicularis}

Montenegro Mediterranean Montenegro Ulcinj: Bregvija (leg. G. Džukić, M. Kalezić, 08/06/2005, 1947, n.spec. = 1); i. Vada (leg. anonim. 02/04/1989, 1944, n.spec. =1); Northern Montenegro Bjelasica Mt.: Biogradsko lake, Lalev potok (leg. G. Džukić, M. Kalezić, 24/08/1994, 1944, n.spec. $=1$ ); Submediterranean Montenegro Danilovgrad: Lazine (leg. M. Kalezić, 15/03/2008, 1944, n.spec. =1); Serbia Bačka Sombor: Bački Monoštor (v.) (leg. anonim., 16/ 03/1997, 1945, n.spec. = 1); Banat Bela Crkva: Mrtva Nera (leg. G. Džukić, M. Kalezić, 18/03/2005, 1943, n.spec. =1); Stara Nera, pond at the right side of the road (leg. M. Marković May 1987, 1943, n.spec. $=2$ ); Deliblatska peščara: Bela Crkva - Kovin, $500 \mathrm{~m}$ from crossroad to Kajtasovo (v.) (leg. G. Džukić 23/05/2014, 1946, n.spec. = 1); after crossroad to Kajtasovo (v.) (leg. G. Džukić 05/05/2014, 1946, n.spec. = 1); Ludvig Polje, near crossroad to Utrine (leg. G. Džukić 05/05/2014, 1946, n.spec. = 1; 23/05/2014, 1946); near crossroad to Kajtasovo (v.) (leg. G. Džukić 05/05/2014, 1946, n.spec. = 1); Labudovo okno, across Salaš Bradvarevića (leg. G. Džukić 01/05/2014, 1946, n.spec. = 1); Utrine (leg. G. Džukić, M. Kalezić 22/05/2004, 1947, n.spec. = 1); Vračev Gaj (v.), Tuzla pond, Đavolji most (leg. M. Marković s. d., 1943, n.spec. = 1); Central Serbia Prokuplje: Đol pond (leg. anonim., 12/08/1951, 1945, n.spec. = 1); Eastern Serbia Dimitrovgrad: Poganovo (v.), Borovsko Polje (leg. G. Džukić, M. Kalezić, 21/05/2008, 1947, n.spec. =2); North-eastern Serbia Negotin: Jabukovac (v.), Balta Ursului (leg. G. Džukić, 28/09/2005, 1947, n.spec. =1); Zaječar: Vražogrnac (v.) (leg. G. Džukić, M. Kalezić 25/04/2009, 1947, n.spec. =1); Srem Pećinci: Obrež (v.), Obedska bara pond (leg. M. Radovanović, July 1949, 1945, n.spec. =1); Šumadija Beograd: Čukarica, Košutnjak (leg. anonim., 28/04/1951, 1945, n.spec. = 1).

\section{TESTUDINIDAE}

\section{Testudo graeca}

Macedonia Vardar Zone Štip (leg. anonim., 26/05/1958, 1941, n.spec. =1).

\section{Testudo hermanni}

Macedonia Vardar Zone štip (leg. anonim., 26/05/1958, 1942, n.spec. = 1); Montenegro Mediterranean Montenegro Budva: Buljarica (leg. S. Đorđević, 17/04/2005, 1942, n.spec. $=1$ ); Serbia Central Serbia Prokuplje: Borovnjak hill (leg. anonim., 08/05/1962, 1942, n.spec. $=1)$. 


\section{Lacertilia}

\section{AGAMIDAE}

\section{Agama lionotus}

Kenya Kenya Mt. Tani (r.): steppe region (leg. G. Nonveiller, 1986, 900, n.spec. =1).

\section{Laudakia badakhasana}

Afghanistan Bamyan "Đavolja dolina” (leg. anonim., 04/08/1972, 753, n.spec. = 3); (leg. anonim. 03/08/1972, 753 , n.spec. $=1$ ).

\section{Laudakia caucasica}

Afghanistan Džam (leg. anonim., 08/08/1972, 912, n.spec. = 2); Kala-I-Nau (leg. anonim., 12/08/1972, 912, n.spec. = 2); Samangan Takht-e-Rostam (leg. anonim., 16/08/1972, 912, n.spec. =2).

\section{Stellagama stellio}

Cyprus (leg. Z. Gradojević 11/05/1955, 784, n.spec. =1).

\section{Trapelus ruderatus}

Afghanistan Kabul Guldara (leg. anonim., 21/08/1972, 887, n.spec. = 1); Šivaki (leg. anonim. 21/08/1972, 887 , n.spec. $=1$ ).

\section{ANGUIDAE}

\section{Anguis fragilis complex}

S. I. (leg. anonim. s. d., 1595, n.spec. = 45); Bosnia \& Herzegovina Bosnia Igman Mt.: Veliko Polje (leg. anonim., 01/08/1974, 32, n.spec. =2); Jajce: surrounding (leg. anonim. 23/08/1953, 32, n.spec. =1); Herzegovina Zelengora Mt.: Gornje Bare (leg. V. Majić, 02/09/1977, 32, n.spec. =1); Čemerno - Tjentište, 2 km toward Tjentište (leg. G. Džukić 12/06/1980, 32, n.spec. = 1); Croatia Baranja Haljevo (leg. J. Mikuška, 04/05/1971, 173, n.spec. =1); Gorski Kotar Delnice: Crni Lug (v.), Vela Voda (leg. anonim., 28/08/1963, 173, n.spec. =1); Lokve: Mrzla Vodica (v.), Rogozno (leg. G. Džukić 19/05/1971, 173, n.spec. = 2); Istria Buje (leg. N. Tvrtković, 1973, 171, n.spec. = 2); Rovinj: surrounding (leg. N. Tvrtković 23/06/1976, 171, n.spec. =1); (leg. S. Leiner May 1977, 171, n.spec. =1); Kvarner Krk (i.): Krk, 8 km south-western trom the city (leg. G. Džukić, 17/07/1975, 171, n.spec. =1); Lika Velebit Mt.: Jablanac (v.), Golić hill (leg. N. Tvrtković, 31/08/1976, 171, n.spec. = 1); Paklenica (leg. N. Tvrtković 30/05/1976, 171, n.spec. =1); Predzid (leg. N. Tvrtković 05/07/1975, 171, n.spec. $=1 ; 06 / 07 / 1975,171$ ); Vrhovine: Doljani (v.), Topluša (leg. anonim. 18/10/1961, 173, n.spec. = 1); Donji Babin Potok (v.), Borića Borik (leg. anonim. 11/05/1962, 173, n.spec. = 1); Slavonia Ivanjić Grad: Križ (v.), forest Žutica, between Sava (r.) and highway (leg. N. Tvrtković, 21/05/1979, 173, n.spec. =1); Velika Gorica Turopolje: Peščenica (v.) (leg. F. Perović, March 1975, 173, n.spec. = 1); Zagorje Samobor: Gregurić Breg (leg. D. Pelić, 11/05/1975, 173, n.spec. =1); Zagreb city: botanical garden (leg. D. Pelić, 15/05/1975, 159, n.spec. $=$ 1); Maksimir: hospital "Rebro" (leg. N. Tvrtković 03/04/1975, 159, n.spec. =1); (leg. G. Džukić 20/05/1971, 159, n.spec. = 1); Medvednica (leg. D. Pelić 1976, 159, n.spec. =2); Zelengaj: Kozarčeva street (leg. K. Korić 04/07/1976, 159, n.spec. =2); (leg. anonim. s. d., 45, n.spec. =4); Macedonia Southern Mountains Kožuf Mt.: Konsko (v.) (leg. P. Jakšić, 18/07/1990, 902, n.spec. = 1); Miletkovo (v.), Miletkovo (v.) - Visoka Čuka, road (leg. G. Džukić 15/06/1981, 902, n.spec. =1); (leg. B. Kryštufek s. d., 902, n.spec. =1); South-western Region Galičica Mt.: Trpejci (v.) (leg. G. 
Džukić, 19/09/1978, 902, n.spec. =1); Jablanica Mt. (leg. B. Kryštufek 27/09/2004, 902, n.spec. =1); Pelister Mt.: Begova Češma (leg. B. Petrov 10/05/1975, 902, n.spec. =1); Western Mountains Korab Mt.: Ćos Alija (leg. G. Džukić, Mazlami, 18.09. \& 19.09.1996, 902, n.spec. =1); Montenegro Central Montenegro Danilovgrad: Prekornica Mt., Bukotica, Martinićke lokve (leg. G. Džukić, M. Kalezić, 09/06/1997, 38, n.spec. = 1); Suva Ponikvica, Razmet Do (leg. G. Džukić, M. Kalezić 30/05/2003, 38, n.spec. = 1); Morača (r.) - canyon: Bare (v.), Monastery Morača (leg. Lj. Tomović, $R$. Ajtić 24/07/2000, 38, n.spec. =2); Eastern Montenegro Bjelasica Mt.: Biogradsko jezero Lake (leg. J. Crnobrnja Isailović, 01/06/1996, 172, n.spec. = 1); (leg. Lj. Tomović, R. Ajtić 19/07/2000, 172, n.spec. = 1); (leg. R. Ajtić June 2003, 172, n.spec. = 1); Zekova Glava, toward (leg. Lj. Tomović, R. Ajtić 20/07/2000, 172, n.spec. = 1); Žijovo Mt.: Lijeva Rijeka, Jablan (leg. G. Džukić, M. Kalezić, M. Marković 2004, 172, n.spec. = 1); Mediterranean Montenegro Bar: Sustaš (leg. G. Džukić, M. Kalezić, 13/06/1997, 28, n.spec. = 1); Boka Kotorska: Koštanjica (leg. A. Cetković 27/05/1998, 42, n.spec. = 1); Luštica Peninsula, Krtole (leg. V. Stevanović 04/02/2008, 42, n.spec. =1); Budva: Sveti Stefan (leg. R. Anđus i Daltoni 19/05/1996, 28, n.spec. =2); Gornji Grbalj: Šišići (v.) (leg. G. Džukić, M. Kalezić, M. Marković 02/06/2003, 42, n.spec. = 1); Kotor: Crikvenica, Vinodol (leg. anonim. 20/04/1964, 42, n.spec. = 1); Dobrota (leg. A. Ivanović, M. Kalezić, I. Aleksić 27/05/1995, 42, n.spec. = 1); (leg. I. Aleksić 26/05/1996, 42, n.spec. = 1); Rumija Mt.: Sutorman, bellow the bend toward Bar (leg. G. Džukić, M. Kalezić, M. Marković 02/06/2003, 28, n.spec. =1); Tivat: Gradiošnica (r.), bridge near Gradiošnica (leg. A. Hegediš 14/05/1996, 42, n.spec. = 1); Lastva Grbaljska, Mrčevsko Polje, Jaz (leg. S. Stamenković 27/05/1996, 42, n.spec. =2); Ulcinj: Ada Bojana, Bojana, southern tributary (r.) (leg. I. Aleksić 13/09/1995, 28, n.spec. = 1); bridge (leg. G. Džukić, M. Kalezić, M. Marković 08/06/2005, 28, n.spec. $=1$ ); Northern Montenegro Durmitor Mt.: Crno jezero Lake, Čeline (leg. P. Jakšić, 02/07/1987, 157, n.spec. = 1); Komarnica (r.) - canyon, Klještina (leg. P. Jakšić 24/07/1985, 157, n.spec. = 1); Tara (r.) - canyon: Crna Poda (leg. V. Stevanović 17/05/1984, 157, n.spec. = 1); Tepca (v.), Devojački most (leg. R. Ajtić, M. Langurov 02/07/2001, 157, n.spec. =2); (leg. Lj. Tomović 30/07/2002, 157, n.spec. =1); (leg. P. Jakšić 26/06/1985, 157, n.spec. =2); Submediterranean Montenegro Lovćen Mt.: Bjeloši (v.), Ivanova Korita (leg. G. Džukić, M. Kalezić, M. Marković, 22/06/2004, 4l, n.spec. =1); Njeguši (v.), Bukovica (leg. G. Džukić, M. Kalezić 10/06/1997, 41, n.spec. =1); Resna (v.), Bjelice (leg. G. Džukić, M. Kalezić 10/06/1997, 41, n.spec. =1); Orjen Mt.: Ubli (v.) (leg. G. Džukić, M. Kalezić 28/08/2002, 41, n.spec. = 1); Podgorica: Gornji Crnci (v.) (leg. R. Pulević 25/09/1988, 41, n.spec. =2); Murtovina (leg. K. Ljubisavljević 07/06/2012, 41, n.spec. = 1); Stijena (v.), toward v. Gornji Crnci (leg. G. Džukić, M. Kalezić 09/06/1997, 41, n.spec. =1); Rumija Mt.: Godinje (v.) (leg. G. Džukić, M. Kalezić, M. Marković 31/05/2003, 41, n.spec. =1); Limljani (v.) (leg. G. Džukić, M. Kalezić, M. Marković 31/05/2003, 41, n.spec. =1); Western Montenegro Nikšić: Grahovac (v.), Kotlić (leg. G. Džukić, 15/06/1983, 31, n.spec. = 1); Vilusi (v.), Slivni Do (leg. M. Bulajić 20/05/1984, 31, n.spec. = 1; November 1982, 31); (leg. M. Bulajić July-September 1980, 31, n.spec. = 5); Vučji Do (v.), Vilatovci (leg. G. Džukić, M. Kalezić, M. Marković 26/06/2004, 31, n.spec. = 1); Serbia Bačka Sombor: Doroslovo (v.) (leg. B. Božičić, May 1985, 176, n.spec. =2); Banat Deliblatska peščara: Jasenovo (v.), Jasenovačka šuma (leg. M. Marković, 28/05/2002, 43, n.spec. = 1; August 1998, 43); Vršački breg Mt.: Česta 2, between the pond and Česta 2 (leg. anonim. 08/06/2012, 43, n.spec. =1); Široko Bilo (leg. anonim. 09/06/1970, 43, n.spec. = 1; 11/06/1970, 43); mountain house (leg. Đ. Novak 14/06/2004, 43, n.spec. = 1); (leg. G. Džukić 03.06. \& 05.06.2009, 43, n.spec. $=2$ ); Central Serbia Goč Mt.: Ćelauša, before the lake (leg. V. Kalafatić, 11/05/1990, 866, n.spec. =1); Jastrebac Mt.: Loparda (leg. anonim. 26/06/1987, 866, n.spec. =2; 30/06/1987, 866); Prokopačka Kosa (leg. B. Božičić 28/06/1979, 866, n.spec. = 2); Ravnište (leg. Z. Dunđerski 03/06/1982, 866, n.spec. $=1 ; 28 / 04 / 1983,866$ ); Kopaonik Mt.: Jošanička Banja (leg. O. Glišić 01/06/1986, 866, n.spec. = 1); Samokovska Reka (r.) - gorge (leg. J. Crnobrnja 01/06/1986, 866, n.spec. =2); Eastern Serbia Dimitrovgrad: city (leg. G. Džukić, M. Kalezić, 21/05/2008, 40, n.spec. = 1); Ozren Mt.: Soko Banja (leg. P. Jakšić 30/06/1973, 40, n.spec. =1); Stara planina Mt.: Ponor (leg. G. Džukić, M. Kalezić 03/07/2008, 40, n.spec. = 1); Toplodolske livade (leg. P. Jakšić 14/07/1991, 40, n.spec. =1); Kosovo Novo Brdo hill (leg. P. Jakšić, 29/05/1985, 39, n.spec. =1); Šar planina Mts.: Brezovica, Durlov potok (leg. A. Rohalj 16/07/1995, 39, n.spec. =1); Metohia Đakovica: Babaj Boks (v.), Beli Drim (r.) (leg. G. Džukić, 18/07/1980, 39, n.spec. =1); Peć: Miliševac spring (leg. P. Jakšić 16/08/1985, 39, n.spec. = 4); North-eastern Serbia Beljanica Mt. (leg. R. Ajtić, July 1998, 44, n.spec. = 1); Bor: Gornjane (v.) (leg. K. Bajić 08/07/1990, 44, n.spec. =1); Kučajske planine Mts.: Sisevac (v.) (leg. A. Stevanović June 1976, 44, n.spec. = 1); Kučevo: Blagojev 
Kamen (v.) (leg. anonim. 07/06/1972, 44, n.spec. = 1); (leg. D. Pavičević 08/05/1988, 44, n.spec. = 1); Paraćin: Grza - gorge (r.), mountain house (leg. Đ. Marković 15/07/2009, 44, n.spec. =1); North-western Serbia Ljig: Čibutkovica (v.), Vinogradina (leg. V. Kalafatić, 29/04/1990, 880, n.spec. $=$ 1); Mali Zvornik: Gornja Trešnjica (v.) (leg. J. Crnobrnja Isailović 05/04/1998, 33, n.spec. = 1); Maljen Mt.: Divčibare (leg. M. Plećaš 07/08/2002, 33, n.spec. =1); Povlen Mt.: Debelo Brdo hill (leg. anonim. 07/04/2001, 33, n.spec. = 1); (leg. M. Belanović 30/08/1982, 33, n.spec. = 1); Valjevo: Osečenica (v.), forest Bunčevica (leg. G. Džukić 29/05/1980, 33, n.spec. =2); Petnica (v.) (leg. $R$. Matić 22/05/1986, 33, n.spec. = 1); Vladimirci: Debrc (v.), Vlasenica (v.) (leg. M. Belanović 20/06/1976, 880, n.spec. $=1 ;$ 27/06/1976, 880); (leg. anonim. 01/03/1981, 880, n.spec. =1); South-eastern Serbia Besna Kobila Mt.: Kriva Feja (v.), Nesvrta (v.) (leg. G. Džukić, M. Kalezić, 20/06/2009, 30, n.spec. = 1); Bosilegrad: Brankovci (v.), toward v. Bistar (leg. G. Džukić 19/05/1978, 30, n.spec. =1); Southern Serbia Kukavica Mt.: Vučje, bellow Skobaljića Kula tower (leg. V. Vasić, 20/05/1977, 30, n.spec. =1); Medveđa: Tulare (v.), Jablanica (r.), $1.5 \mathrm{~km}$ from the village (leg. G. Džukić 17/05/1977, 30, n.spec. = 1); Srem Fruška Gora Mt.: Čerević (v.), Andrevlje (leg. anonim., 11.05.-22.05.1975, 777, n.spec. = 16); Čortanovci (v.) (leg. G. Džukić 31/05/1978, 156, n.spec. = 13); Ležimir (leg. anonim. 20.05.-25.05.1982, 904, n.spec. $=2$ ); mountain range, near crossroad to Monastery Grgeteg (leg. G. Džukić 09/07/1980, 904, n.spec. =1); Osovlje (leg. G. Džukić 20.05.-25.05.1979, 126, n.spec. = 5); Zmajevac (leg. Z. Dunđerski 28/05/1983, 904, n.spec. = 1); (leg. anonim. 23/05/1965, 904, n.spec. =2; May 1975, 904); (leg. Z. Dunđerski 1980, 904, n.spec. =1); Ruma: Grabovci (v.), Crepovac (leg. M. Stanković 17/08/1996, 29, n.spec. = 1); Grabovačko-Vitojevačko island, Crepovac (leg. M. Stanković 19/05/1996, 29, n.spec. = 1); Nikinci (v.), boder between forests Pavlaka and Baradinci (leg. M. Stanković 11/04/1996, 29, n.spec. = 2); Platičevo (v.), Novi Vitojevci, forest Baradinci, Vučkovac (leg. M. Stanković 21/05/1996, 29, n.spec. = 1); Šumadija Avala Mt.: Čarapićev Brest (leg. G. Džukić, 01/06/2002, 36, n.spec. =1); Šuplja Stena, near old mine (leg. T. Vukov, N. Tomašević Kolarov, M. Slijepčević, A. Urošević 30/10/2013, 36, n.spec. = 1); Trešnja (leg. anonim. 21/06/1990, 36, n.spec. = 1); (leg. G. Džukić 12/04/1983, 36, n.spec. =1); hothouse (leg. anonim. 26/06/1989, 36, n.spec. = 1); (leg. G. Džukić, M. Brajković 01/06/1980, 36, n.spec. = 2); (leg. M. Brajković 18/03/1981, 36, n.spec. = 1); Beograd: Čukarica, Košutnjak (leg. anonim. 09/05/1969, 35, n.spec. $=3$; 22/04/1970, 35); Palilula, Karaburna, Patris Lumumba street (leg. G. Džukić 06/10/1986, 35, n.spec. = 1); Umka, Lipik (leg. M. Milenković s. d., 35, n.spec. =1); Voždovac, Braće Jerković, central cemetery (leg. anonim. 26/04/1985, 35, n.spec. $=2$ ); Zvezdara, Kaluđerica (leg. S. Denčić 23/08/1994, 35, n.spec. = 1); Bukulja Mt. (leg. anonim. 16/04/1950, 34, n.spec. = 1); Jagodina - Kragujevac: Slatina (v.) (leg. I. Ham 24/09/1978, 903, n.spec. = 16); Kosmaj Mt.: Koraćica (v.), toward the village (leg. G. Džukić, M. Kalezić 25/07/1999, 34, n.spec. =1); (leg. anonim. 22/08/1954, 34, n.spec. = 1); Rudnik Mt.: eastern slopes (leg. I. Ham 27/06/1978, 34, n.spec. =1); Sopot: Rogača (v.) (leg. A. Ivanović 27/05/2007, 34, n.spec. =1); Western Serbia Čačak: city, centre (leg. Lj. Tomović, R. Ajtić, 01/05/2002, 149, n.spec. =1); Kraljevo: Savovo (v.), Studenica (r.) (leg. V. Stevanović 13/07/1977, 149, n.spec. = 1); Požega: Zdravčići (v.) (leg. anonim. s. d., 149, n.spec. = 1); Tara planina Mt.: Batura, Đurđevo brdo hill (leg. G. Džukić 11/06/1969, 149, n.spec. = 2); Mitrovac, new road toward Mitrovac (leg. G. Nonveiller 23/05/1984, 149, n.spec. = 2); Slovenia Idrija Idrijske Krnice (v.): Bočh. (leg. J. Gregori, 06/07/1975, 37, n.spec. = 1); Julian Alps Krn: Krnsko jezero Lake (leg. B. Kryštufek, 04/07/1975, 905, n.spec. =1); Koper Dobrava: Belveder (leg. B. Kryštufek, 16/09/2005, 37, n.spec. = 3); (leg. B. Kryštufek 10/07/1975, 905, n.spec. $=$ 3); Ljubljana Ig (v.): Kremenica (v.) (leg. B. Petrov, 01/08/1973, 37, n.spec. =1); (leg. G. Džukić 27/04/1979, 37, n.spec. = 2); Notranjsko Ribnica: Travna Gora (leg. B. Kryštufek, June 1975, 905, n.spec. $=2$ ).

\section{Pseudopus apodus}

Bosnia \& Herzegovina Herzegovina Čapljina (leg. S. Marinković, June 1985, 198, n.spec. = 1); Macedonia Vardar Zone Demir Kapija (leg. anonim., 14/04/1972, 177, n.spec. = 1); Montenegro Mediterranean Montenegro Boka Kotorska: Morinj (v.), Morinjska Reka

(r.), waterfall (leg. G. Džukić, M. Kalezić, 13/10/1996, 199, n.spec. =1); Budva: city (leg. Brkić 01/12/1997, 199, n.spec. = 1); Sveti Stefan, Đenaši (leg. R. Anđus May 1995, 199, n.spec. =1); Kotor: Dobrota (leg. V. Kalafatić 04/05/1977, 199, n.spec. $=1$ ); Submediterranean Montenegro Danilovgrad: Garač Mt., Donji Garač (leg. G. Džukić, M. Kalezić, 25/04/2002, 178, n.spec. =1); 
Rumija Mt.: Godinje (v.), road toward the village (leg. J. Crnobrnja, I. Aleksić 30/05/1996, 178, n.spec. =1); Skadarsko jezero Lake: Donje Selo (v.), Poseljanih. (leg. G. Džukić 11/10/1996, 178, n.spec. =1); Orahovačko Polje (leg. G. Džukić 09/06/1980, 178, n.spec. =1).

\section{CHAMAELEONIDAE}

\section{Furcifer pardalis}

Mauritius (leg. T. Anđić October 1997, 155, n.spec. =1).

\section{GEKKONIDAE}

\section{Hemidactylus turcicus}

Croatia Central Dalmatia Biševo (i.) (leg. anonim., 20/04/1949, 166, n.spec. = 4); Korčula (i.): Lumbarda (leg. B. Kral 20.06.-02.07.1969, 188, n.spec. = 1; 23.07.-02.08.1969, 188); Lastovo (i.) (leg. anonim. 22/04/1949, 143, n.spec. = 1); Split: Institute for Marine Biology (leg. anonim. 29/07/1948, 188, n.spec. = 2); Vis (i.): Mali Paržanj (i.) (leg. anonim. 10/05/1950, 143, n.spec. =2); Southern Dalmatia Dubrovnik: Lokrum (i.) (leg. P. Petrović, 16/05/1972, 143, n.spec. = 1); Montenegro Mediterranean Montenegro Boka Kotorska: Lepetane (leg. G. Džukić, 27/07/1972, 127, n.spec. = 1); Luštica Peninsula, Đuraševići, beach, Krtole (v.) (leg. V. Stevanović August 2010, 127, n.spec. =7); Gošići (v.), between Krašići (v.) and Radovići (v.) (leg. anonim. 27/08/1994, 127, n.spec. = 1); Krtole (leg. V. Stevanović 08/12/2008, 127, n.spec. = 1); Budva: Petrovac, Reževići (v.) (leg. D. Kataranovski 08/10/2004, 187, n.spec. =1); Sutomore (leg. O. Ivković 09/09/1978, 187, n.spec. = 1); Sveti Stefan (leg. A. Hegediš 27/09/1995, 187, n.spec. =1); Herceg Novi: Topla (leg. D. Grubišić 15/09/2008, 187, n.spec. =1).

\section{Mediodactylus kotschyi}

S. I. (leg. anonim. s. d., 1592, n.spec. =7); Bulgaria Struma (r.) - valley Kresna (r.) gorge: Kresnansko Hanče (leg. Lj. Tomović, 17/08/2002, 134, n.spec. =9); Greece Chalkidiki Stavros: Olimpijada (v.) (leg. S. Marinković, 06.07. \& 10.07.2003, 133, n.spec. = 2); i. Crete Heraklion (leg. S. Marinković, 27/08/1988, 133, n.spec. =2); Iraq Bagdad(leg. S. Marinković, 1983 \& 1984, 132, n.spec. = 2); Macedonia Vardar Zone Negotino: city (leg. G. Džukić, M. P., 27/05/2004, 131, n.spec. = 4); Pepelište (v.) (leg. G. Džukić, M. P. 26/05/2004, 131, n.spec. = 21); Skoplje: Mičurin (leg. anonim. 01.06.-12.06.2004, 130, n.spec. = 34); Taftalidže (leg. S. Petkovski May 2001, 130, n.spec. = 1); Star Dojran: city (leg. G. Džukić, M. P. 26/05/2004, 129, n.spec. = 27); Štip (leg. anonim. s. d., 131, n.spec. $=2$ ); Veles: city (leg. anonim. 19/07/2004, 128, n.spec. $=27$ ); Karaslari (v.), motel "Makedonija" (leg. S. Petkovski 01/07/2001, 128, n.spec. =3); Kojnik (leg. G. Džukić 11/05/1972, 128, n.spec. =1; 14/05/1980, 128); Serbia Metohia Prizren: city (leg. R. Ajtić, 06/10/1998, 135, n.spec. =6); Šmadija Beograd: Savski Venac, Pajsijeva street (leg. A. Cetković, 23/05/2009, 135, n.spec. $=1$ ).

\section{Tenuidactylus fedtschenkoi}

Afghanistan Farjab Majmana (leg. anonim., 13/08/1972, 795, n.spec. = 4); Samangan Takht-e-Rostam (leg. anonim., 16/08/1972, 795, n.spec. =6).

\section{LACERTIDAE}

\section{Algyroides nigropunctatus}

Croatia Istria Rovinj: Šorići (v.) (leg. G. Džukić, 11/04/1969, 90, n.spec. =11); Kvarner Cres (i.): Cres (leg. N. Tvrtković, 07/04/1976, 101, n.spec. = 1); Merag port (leg. anonim. 16/04/1964, 101, n.spec. =1; 22/09/1964, 101); Krk (i.) (leg. D. Jelić s. d., 101, n.spec. =14); (leg. Radovanović 
March 1938, 101, n.spec. = 1); Northern Dalmatia Crikvenica: Kačjak (v.) (leg. J. Purger, 23/04/1989, 90, n.spec. = 2); Macedonia Central Mountains Treska (r.): Matka gorge (leg. $P$. Jakšić, 27/04/1992, 85, n.spec. =1); South-western Region Ohridsko jezero Lake: Peštani (v.) Trpejca (v.) (leg. anonim., 25/08/1969, 85, n.spec. =2); (leg. G. Džukić 18/09/1978, 85, n.spec. =1); Prespansko jezero Lake: Oteševo (v.) (leg. anonim. 18/05/1980, 85, n.spec. =1); (leg. G. Džukić 19/09/1978, 85, n.spec. =4); Stenje (v.), Ćošak (leg. G. Džukić 25.05. \& 29.05.2008, 85, n.spec. =12); Montenegro Central Montenegro Morača (r.) - canyon: Babiće (v.) (leg. R. Ajtić, 29/07/2001, 86, n.spec. = 1); Mrtvica (r.) - canyon (leg. R. Ajtić 27/07/2001, 86, n.spec. =1); Mediterranean Montenegro Boka Kotorska: Gornji Stoliv (leg. A. Ćetković, 26/05/1996, 86, n.spec. = 1); Luštica Peninsula, Đuraševići, beach, Krtole (v.) (leg. V. Stevanović August 2010, 86, n.spec. = 1); Radovići (v.) (leg. J. Crnobrnja 20/09/1986, 86, n.spec. =1); Tivat: Lastva Grbaljska, Gornja Lastva (leg. anonim. 26/05/1996, 86, n.spec. $=2$ ); Submediterranean Montenegro Bar: Limljani (v.), lower part (leg. G. Džukić, M. Kalezić, M. Marković, 31/05/2003, 84, n.spec. = 1); Podgorica: Tuzi (v.), Dečić, foothill (leg. I. Aleksić 29/05/1996, 84, n.spec. =1); Skadarsko jezero Lake: Bisage (i.) (leg. anonim. 20/08/1990, 84, n.spec. =1); (leg. G. Džukić, M. Kalezić, D. Bejaković, I. Aleksić, J. Crnobrnja Isailović 16/05/1994, 84, n.spec. = 37); (leg. G. Džukić, M. Kalezić, J. Crnobrnja, I. Aleksić 31/05/1996, 84, n.spec. = 5); Livari (v.), Briska, Maskodra (leg. G. Džukić, M. Kalezić 12/10/1996, 84, n.spec. = 1); Pinčići (v.) (leg. M. Kalezić, A. Ivanović, I. Aleksić 30/05/1995, 84, n.spec. $=1$ ); Tophana (i.) (leg. G. Džukić 07/06/1980, 84, n.spec. = 3); Serbia Metohia Prizren: Vrbnica (v.) (leg. G. Džukić, 06/07/1969, 175, n.spec. =2); Zjum (v.), Zjumsko jezero Lake (leg. G. Džukić 20/07/1980, 175, n.spec. = 1); Zrze (v.), Beli Drim (r.) - gorge, Švanski most (leg. V. Stevanović 09/07/1977, 175, n.spec. =4); Prokletije Mts.: Beli Drim (r.) - spring, Radavačka pećina cave (leg. G. Džukić 07/06/1977, 175, n.spec. = 1); Slovenia Koper Vanganelsko jezero Lake (leg. BID "Josif Pančić", 26/08/1976, 123, n.spec. =1).

\section{Dalmatolacerta oxycephala}

S. I. (leg. anonim. s. d., 1594, n.spec. = 17); Bosnia \& Herzegovina Herzegovina Nevesinje: Trusina (v.), Berkovići (v.) - Nevesinje, road (leg. G. Džukić, 15/05/1983, 805, n.spec. = 1); Croatia Central Dalmatia Vis (i.): Mali Paržanj (i.) (leg. anonim., 10/05/1950, 791, n.spec. $=$ 1); (leg. anonim. 06/05/1950, 791, n.spec. =4); Southern Dalmatia Mljet (i.): Korita (leg. V. Majić, 02/05/1990, 791, n.spec. = 1); Malo jezero Lake (leg. V. Majić 04/05/1990, 791, n.spec. = 1); Saplunara (leg. V. Majić 02/05/1990, 791, n.spec. = 1); Švicarski put (leg. V. Majić 03/05/1990, 791, n.spec. =1); Montenegro Central Montenegro Danilovgrad: Garač Mt., Vodine (leg. G. Džukić, M. Kalezić, 25/04/2002, 147, n.spec. = 2); Prekornica Mt., Acrdani, Dolovi (leg. G. Džukić, M. Kalezić, M. Marković s. d., 147, n.spec. =1); Mediterranean Montenegro Bar: Đuravci (v.), beach (leg. anonim., 16/07/1992, 827, n.spec. = 1); Boka Kotorska: Morinj (v.), Morinjska Reka (r.), waterfall (leg. G. Džukić, M. Kalezić 13/10/1996, 827, n.spec. =9); Perast (leg. M. Kalezić 13/10/1996, 827, n.spec. = 1); Budva: Petrovac, Katići (i.) (leg. anonim. 26/07/1959, 827, n.spec. =6); Northern Montenegro Tara (r.) - canyon: Tepca (v.) (leg. R. Ajtić, 12/07/2001, 147, n.spec. = 3); Submediterranean Montenegro Cetinje (Ćeklići): Kućišta (v.), Crno Korito (leg. G. Džukić, M. Kalezić, M. Marković, 20/06/2004, 876, n.spec. = 2); Vučji Do (v.) (leg. G. Džukić, M. Kalezić, M. Marković 26/06/2004, 876, n.spec. = 1); Lovćen Mt.: Bjeloši (v.), Država (leg. G. Džukić, M. Kalezić, J. Crnobrnja, I. Aleksić 27/05/1996, 876, n.spec. = 2); Lašor (leg. G. Džukić, M. Kalezić, J. Crnobrnja, I. Aleksić 27/05/1996, 876, n.spec. =14); (leg. M. Kalezić, A. Ivanović, I. Aleksić 26/05/1995, 876, n.spec. = 1; 876); Orjen Mt.: Kamenica (v.), Borići (leg. G. Džukić, M. Kalezić 25/08/2001, 881, n.spec. = 3); Kruševice (v.), Lokanj (leg. G. Džukić, M. Kalezić 11/10/1996, 881, n.spec. =4); Ubli (v.), school (leg. G. Džukić, M. Kalezić 25/08/2001, 881, n.spec. =2); Skadarsko jezero Lake: Beška (i.) (leg. anonim. 05/06/1990, 890, n.spec. = 23); (leg. G. Džukić, M. Kalezić, D. Bejaković, I. Aleksić, J. Crnobrnja, E. Kletečki, N. De Luca 06/06/1990, 919, n.spec. = 13; 05.06.-07.06.1990, 919); Dužica (i.) (leg. anonim. s. d., 896, n.spec. =17); Golubovo (i.) (leg. anonim. 05/06/1990, 886, n.spec. =79; April 1992, 889; June 1990, 889); (leg. G. Džukić, M. Kalezić, D. Bejaković, I. Aleksić, J. Crnobrnja, E. Kletečki, N. De Luca 05.06. \& 06.06.1990, 889, n.spec. $=2$ ); Gorica I (i.), Radevac (leg. anonim. 10/05/1992, 893, n.spec. =7); Gorica II (i.) (leg. anonim. 16/08/1990, 894, n.spec. =13); Gorica III (i.) (leg. anonim. 16/08/1990, 
891 , n.spec. $=8 ; 08 / 05 / 1992,808 ; 09 / 05 / 1992,910 ; 1537 ; 10 / 05 / 1992,891 ; 12 / 10 / 1992$, 917; s. d., 918); (leg. J. Crnobrnja 12/10/1992, 891, n.spec. = 4); Gorica Kranja (i.) (leg. anonim. 07/06/1990, 909, n.spec. = 30); Gorica Smojs (i.) (leg. anonim. 07/06/1990, 908, n.spec. =60; 09/05/1992, 913; May 1992, 913); Krnjice (leg. G. Džukić 04/12/1992, 914, n.spec. =1); Moračnik (i.) (leg. anonim. 07/06/1990, 803, n.spec. =6); Planik (i.) (leg. anonim. 17/08/1986, 906, n.spec. = 14; August 1990, 802; June 1990, 901); Starčevo (i.) (leg. anonim. August 1990, 803, n.spec. =6); (leg. G. Džukić August 1990, 803, n.spec. =2); Starčevo (i.) - Moračnik (leg. G. Džukić, M. Kalezić, D. Bejaković, I. Aleksić, J. Crnobrnja, E. Kletečki, N. De Luca 04.06.-08.06.1990, 803, n.spec. = 1); Široka Gorica (i.) (leg. G. Džukić, M. Kalezić, D. Bejaković, I. Aleksić, J. Crnobrnja Isailović 16/05/1994, 907, n.spec. = 8); Vranica (leg. G. Džukić, M. Kalezić, D. Bejaković, I. Aleksić, J. Crnobrnja Isailović 16/05/1994, 897, n.spec. $=26$ ); Zalug (i.) (leg. anonim. 09/05/1992, 804, n.spec. $=3$; 10/05/1992, 804; 15.07. \& 16.07.1992, 804); (leg. anonim. 07/06/1990, 883, n.spec. $=24$; s. d., 884; 914); Western Montenegro Nikšić: Koprivice (v.), Čista Vlaka (leg. anonim., 11/06/2005, 122, n.spec. =5); Vilusi (v.), Donja Spila, Voluje oko (leg. G. Džukić, M. Kalezić, M. Marković 25/06/2004, 122, n.spec. =1); Orjen Mt.: Grahovo, dam (leg. G. Džukić, M. Kalezić, J. Crnobrnja, I. Aleksić 25/05/1996, 122, n.spec. =15).

\section{Darevskia praticola}

Russia Krasnodarskij Region Krepostnaja (v.) (leg. S. Litvinchuk, 17/06/2002, 46, n.spec. = 2); Republic Chechenia Groznenskaja Region: okresnosti st. Šelkovskoj (leg. leg. I.S. Darevski, det. N.V. Šibanov, June 1951, 46, n.spec. =1); Serbia Banat Vršački breg Mt.: Široko Bilo (leg. G. Džukić, 09/06/1970, 100, n.spec. = 7; 10/06/1970, 100); (leg. A. Ćetković 05/05/1987, 100, n.spec. = 1); (leg. anonim. 23/04/1992, 100, n.spec. = 4); (leg. G. Džukić, M. Kalezić 07/05/2004, 164, n.spec. = 7); (leg. G. Džukić, M. Kalezić, K. Ljubisavljević, A. Ivanović 09.06. \& 26.06.2002, 50, n.spec. = 23); (leg. G. Džukić, M. Kalezić, K. Ljubisavljević, Lj. Tomović 19.05.2003, 31.05.2003, 11.06.-12.06.2003, 27.06.2003, 49, n.spec. = 23); (leg. K. Ljubisavljević, A. Ivanović, M. Kalezić, T. Vukov 04/06/2009, 165, n.spec. = 10); (leg. leg majki: K. Ljubisavljević, T. Vukov, G. Džukić, A. Ivanović, M. Kalezić born in July \& August 2009, 92, n.spec. =17); Central Serbia Kopaonik Mt.: Jošanička Banja (leg. J. Crnobrnja Isailović, 02/06/1986, 47, n.spec. = 1); Savinac (leg. J. Crnobrnja Isailović 24/07/1987, 47, n.spec. = 4); North-eastern Serbia Đerdapska klisura gorge: Cuce (leg. anonim., 23/07/1978, 51, n.spec. =1); Kladovo: Petrovo Selo (v.), Tekija (leg. anonim. 20/05/1948, 51, n.spec. =1); Velesnica (v.) (leg. J. Crnobrnja Isailović, I. Aleksić 29/05/1998, 51, n.spec. = 8); Negotin: Brza Palanka (v.), Monastery Vratna (leg. V. Vasić 30/04/1972, 51, n.spec. = 2); Zamna (r.) - gorge (leg. G. Džukić 21/04/1988, 51, n.spec. $=1$ ); Šumadija Avala Mt.: Čarapićev Brest, Beli Potok (v.), near the village (leg. A. Ćetković, J. Čupić, 10.04. \& 19.04.1993, 48, n.spec. = 2); (leg. G. Džukić, M. Kalezić, K. Ljubisavljević 01/06/2002, 48, n.spec. = 3); Trešnja (leg. anonim. 02.05.1992, 22.04 .1993 and June 1990, 52, n.spec. $=10 ; 21.05 .1990,21.06 .1990$ and June 1990, 52); (leg. anonim. 22.05.1992, 10.04.1993, 22.04.1993, 53 , n.spec. $=22$ ); (leg. K. Ljubisavljević 02/05/2003, 48, n.spec. $=1$ ); Beograd: Čukarica, Košutnjak (leg. G. Džukić 12/10/1971, 48, n.spec. =1).

\section{Dinarolacerta montenegrina}

Montenegro Eastern Montenegro Prokletije Mts.: Đebeza Mt. (leg. G. Džukić, M. Kalezić, 03/06/2005, 76, n.spec. =4; 77); (leg. G. Džukić, M. Kalezić, A. Ivanović, I. Aleksić 31.05. \& 02.06.1996, 76, n.spec. $=3$ ).

\section{Dinarolacerta mosorensis}

Bosnia \& Herzegovina Herzegovina Gacko: Bjelašnica Mt., Mangrop (leg. G. Džukić, 05/08/1970, 184, n.spec. = 2); Croatia Central Dalmatia Biokovo Mt.: Vošac - Sveti Jure (leg. G. Džukić, 22.07. \& 23.07.1969, 81, n.spec. = 5); Montenegro Central Montenegro Danilovgrad: Prekornica Mt., Ponikvice (leg. G. Džukić, M. Kalezić, 10/06/1997, 79, n.spec. =1); Ponikvice, $3 \mathrm{~km}$ from Ponikvica toward Župa (leg. G. Džukić, M. Kalezić 23/08/2001, 79, n.spec. =7); (leg. G. Džukić, M. Kalezić, M. Marković 19/06/2004, 79, n.spec. = 10); Maganik Mt.: Štitovo, plateau 
(leg. V. Vasić 07/07/1983, 79, n.spec. = 1); Mrtvica (r.) - canyon (leg. R. Ajtić 27/07/2001, 79, n.spec. = 2); Northern Montenegro Durmitor Mt.: Golubinje, bellow (leg. G. Džukić, 03/08/1985, 80, n.spec. =1); Valovito jezero Lake (leg. anonim. s. d., 80, n.spec. =1); (leg. G. Džukić 11/07/1983, 80, n.spec. =3); Virak (leg. M. Raković 10/08/2004, 80, n.spec. =1); Zminje jezero Lake (leg. BID "Josif Pančić" 10/07/1979, 78, n.spec. =15); (leg. Z. Franolić 29/08/1984, 80, n.spec. =4); (leg. anonim. 03/08/1980, 80, n.spec. $=1$; s. d., 80); Submediterranean Montenegro Lovćen Mt.: Međuvršje, between the lake and Međuvršje (leg. G. Džukić, 28/07/1988, 82, n.spec. =2); (leg. M. Kalezić, A. Ivanović, I. Aleksić 27/05/1995, 82, n.spec. $=9$ ).

\section{Eremias sp.}

Afghanistan Kabul Guldara (leg. anonim., 21/08/1972, 167, n.spec. =1).

\section{Iberolacerta horvathi}

Croatia Gorski Kotar Delnice: Crni Lug (v.), Markov Brlog (leg. R. Rudsner, 15/07/1964, 189, n.spec. = 3); Markov Brlog, bellow Smrekovac (leg. anonim. 14/07/1964, 189, n.spec. = 1); Lika Velebit Mt.: Jablanac (v.), Mirovo (leg. B. Jalžić, 16/08/1976, 189, n.spec. =1); (leg. M. Radovanović 03/08/1954, 189, n.spec. = 1); Slovenia Julian Alps Mangart Mt.: foothill (leg. B. Kryštufek, S. Brelih, Summer 1965, 170, n.spec. =6); Triglav Mt.: Crno jezero Lake (leg. S. Brelih 23/09/1955, 170 , n.spec. $=1)$.

\section{Lacerta agilis}

S. I. (leg. anonim. s. d., 1590 , n.spec. $=21$ ); Bosnia \& Herzegovina Bosnia Bosanski Petrovac: 12-15 km toward Jajce (leg. V. Stevanović, 24/08/1983, 751, n.spec. = 1); Bosansko Grahovo: Lemići (v.), Lemića Gaj (leg. J. Šoti 30/04/1980, 751, n.spec. = 1); Cincar Mt.: Gornji Malovan (v.) (leg. G. Džukić 05/06/1985, 104, n.spec. = 4); Han Pijesak (leg. BID "Josif Pančić" 14/08/1976, 104, n.spec. = 1); Igman Mt.: Veliko Polje (leg. G. Džukić 01/08/1974, 751, n.spec. =4); Livanjsko Polje: Čelebić (v.) (leg. G. Džukić 18/05/1983, 751, n.spec. =1); Herzegovina Čvrsnica Mt.: Blidinje jezero Lake, Svinjača (leg. G. Džukić, 20/06/1971, 110, n.spec. = 2); Gacko: Bjelašnica Mt., Mangrop (leg. G. Džukić 05/08/1970, 110, n.spec. =1); Gacko Polje: Vrbica mine (leg. G. Džukić 15/06/1975, 110, n.spec. =1); Nevesinjsko Polje: Sopilje (v.), Dušila (leg. G. Džukić 16/05/1983, 110, n.spec. = 1); Croatia Baranja Tikveš (leg. J. Mikuška, 26/08/1970, 773, n.spec. = 1); Lika Gospić: Bužim (v.) (leg. G. Džukić, 02/06/1985, 773, n.spec. = 1); Plitvička jezera Lakes: Crna Rijeka (r.) (leg. N. Tvrtković 25/05/1977, 773, n.spec. = 2); Čorkova Uvala (leg. N. Tvrtković 25/05/1977, 773, n.spec. = 1); Podravina Donji Miholjac: Šemer (v.) (leg. D. Kovačić, 04/08/1977, 773, n.spec. = 3); Zagreb Maksimir (leg. Z. Krisković, Spring 1963, 773, n.spec. = 2); Vrhomac: Crni Vrh, road toward Kojane (v.) (leg. M. Radovanović 19/07/1961, 773, n.spec. = 1); Macedonia South-western Region Pelister Mt.: Golemo ezero Lake (leg. B. Petrov, July 1972, 882, n.spec. = 1); Široka (leg. G. Džukić 22/07/1979, 882, n.spec. = 1); Western Mountains Korab Mt.: sheepfold (leg. G. Džukić, 06/09/2010, 882, n.spec. = 1); Montenegro Central Montenegro Danilovgrad: Prekornica Mt., Srednja Ponikva (leg. G. Džukić, M. Kalezić, 10/06/1997, 107, n.spec. = 1); (leg. G. Džukić 19/06/2004, 107, n.spec. = 1); Kolašin: Mioska (v.), Kljajića livada (leg. G. Džukić, J. Crnobrnja Isailović, I. Aleksić 30/07/1997, 107, n.spec. = 1); Moračke planine Mts.: Lukavica Mt., Manito jezero Lake (leg. G. Džukić 23/08/2002, 107, n.spec. = 1); Eastern Montenegro Bjelasica Mt.: Bendovac (leg. G. Džukić, M. Kalezić, 23/08/1994, 142, n.spec. = 1); Katun Goleš (leg. G. Džukić, J. Crnobrnja, I. Aleksić 24/07/1997, 142, n.spec. = 1); Lalevića Dolovi (leg. anonim. August 2005, 142, n.spec. = 18); (leg. J. Crnobrnja Isailović 11/07/1996, 142, n.spec. =6); Prokletije Mts. (leg. S. Đorđević 09/07/2004, 142, n.spec. =1); Northern Montenegro Durmitor Mt.: Bosača (leg. BID “Josif Pančić", 12/07/1979, 98, n.spec. = 2); Bosačica (leg. BID “Josif Pančić" 07/07/1979, 98, n.spec. = 1); Ćurovac (leg. G. Džukić 24/06/1985, 752, n.spec. = 2); Donja Bukovica (v.) - Slatina (v.) (leg. G. Džukić 06/07/1986, 98, n.spec. =1); Komarnica (r.) - canyon, Klještina (leg. P. Jakšić 24/07/1985, 98, n.spec. = 1); Stožine (leg. P. Jakšić 24/07/1985, 98, n.spec. = 
1); Ninkovići (v.) (leg. G. Džukić 02/07/1986, 98, n.spec. =1); Pitomine (leg. A. Cetković 14/09/1991, 752 , n.spec. = 1); Ražana Glava (leg. Z. Franolić 24/07/1985, 98, n.spec. =1); Virak (leg. P. Jakšić 16/07/1987, 752, n.spec. =6); Zminičko jezero Lake (leg. anonim. 05/08/1959, 752, n.spec. $=1$ ); (leg. G. Džukić, M. Kalezić June 1997, 752, n.spec. = 1); (leg. J. Purger 03/07/1989, 752, n.spec. = 1); Motički Gaj (leg. B. Božičić 06/07/1983, 752, n.spec. =1); (leg. anonim. 01/08/1980, 752, n.spec. =1; s. d., 752); Sinjajevina Mt.: Kučajevica, Šaranske šume (leg. V. Stevanović 16/05/1984, 98, n.spec. = 1); Zminičko jezero Lake (leg. BID "Josif Pančić" 12/07/1979, 98, n.spec. = 2); Serbia Bačka Kanjiža: Kapetanski rit (leg. G. Džukić, 01/06/1978, 778, n.spec. =1); Subotica: Horgoš (v.), Seleven (leg. G. Džukić, M. Kalezić 11/04/1998, 778, n.spec. = 4); Makova sedmica, Hrastovača, Cirkuzantska bara pond (leg. G. Džukić, M. Kalezić 08/07/1999, 778, n.spec. =1); Mali Pijac (v.) (leg. J. Mikuška 01/05/1963, 778, n.spec. = 2); surrounding (leg. Abrašić 1960, 778, n.spec. =1); Banat Bela Crkva: Karađorđev Lug, toward Nera (r.) (leg. M. Marković, s. d., 105, n.spec. =1); Lug Kralja Aleksandra, between Jaruga and Mrtva Nera (leg. G. Džukić, M. Marković 25/03/2006, 105, n.spec. = 1); Mrtva Nera (leg. G. Džukić, M. Kalezić 04/05/2003, 105, n.spec. =1); Nera (r.), beach (leg. G. Džukić, M. Kalezić 16/03/2011, 105, n.spec. =1); (leg. G. Džukić, M. Kalezić, A. Ivanović, T. Vukov, M. Marković 06/06/2009, 105, n.spec. =1); Vračev Gaj (v.), Nera (r.) (leg. G. Džukić, M. Kalezić, M. Marković 04/05/2014, 105, n.spec. =2); Nera, enbankment (r.) (leg. G. Džukić 07/03/2014, 105, n.spec. $=1$ ); city lake (leg. G. Džukić 22/03/1970, 105, n.spec. =1); Beograd: Palilula, Krnjača, $3 \mathrm{~km}$ on enbankment toward Pančevo (leg. G. Džukić, M. Kalezić 13/04/2000, 106, n.spec. = 2); Pančevo bridge, canal after (leg. anonim. 29/03/2000, 106, n.spec. = 1); Reva pond (leg. A. Urošević, $M$. Anđelković 04/03/2016, 106, n.spec. = 2); (leg. leg. majki: M. Anđelković, A. Urošević s. d., 1576, n.spec. = 11); (leg. M. Anđelković, A. Urošević April-June 2017, 1575, n.spec. $=8$ ); Čoka: $3 \mathrm{~km}$ before the city (leg. G. Džukić 20/04/1982, 186, n.spec. = 4); Deliblatska peščara: Devojački Breg (leg. D. Pemac 18/10/2000, 106, n.spec. = 1); Jasenovo (v.), Jasenovačka šuma (leg. G. Džukić, M. Kalezić 22/08/1998, 106, n.spec. = 3); Nera (r.) (leg. G. Džukić, M. Kalezić 29/05/2010, 106, n.spec. =1); Šumarak (v.), Majur bara pond (leg. G. Džukić 16/05/1976, 106, n.spec. = 1; 09/04/1981, 106); Kikinda: Bašaid (v.) (leg. G. Džukić 21/04/1970, 186, n.spec. =1); Mokrin (v.) (leg. Lj. Tomović, R. Ajtić, Đ. Đoković April 2002, 186, n.spec. =7); Kovin: Crna bara pond (leg. G. Džukić 01.05. \& 02.05.1969, 111, n.spec. = 14); Kovin brigde, Banat side (leg. A. Urošević 06/07/2010, 111, n.spec. $=$ 1; 12/06/2011, 111); Nova Crnja: Radojevo (v.) (leg. anonim. 27/07/1993, 186, n.spec. =1); Novi Bečej (leg. anonim. 17/08/1980, 186, n.spec. =1); Novi Kneževac: Banatsko Aranđelovo (v.), Rabe (v.) (leg. G. Džukić 08/04/1983, 186, n.spec. =1); Opovo: canal (leg. M. Kalezić, A. Ivanović 11/04/2005, 106, n.spec. = 1); Srpska Crnja (leg. Lj. Tomović, R. Ajtić, Đ. Đoković April 2002, 186, n.spec. = 2); Vršac: surrounding (leg. D. Trajan 1959, 106, n.spec. = 1); Žitište: Torda (v.) (leg. anonim. 22/05/1991, 186, n.spec. =1); Central Serbia Kopaonik Mt.: Šipačine - Bačište (leg. $P$. Jakšić, 19/07/1985, 759, n.spec. = 1); Trepča (leg. anonim. July 1993, 759, n.spec. =1); Treska (leg. A. Ćetković 07/08/1986, 759, n.spec. =1); (leg. anonim. 24/07/1987, 759, n.spec. = 3); (leg. M. Niketić 07/06/1985, 759, n.spec. $=1$ ); Eastern Serbia Stara planina Mt.: Babin Zub (leg. J. Crnobrnja Isailović, 24/07/1993, 108, n.spec. = 3); Babin zub (leg. P. Jakšić 11/07/1991, 108, n.spec. = 1); Ponor (leg. G. Džukić, M. Kalezić, D. Jović 07/03/2008, 108, n.spec. =1); Žarkova Čuka, Dojčino vrelo (leg. J. Crnobrnja Isailović 15.07. \& 16.07.1994, 108, n.spec. = 2); (leg. A. Ćetković July 1994, 108, n.spec. = 5); North-western Serbia Maljen Mt.: Divčibare, Crna Kamenica (leg. J. Crnobrnja Isailović, 25/07/1999, 140, n.spec. = 1); Crna Reka (r.), toward the river (leg. G. Mesaroš s. d., 140, n.spec. =1); Golubac (leg. S. Matvejev 27/04/1974, 140, n.spec. =1); Kraljev Sto (leg. J. Crnobrnja Isailović 29/07/1999, 140, n.spec. = 1); Ljuti Krš (leg. M. Kalezić 06/10/2002, 140, n.spec. = 1); (leg. J. Šoti June 1978, 140, n.spec. = 1); (leg. leg. majki: M. Anđelković, A. Urošević s. d., 1573, n.spec. = 30; 1574); (leg. M. Anđelković, A. Urošević June 2017, 1572, n.spec. = 10); (leg. M. Kalezić 13/03/1994, 140, n.spec. = 3); (leg. M. Plećaš 06/08/2002, 140, n.spec. = 2); (leg. S. Stamenković 26/05/1994, 140, n.spec. =1); Povlen Mt.: Debelo Brdo hill (leg. anonim. 07/04/2001, 136, n.spec. =16; s. d., 136); Suvobor Mt.: Ravna Gora, Nikolića Koliba (leg. M. Plećaš 24/04/2004, 140, n.spec. = 1); Potok Grad (leg. M. Plećaš 24/04/2004, 140, n.spec. = 1); Pomoravlje Veliko Gradište: Srebrno jezero Lake (leg. anonim., 23/04/1995, 766, n.spec. = 1); (leg. G. Džukić, M. Kalezić 15/04/1994, 766, n.spec. =1); South-eastern Serbia Dukat Mt.: Karamanica, Crna Reka (r.), spring (leg. Lj. Tomović, R. Ajtić, 04/05/2002, 109, n.spec. = 6); Vlasina Plateau: Vardenik Mt., Strešer peak (leg. G. Džukić 19/07/1978, 109, n.spec. =1); (leg. anonim. 21/04/2007, 109, n.spec. =1); South-western Serbia 
Golija Mt.: Okruglica (leg. G. Džukić, M. Kalezić, 13/06/2001, 119, n.spec. = 1); Pešter Plateau: Hajrečevica (leg. anonim. 29/04/2010, 119, n.spec. =1); Sjenica, airport (leg. G. Džukić, M. Kalezić 27/04/2010, 119, n.spec. = 2); Uvac (r.), Molitva (r.) - canyon (leg. J. Šoti May 1979, 119, n.spec. = 1); Zlatar Mt.: Božetići (v.), Amidžićih. (leg. J. Crnobrnja Isailović 29/08/1997, 119, n.spec. = 2); (leg. G. Džukić, M. Kalezić 15/10/1996, 119, n.spec. =1); Western Serbia Požega: Skrapež (r.) (leg. BID "Josif Pančić", 04/07/1983, 91, n.spec. = 1); Tara planina Mt.: Golubovac (leg. G. Džukić 09/06/1969, 794, n.spec. = 2); Kaluđerske Bare (leg. G. Džukić 10/03/1981, 794, n.spec. =1); (leg. G. Džukić, J. Šoti 20/07/1978, 794, n.spec. = 2); Ljuto Polje, Karajića bare (leg. anonim. 30/06/2008, 794, n.spec. = 3); (leg. G. Džukić, M. Kalezić 30/06/2008, 794, n.spec. = 1); Šljivovica (v.), Kaluđerske Bare (leg. G. Džukić 21/06/1973, 794, n.spec. = 1); (leg. A. Ćetković 25/05/1992, 794, n.spec. =1); (leg. anonim. 2006, 794, n.spec. = 1); (leg. G. Džukić, M. Kalezić 12.06. \& 13.06.2001, 794, n.spec. = 2); Zlatibor Mt.: Crni Vrh, southern slopes (leg. M. Gvozdić 24/04/1992, 91, n.spec. =1); Čigota, Smiljanski Zakos (leg. M. Gvozdić 25/04/1992, 91, n.spec. = 3); (leg. M. Gvozdić 30/07/1992, 91, n.spec. = 4); Partizanske Vode, northern parts (leg. M. Gvozdić 21/04/1992, 91, n.spec. =1); (leg. K. Ljubisavljević 22/08/2003, 91, n.spec. =2); Tetrebovac, Gaj (v.) (leg. M. Gvozdić 31/07/1992, 91, n.spec. =1); Veliki Rzav (r.) (leg. M. Gvozdić 01/05/1992, 91, n.spec. = 4); (leg. S. Matvejev 01/06/1965, 91, n.spec. =1).

\section{Lacerta trilineata}

S. I. (leg. anonim. s. d., 1593, n.spec. =4); Bosnia \& Herzegovina Herzegovina Nevesinje: Trusina (v.), $5 \mathrm{~km}$ toward Nevesinje (leg. G. Džukić, 15/05/1983, 806, n.spec. = 1); Croatia Lika Velebit Mt.: Jurjevo (v.) (leg. N. Tvrtković, 03/07/1976, 190, n.spec. = 1); Zagora Obrovac: Monastery Krupa (leg. G. Džukić, 01/06/1985, 190, n.spec. =1); Greece i. Crete Heraklion (leg. A. Ćetković, 20/10/1986, 124, n.spec. = 1; 21/04/1990, 124); Rethimno (leg. anonim. 11/05/1987, 124, n.spec. = 1); Larissa Tembi (leg. anonim., 15/05/1987, 124, n.spec. = 1); Larissa Sykourion(leg. G. Džukić, 09/11/1972, 124, n.spec. =1); Macedonia Vardar Zone Veles: Vršnik hill, Zlatna pešter (leg. G. Džukić, 06/11/1973, 174, n.spec. = 1); Montenegro Central Montenegro Donja Morača (leg. G. Džukić, 10/07/1969, 89, n.spec. = 1); Mediterranean Montenegro Boka Kotorska: Morinj (v.), Morinjska Reka (r.), waterfall (leg. G. Džukić, M. Kalezić, 13/10/1996, 87, n.spec. = 1); Zelenika, Sasovići (v.) (leg. M. Kalezić, A. Ivanović, I. Aleksić 29/05/1995, 87, n.spec. = 1); Budva: Seoce (v.) (leg. G. Džukić, M. Kalezić 04/06/2005, 87, n.spec. = 1); Submediterranean Montenegro Danilovgrad: Garač Mt., Vodine (leg. G. Džukić, M. Kalezić, 25/04/2002, 88, n.spec. = 1); Zagarač Mt., Gornji Zagarač (leg. G. Džukić, M. Kalezić 26/04/2002, 88, n.spec. =1); (leg. anonim. 24/05/1996, 88, n.spec. =1); Podgorica: Medun, Orljevo (leg. G. Džukić, $M$. Kalezić 24/04/2002, 88, n.spec. = 1); Piperi, Gornji Crnci (v.), $6 \mathrm{~km}$ from the village (leg. anonim. 09/06/1997, 89, n.spec. = 1); Tuzi (v.), Dečić (leg. G. Džukić, J. Crnobrnja Isailović, I. Aleksić 29/05/1996, 88, n.spec. = 1); Skadarsko jezero Lake: Đuravci (v.) - Gornji Murići (v.) (leg. M. Kalezić, A. Ivanović, I. Aleksić 30/05/1995, 89, n.spec. =1); beach (leg. anonim. 1992, 89, n.spec. =1).

\section{Lacerta viridis}

S. I. (leg. anonim. s. d., 1591, n.spec. = 10); Bosnia \& Herzegovina Bosnia Šekovići: Monastery Ribnica (leg. G. Džukić, 30/06/2003, 151, n.spec. =1); Šipovo: Janja (r.) canyon (leg. Lj. Tomović, R. Ajtić 03.08.-15.08.2001, 151, n.spec. =1); Herzegovina Konjic: Borci (v.), Boračko jezero Lake (leg. anonim., 14/07/1955, 151, n.spec. =1); Croatia Baranja Draž: Batina (v.), near the monument (leg. G. Džukić, 09/04/1972, 27, n.spec. =1); Lika Velebit Mt. (leg. S. Radaković, 1955, 27, n.spec. =1); Southern Dalmatia Dubrovnik: Cavtat (leg. G. Džukić, 17/07/1969, 27, n.spec. = 1); Macedonia Central Mountains Babuna Mt.: Derven (leg. anonim., 10/05/1972, 18, n.spec. =4); Eastern Mountains Kratovo: Stracin (v.), Karadag (leg. BIOECO, 19/05/2002, 2, n.spec. = 1); (leg. G. Džukić 25/05/2004, 2, n.spec. =4); Karadag, Mokro jezero Lake (leg. G. Džukić 25/05/2004, 2, n.spec. =1); Karadag, Ploče (leg. G. Džukić 25/05/2004, 2, n.spec. = 1); Ploče (leg. G. Džukić 25/05/2004, 2, n.spec. = 5); (leg. G. Džukić, S. Petkoviski 24/06/1995, 2, n.spec. = 2); South-western Region Pelister Mt.: Rotino, acumulations (leg. BIOECO, 24/05/2002, 18, n.spec. = 1); Vardar Zone Dojransko jezero Lake: Dojran (leg. G. Džukić, 
26/05/2004, 17, n.spec. = 5); (leg. leg majki: G. Džukić born in August 2004, 17, n.spec. = 7); (leg. $M$. Radovanović 21/05/1958, 17, n.spec. = 1); Nov Dojran (leg. G. Džukić, S. Petkoviski 09/05/1997, 17, n.spec. =1); Strumičko Polje: Hamzali (v.), Suvi Laki (leg. anonim. 30/05/1970, 17, n.spec. $=2$ ); Valandovo: Đopčeli (v.), near crossroad to the village (leg. G. Džukić 26/05/2004, 17, n.spec. =1); Montenegro Central Montenegro Danilovgrad: Prekornica Mt., Acrdani, Dolovi (leg. G. Džukić, M. Kalezić, M. Marković, 19/06/2004, 11, n.spec. = 1); Bukotica (leg. G. Džukić, M. Kalezić 10/06/1997, 11, n.spec. = 2); Ponikvice (leg. G. Džukić, M. Kalezić 10/06/1997, 11, n.spec. =1); Eastern Montenegro Bijelo Polje: Radovići (leg. N. Labus, 23/05/2005, 24, n.spec. $=1$ ); Prokuplje: Vusanje, watchtower (leg. Z. Franolić 02/07/1984, 24, n.spec. =1); Northern Montenegro Durmitor Mt.: Komarnica (v.), Nevidio, Žuta Greda (leg. G. Džukić, 22/08/1984, 114, n.spec. =1); (leg. anonim. s. d., 114, n.spec. =1); Tara (r.) - canyon: Tepca (v.), Devojački most (leg. G. Džukić 06/07/1986, 114, n.spec. = 2); (leg. Lj. Tomović, Đ. Đoković July 2001, 114, n.spec. = 4); Submediterranean Montenegro Cetinje (Ćeklići): Kućišta (v.), Crno Korito (leg. G. Džukić, M. Kalezić, M. Marković, 20/06/2004, 10, n.spec. = 1); Ržani Do (v.), Cape (leg. G. Džukić, M. Kalezić, M. Marković 20/06/2004, 10, n.spec. = 1); Uba (v.), Ubine (leg. G. Džukić, M. Kalezić, M. Marković 21/06/2004, 10, n.spec. = 3); Vučji Do (v.), Sonina (leg. G. Džukić, M. Kalezić, M. Marković 26/06/2004, 10, n.spec. =1); Lovćen Mt.: Bjeloši (v.), Država (leg. G. Džukić, M. Kalezić, J. Crnobrnja Isailović, I. Aleksić 27/05/1996, 3, n.spec. = 1); Ivanova Korita (leg. G. Džukić, M. Kalezić, M. Marković 22/06/2004, 3, n.spec. = 1); Lašor (leg. G. Džukić, M. Kalezić, J. Crnobrnja Isailović, I. Aleksić 27/05/1996, 3, n.spec. = 3); (leg. M. Kalezić, A. Ivanović, I. Aleksić 26/05/1995, 3, n.spec. = 5); Orjen Mt.: Vrbanje (v.), Pod Duge, Mali Štirovnik, bellow (leg. M. Belanović 23/05/1984, 3, n.spec. = 1); (leg. G. Džukić 15/06/1972, 3, n.spec. = 1); Rumija Mt.: Pinčići (v.) (leg. M. Kalezić, A. Ivanović, I. Aleksić 30/05/1995, 9, n.spec. = 3); Sutorman, bend (leg. M. Kalezić, A. Ivanović, I. Aleksić 29/05/1995, 9, n.spec. = 1); Skadarsko jezero Lake: Gornji Murići (v.), above (leg. M. Kalezić, A. Ivanović, I. Aleksić 30/05/1995, 9, n.spec. = 4); Virpazar, Orahovštica (leg. G. Džukić 17/07/1969, 9, n.spec. =1); Vranjina Peninsula (leg. G. Džukić 25/07/1975, 9, n.spec. = 1); beach (leg. anonim. 1992, 9, n.spec. =2); Serbia Bačka Subotica: Makova Sedmica, Hrastovača, Cirkuzantska bara pond (leg. G. Džukić, M. Kalezić, 08/07/1999, 12, n.spec. = 1); Hrastovača, Čavolj (leg. G. Džukić, M. Kalezić 31/07/1999, 12, n.spec. = 2); Banat Bela Crkva: Dupljaja (v.), Dupljaja - Grebenac (v.), after Surduk (v.) (leg. G. Džukić, C. Eggert, 22/04/2004, 21, n.spec. = 1); Stara Palanka (v.) (leg. G. Džukić, M. Kalezić 22/06/1994, 21, n.spec. = 7); Deliblatska peščara: Čardak (leg. BID "Josif Pančić" 13/05/1979, 1, n.spec. = 6); Deliblato (v.) (leg. G. Džukić 19/05/1969, 16, n.spec. = 1); Flamunda (leg. G. Džukić 08/05/1969, 16, n.spec. $=1 ; 11 / 04 / 1970,16$ ); (leg. V. Stevanović 01/05/1970, 16, n.spec. = 1); Kajtasovo (v.), Mali Pesak (leg. G. Džukić, M. Kalezić June 1999, 1, n.spec. =2); Mali Pesak, Kremzerov Salaš (leg. $M$. Marković s. d., 1 , n.spec. =1); Mali Pesak, Svinjska plaža (leg. anonim. 20/06/2008, 1, n.spec. = 2); (leg. G. Džukić, M. Kalezić, M. Marković June 2007, 1, n.spec. = 1); Šumarak (v.), Majur bara pond (leg. G. Džukić 16/05/1976, l, n.spec. =1; 09/04/1981, 1); (leg. G. Džukić, M. Kalezić 22/06/1994, 1, n.spec. = 1); (leg. G. Džukić 03/05/1969, 16, n.spec. = 4); Utrine, Stevanove ravnice, between Knjažev Salaš and Salaš Vlaških (leg. G. Džukić 10/06/2004, l, n.spec. = 1); Vladimirovac (v.), Devojački bunar (leg. anonim. 02/06/1973, 16, n.spec. =1); (leg. G. Džukić 08/05/1969, 16, n.spec. =1); Devojački bunar - Mala Tilva (leg. D. Pavićević 15/09/1977, 16, n.spec. =2); Volovska Paša (leg. V. Stevanović 07/07/1987, 16, n.spec. = 1); Đurica pond (leg. G. Džukić, M. Kalezić 21/05/1998, 1, n.spec. = 1); Hubertov Salaš (leg. anonim. 23/07/1996, 1 , n.spec. = 1); (leg. leg majki: K. Ljubisavljević, T. Vukov, G. Džukić, A. Ivanović, M. Kalezić, M. Cvijanović born in August \& September 2008 \& 2009, 19, n.spec. = 37); (leg. M. Čobanov 1969, 16, n.spec. = 1); (leg. M. Radovanović 17/06/1956, 16, n.spec. = 3); (leg. S. Matvejev 31/03/1954, 16, n.spec. =1); Kovin: Crna bara pond (leg. G. Džukić 02/05/1969, 21, n.spec. = 1); Vršac: Jablanka (v.) (leg. G. Džukić, M. Kalezić, M. Marković 19/10/2002, 13, n.spec. =2); Vršački breg Mt.: Kula (leg. G. Džukić 30/03/1983, 13, n.spec. =1); Malo Središte (v.), toward Gudurički vrh (leg. G. Džukić, M. Kalezić 19/05/2003, 13, n.spec. = 3); Markovac (v.) (leg. G. Džukić, M. Kalezić 09/06/2004, 13, n.spec. =1); mountain house (leg. G. Džukić 06/03/2009, 13, n.spec. = 2); Široko Bilo (leg. G. Džukić 19/06/1969, 13, n.spec. =2; 09/06/1970, 13; 17/05/1976, 13); (leg. anonim. 17/07/1959, 13, n.spec. =1;09/06/2002, 13); (leg. G. Džukić s. d., 13, n.spec. =2); (leg. G. Džukić, M. Kalezić 07/05/2004, 13, n.spec. =1); Central Serbia Kopaonik Mt. (leg. M. Radovanović, 30/05/1951, 20, n.spec. $=1$ ); Kuršumlija: Rudare (v.), Rudare (v.) - Prolom Banja, road (leg. Lj. Tomović 02/05/1998, 20, n.spec. = 1); (leg. Lj. Tomović, K. Ljubisavljević 20/06/1998, 20, n.spec. = 1); Leskovac (leg. N. Labus 15/04/2004, 20, n.spec. = 2); Prokuplje: Mala Draguša (v.) (leg. A. Ćetković 01/05/1986, 20, n.spec. = 1); $15 \mathrm{~km}$ to the west (leg. M. Radovanović 22/07/1961, 20, n.spec. $=1$ ); Raška: Baljevac (v.) (leg. J. Karamarković 12/06/2005, 20, n.spec. =2); Trstenik: Donja Crnišava 
(v.) (leg. K. Ljubisavljević 29.07.-31.07.1997, 20, n.spec. =4); Eastern Serbia Pirot: Krupac (v.), Krupačko jezero Lake, Brkina vrela (leg. G. Džukić, 23/05/1977, 14, n.spec. =2); Krupačko Vrelo (leg. G. Džukić 23/05/1977, 14, n.spec. = 1); Sićevačka klisura gorge: Crveni Breg (v.), Krst hill (leg. $B$. Žiljak 10/06/1997, 14, n.spec. = 1); Gradište (v.), Čurilovac (leg. Lj. Tomović, K. Ljubisavljević 20/06/1998, 14, n.spec. = 1); Ostrovica (v.), Banjica (leg. Lj. Tomović, K. Ljubisavljević 20/06/1998, 14, n.spec. = 1); Stara planina Mt.: Crni Vrh (leg. BID "Josif Pančić" 16/07/1983, 14, n.spec. = 2); (leg. BID "Josif Pančić” 17/07/1983, 14, n.spec. =1); Kosovo Gnjilane: Buzalak (v.), Dobri Čiča hill (leg. G. Džukić, 17/05/1977, 22, n.spec. = 2); Ibar (r.) - valley: Lozište (v.), Košutovački potok (leg. P. Jakšić 24/07/1987, 22, n.spec. = 1); Kosovo Polje: Obilić (v.), surrounding (leg. G. Pasuljević April 1967, 22, n.spec. = 1); Novo Brdo hill: Bostane (v.) (leg. P. Jakšić 15/05/1985, 5, n.spec. = 1); Čuljkovci (v.) (leg. P. Jakšić 05/05/1985, 5, n.spec. = 3); Klobukar (v.) (leg. P. Jakšić 15/05/1985, 5, n.spec. $=1 ; 22 / 05 / 1985,5 ; 10 / 04 / 1986,5)$; Manišince (v.) (leg. P. Jakšić 22/05/1985, 5, n.spec. = 1); Prekovce (v.) (leg. P. Jakšić 22/05/1985, 5, n.spec. $=1 ; 05 / 06 / 1985,5)$; Šumaci (v.) (leg. P. Jakšić 22/05/1985, 5, n.spec. = 1); Trnićevce (v.) (leg. P. Jakšić 10/04/1986, 5, n.spec. = 1; 27/05/1986, 5); (leg. P. Jakšić 22/05/1985, 5, n.spec. = 1); Obilić: Donja Slatina (v.) (leg. G. Džukić 05/07/1969, 22, n.spec. =1); Metohia Dečani: Jasić (v.), Junička Reka (r.) (leg. G. Džukić, 04/06/1977, 23, n.spec. = 2); Istok: Istočka (r.) - spring (leg. anonim. 06/06/1977, 23, n.spec. = 3); Prizren: Vrbnica (v.) (leg. G. Džukić 07/07/1969, 23, n.spec. = 1); North-eastern Serbia Beljanica Mt.: Sokolica, near mountain house (leg. B. Žiljak, 12/10/1996, 8, n.spec. =1); Despotovac: Dvorište (v.) (leg. G. Džukić, M. Kalezić 12/05/2002, 8, n.spec. = 1); Đerdapska klisura gorge: Cuce (leg. BID "Josif Pančić" 23/04/1978, 26, n.spec. = 2; 23/07/1978, 26); (leg. BID “Josif Pančić" 23/04/1978, 26, n.spec. = 2); Kladovo: Korbovo (v.), Surduk (leg. G. Džukić, M. Kalezić 13/04/2008, 8, n.spec. = 1); Kučevo: Blagojev Kamen (v.) (leg. G. Džukić 07/06/1972, 26, n.spec. = 2); Malinik Mt.: Zlot (v.), Lazareva pećina cave - Vernjikica (leg. G. Džukić 08/07/1983, 26, n.spec. = 1); Negotin: Jabukovac (v.), Zamna (r.) (leg. G. Džukić, M. Kalezić 28/09/2005, 8, n.spec. = 1); Radujevac (v.) (leg. anonim. 13/05/1970, 8, n.spec. =1); (leg. G. Džukić 03/06/1972, 8, n.spec. =1); Zamna (r.) - canyon, Štubički Drenjar (leg. anonim. 21/04/1988, 8, n.spec. =2); North-western Serbia Ljubovija: Duvanište (v.) (leg. J. Crnobrnja Isailović, I. Aleksić, 22/03/1999, 4, n.spec. = 1); Gornja Trešnjica (v.), Osječenica (leg. S. Marinković 06/06/1991, 4, n.spec. = 1); Uzovnica (v.), Veleš (v.) (leg. BID "Josif Pančić" 02/07/1978, 4, n.spec. = 1; 03/08/1978, 4); Mionica: Paštrić (v.) (leg. G. Džukić 28/05/1980, 4, n.spec. =2); Povlen Mt.: Debelo Brdo hill (leg. anonim. s. d., 4, n.spec. = 3); Valjevo: Mravinjci (v.) (leg. Đ. Đoković 01/04/2004, 4, n.spec. = 1); Pomoravlje Golubac: fortress (leg. V. Vasić, 29/04/1972, 6, n.spec. = 1); Juhor Mt.: Kolare (v.) (leg. BID "Josif Pančić" 06/07/1984, 6, n.spec. = 2); Veliko Gradište: Srebrno jezero Lake (leg. G. Džukić, M. Kalezić 14.04. \& 15.04.1994, 6, n.spec. = 11); (leg. M. Kalezić 23/04/1995, 6, n.spec. = 2); South-eastern Serbia Besna Kobila Mt.: Musulj (v.), Crna Reka (r.) (leg. G. Džukić, M. Kalezić, M. Marković, 25/09/2005, 102, n.spec. = 1); Starac Mt.: Monastery Prohor Pčinjski, Gornji Starac (v.) (leg. G. Džukić, M. Kalezić, J. Crnobrnja Isailović 20.06. \& 21.06.1996, 7, n.spec. =4); (leg. G. Džukić, M. Kalezić 21/08/1994, 7, n.spec. = 7); (leg. Lj. Tomović, $R$. Ajtić 27/04/2000, 7, n.spec. =1; 28/04/2000, 7; Easter 2004, 7); (leg. M. Kalezić 17/06/2009, 7, n.spec. = 1); Starac (v.), Čivčije (h.), granica (leg. Lj. Tomović, R. Ajtić 06/05/2002, 7, n.spec. = 1); Surdulica: Klisura (v.), Groznatovci (v.), Jerma (r.) - gorge (leg. G. Džukić 19/05/1978, 102, n.spec. = 1); Trgovište: Crnovska Reka (r.) (leg. Lj. Tomović, R. Ajtić 29/04/2000, 102, n.spec. = 1; 03/05/2002, 102); Šaince (v.) (leg. G. Džukić 18/06/2009, 102, n.spec. = 1); Vražji Kamen (leg. J. Crnobrnja Isailović, I. Aleksić 04/06/1998, 102, n.spec. =1); Southern Serbia Preševo: Orahovica hill (leg. G. Džukić, 13/05/1977, 25, n.spec. =1); Starac Mt.: Biljača (v.) (leg. G. Džukić 17/07/1978, 25, n.spec. = 1); Vranje: surrounding (leg. D. Nedeljković 07/08/1958, 25, n.spec. = 2); Srem Fruška Gora Mt.: Andrevlje (leg. G. Džukić, 12/05/1975, 150, n.spec. =1; 16/05/1975, 150; May 1975, 150); Čortanovci (v.) (leg. G. Džukić 05/06/1978, 150, n.spec. = 3); Koševac (leg. G. Džukić 21/05/1974, 150, n.spec. = 1); Ležimir (leg. anonim. 20.05.-25.05.1982, 150, n.spec. = 1); Inđija: Slankamen (v.) (leg. G. Džukić 10/07/1974, 150, n.spec. = 2); Pećinci: Obrež (v.), Obedska bara pond (leg. anonim. 30/10/2004, 150, n.spec. = 1); Obedska bara pond, Matijevica (leg. anonim. 17/04/1970, 150, n.spec. = 1); Šumadija Avala Mt. (leg. A. Ćetković, 17/05/1987, 15, n.spec. =1); (leg. D. Pavićević 16/09/1984, 15, n.spec. =1); Beograd: Palilula, Višnjica (leg. D. Bejaković 24/05/1994, 15, n.spec. = 5); (leg. T. Vukov, A. Urošević, K. Ljubisavljević 16/04/2010, 15, n.spec. =1); Višnjica, Milićevo brdo hill (leg. G. Džukić 23/04/1970, 15, n.spec. = 1); Umka, Duboko (leg. M. Milenković 28/10/2001, 15, n.spec. = 1); Gornji Milanovac: Beršići (v.) (leg. anonim. 01/06/1997, 15, n.spec. = 1); Western Serbia Požega: Skrapež (r.) (leg. BID "Josif Pančić”, 06/07/1983, 781, n.spec. =1); Zlatibor Mt.: Gaj (v.), mouth of 
Dobroselička reka (r.) to Uvac (r.) (leg. M. Gvozdić 31/07/1992, 781, n.spec. =1); surrounding (leg. $M$. Gvozdić 02/05/1992, 781, n.spec. = 3); Slovenia Kranj Sveti Jošt (leg. B. Kryštufek, 25/04/1978, 780, n.spec. $=3$ ).

\section{Ophisops elegans}

Libya Cyrenaica Gegap (leg. A. Ćetković, 07/06/1988, 185, n.spec. =1).

\section{Podarcis erhardii}

Macedonia Pelagonia Bitolj: Lera (v.) (leg. G. Džukić, 24/07/1979, 121, n.spec. =10); Suvodol (v.) (leg. G. Džukić 25/07/1979, 121, n.spec. =2); Southern Mountains Mariovo: Dunje (v.) (leg. G. Džukić, 28/05/2004, 163, n.spec. = 12); Vitolište (v.) (leg. G. Džukić 28/05/2004, 163, n.spec. = 2); South-western Region Ohridsko jezero Lake: Ohrid (leg. anonim., 24/07/1951, 191, n.spec. $=24$ ); Prespansko jezero Lake: Pretor, Monastery Sveta Bogorodica (leg. A. Ćetković 11/08/1990, 121, n.spec. = 3); Serbia Kosovo Novo Brdo hill: Jasenovik (v.) (leg. P. Jakšić, 10/04/1986, 99, n.spec. =7); South-eastern Serbia Kozjak Mt.: Monastery Prohor Pčinjski (leg. G. Džukić, M. Kalezić, K. Ljubisavljević, 17/06/2009, 162, n.spec. = 20); (leg. G. Džukić, M. Kalezić, T. Vukov, K. Ljubisavljević 17.06. \& 18.06.2009, 146, n.spec. = 2); (leg. J. Crnobrnja Isailović, I. Aleksić 04/06/1998, 146, n.spec. =1); Starac Mt.: Monastery Prohor Pčinjski (leg. G. Džukić, M. Kalezić 21/08/1994, 146, n.spec. = 2); Starac (v.), Čivčije (h.), granica (leg. Lj. Tomović, R. Ajtić 06/05/2002, 146, n.spec. = 4); Trgovište: Crnovska Reka (r.) (leg. I. Medenica, M. Plećaš 20/04/2007, 138, n.spec. = 29); (leg. Lj. Tomović, R. Ajtić 18.06. \& 19.06.2006; 27.04.-29.04.2000, 137, n.spec. $=60$ ); Šaince (v.), above (leg. P. Jakšić 18/05/1985, 146, n.spec. = 3).

\section{Podarcis melisellensis}

S. I. (leg. anonim. s. d., 1600, n.spec. $=34$ ); Bosnia \& Herzegovina Herzegovina Mostar: Mostarsko Blato (leg. Denis Bem, 23/04/1988, 97, n.spec. = 3); Posušje: Poklečani (v.) (leg. G. Džukić 22/06/1971, 97, n.spec. = 1;864); Croatia Central Dalmatia Biševo (i.) (leg. anonim., 07/05/1950, 832, n.spec. $=15$ ); Brusnik (i.) (leg. anonim. 17/08/1947, 792, n.spec. $=16$; 09/05/1950, 824; 1953, 792); (leg. D. Novak 10/07/1954, 792, n.spec. = 1); Drvenik Veli (i.) (leg. anonim. 28/08/1951, 819, n.spec. $=5$ ); Jabuka (i.) (leg. anonim. 22/05/1950, 853, n.spec. $=21$ ); Korčula (i.): Lumbarda (leg. anonim. 18/07/1969, 761, n.spec. =1); Lastovo (i.) (leg. V. Vasić July 1966, 761, n.spec. = 1); Mali Rutenjak (i.) (leg. M. Radovanović 12/05/1950, 761, n.spec. = 2); Pakleni Otoci: Sveti Jerolim (i.) (leg. M. Radovanović 09/08/1951, 772, n.spec. =4); Sveti Klement (i.), Palmižana (leg. anonim. 07/08/1951, 772, n.spec. =1); Split - Vrgorac: Kozica (v.), Šibenik hill (leg. N. Tvrtković 29/08/1976, 782, n.spec. = 3); Vis (i.): Komiža (leg. M. Milenković 07/09/1986, 858, n.spec. = 2); Mali Paržanj (i.) (leg. anonim. 10/05/1950, 798, n.spec. = 14); (leg. M. Radovanović s. d., 874, n.spec. = 15); Vis (leg. M. Milenković 11/09/1986, 858, n.spec. =2); (leg. anonim. 06/05/1950, 858, n.spec. $=15$ ); Istria Grebeni (leg. anonim., 14/08/1953, 854, n.spec. =1); Pula (leg. anonim. 01/04/1991, 854, n.spec. =1); Rovinj: Šorići (v.) (leg. G. Džukić 11/04/1969, 854, n.spec. = 14); Tramerka (leg. anonim. 15/08/1953, 854, n.spec. $=1$ ); Kvarner Cres (i.): Merag port (leg. anonim., 16/04/1964, 800, n.spec. = 2); (leg. M. Kalezić 03/04/1991, 800, n.spec. = 17); Krk (i.) (leg. D. Jelić, S. Marinković s. d., 868, n.spec. = 2); Lošinj (i.): Čunski (leg. anonim. 23/09/1964, 868, n.spec. $=3$ ); Northern Dalmatia Crikvenica: Selce (v.) (leg. anonim., 22/04/1964, 799, n.spec. = 4); Dugi Otok (i.): Mala Skala (leg. anonim. 31/08/1956, 812, n.spec. = 3); Ravna Luka (leg. anonim. 31/08/1956, 812, n.spec. = 13); Rončić (i.) (leg. anonim. 31/08/1956, 812, n.spec. =4); Golac (i.): Uljan (leg. anonim. 07/08/1952, 861, n.spec. =1); Kaprije (i.): Blitvenica (i.) (leg. anonim. 20/08/1965, 863, n.spec. = 2); Mali Kamenišnjak (i.) (leg. anonim. 16/05/1965, 863, n.spec. = 1); (leg. anonim. 30/08/1966, 863, n.spec. = 14); Karantun (i.): Uljan (leg. anonim. 07/08/1952, 861, n.spec. $=3$ ); Kornati: Babina Guzica (i.) (leg. anonim. 17/08/1957, 849, n.spec. =27); Božikovac (i.) (leg. M. Radovanović 10/08/1952, 763, n.spec. =2); Buha (i.) (leg. M. Radovanović 14/09/1961, 763, n.spec. = 5); Gominjak (i.) (leg. anonim. 30/08/1956, 763, n.spec. = 1); Jančar (i.) (leg. anonim. 30/08/1956, 
763, n.spec. =2); Mrtovnjak (i.) (leg. M. Radovanović s. d., 846, n.spec. = 3); Mrtvac (i.) (leg. anonim. 27/08/1956, 846, n.spec. = 10); Opuh Mali (i.) (leg. anonim. 24/10/1951, 785, n.spec. = 12; 14/09/1961, 785); Opuh Veliki (i.) (leg. M. Radovanović 14/09/1961, 757, n.spec. = 10); Skrižanj Mali (i.) (leg. anonim. 14/09/1961, 852, n.spec. = 15); Skrižanj Veli (i.) (leg. anonim. 14/09/1961, 763, n.spec. =4); Trimulić Veli (i.) (leg. anonim. 10/08/1952, 825, n.spec. = 1); Velika Sestrica (i.), Vodice (leg. anonim. 27/07/1967, 825, n.spec. =8); Zornik (i.) (leg. M. Radovanović 27/08/1956, 756, n.spec. = 9); Žut (i.), Brusna (leg. anonim. 28/05/1959, 790, n.spec. = 2); Dajna (i.) (leg. anonim. 28/05/1959, 860, n.spec. =1); Dajnica (i.) (leg. anonim. July 1964, 790, n.spec. =1); Donja Dajnica (i.) (leg. anonim. 28/05/1959, 850, n.spec. = 10); Gornja Dajnica (i.) (leg. M. Radovanović 28/05/1959, 755, n.spec. $=9$ ); Mali Obrvan (i.) (leg. anonim. 28/05/1959, 860, n.spec. = 5); Obrvan (i.) (leg. anonim. 28/05/1959, 860, n.spec. = 2); Svršata Vela (i.) (leg. anonim. 30/05/1959, 790, n.spec. =1); Trbuh (v.) (leg. M. Radovanović 27/08/1956, 763, n.spec. =1); (leg. anonim. 30/05/1959, 763, n.spec. =1); Molat (i.): Obljak (i.) (leg. anonim. 14/08/1953, 844, n.spec. = 3); Tovarnjak (i.), Tijat, Vodice (leg. $M$. Radovanović 24/07/1967, 844, n.spec. =4); Olib (i.): Kurjak (s.) (leg. anonim. 01/09/1967, 786, n.spec. = 22); Pašman (i.): Košarica (i.) (leg. anonim. 17/08/1956, 861, n.spec. =1); Žižanj (i.) (leg. anonim. 16/08/1958, 861, n.spec. = 1); Pirovac (leg. Lj. Livajić 19/08/1988, 782, n.spec. = 2); Rivanj (i.): Fidula (leg. M. Radovanović 24/08/1955, 828, n.spec. =4); Ugljan (i.): Ugljan (v.), Lukoran (leg. M. Radovanović June 1960, 851, n.spec. = 13); (leg. anonim. 07/08/1956, 851, n.spec. = 3); Vrtlić (i.) (leg. anonim. 15/08/1957, 862, n.spec. = 8); Zadar: Kono (leg. anonim. June 1960, 816, n.spec. = 12); Žirje (i.): Blitvenica (s.) (leg. anonim. 21/10/1951, 783, n.spec. = 4); Kosmerka (i.) (leg. anonim. 19/08/1965, 774, n.spec. = 1); Mažirina (i.) (leg. anonim. 20/08/1965, 774, n.spec. =1); Sedlo (i.) (leg. anonim. Juy 1964, 787, n.spec. = 5); Školj od Mikavice (i.) (leg. anonim. 19/08/1951, 787, n.spec. = 19); Vrtlac (i.) (leg. anonim. 19/08/1965, 769, n.spec. =2); (leg. M. Radovanović s. d., 769, n.spec. = 15); (leg. anonim. 17/08/1951, 774, n.spec. =9; 20/10/1951, 774); Southern Dalmatia Dubrovnik: Sveti Andrija (i.) (leg. anonim., 10/08/1953, 789, n.spec. =1); (leg. M. Radovanović s. d., 789, n.spec. = 8); Lopud (i.) (leg. M. Radovanović 14/09/1954, 797, n.spec. = 3); Mljet (i.): Korita (leg. anonim. 02/05/1990, 892, n.spec. =1); Malo jezero Lake (leg. anonim. 03/05/1990, 892, n.spec. =1); Polačino Polje (leg. anonim. 03/05/1990, 892, n.spec. = 1); Saplunara (leg. anonim. 02/05/1990, 892, n.spec. = 4); Soline (leg. anonim. 04/05/1990, 892, n.spec. = 1); Velika Poma (leg. anonim. 01/04/1990, 892, n.spec. $=2$; 01/05/1990, 892); Veliko jezero Lake (leg. anonim. 04/05/1990, 892, n.spec. = 1); Zagora Ervenik: Pajića lokva (leg. G. Džukić, 01/06/1985, 843, n.spec. =1); Montenegro Central Montenegro Prekornica Mt.: Acrdani, Dolovi (leg. G. Džukić, M. Kalezić, M. Marković, s. d., 125, n.spec. = 2); Mediterranean Montenegro Boka Kotorska: Donji Morinj (v.) (leg. G. Džukić, M. Kalezić, 13/10/1996, 815, n.spec. =6); Mamula (i.) (leg. anonim. August 1994, 814, n.spec. = 5); (leg. M. Kalezić 06/08/1994, 814, n.spec. =27); Budva: Jaz (r.), $1 \mathrm{~km}$ upstream from the mouth (leg. A. Hegediš 01/08/1990, 796, n.spec. = 1); beach (leg. A. Ćetković 22/05/1995, 796, n.spec. = 5); Herceg Novi: Lastavica (i.), Mamula fortress (leg. J. Crnobrnja 02/07/1987, 807, n.spec. = 3); Savina, Monastery Savina (leg. G. Džukić 16/07/1969, 807, n.spec. = 3); Topla (leg. G. Džukić 18/07/1969, 807, n.spec. =1); Rumija Mt.: Sutorman, before the bend toward Bar (leg. A. Ivanović, M. Kalezić, I. Aleksić 29/05/1995, 796, n.spec. = 7); Tivat: Grbaljsko Polje, Tivatske solane (leg. anonim. 04/07/1992, 118, n.spec. = 1); Lastva Grbaljska, Donja Lastva (leg. anonim. 29/05/1996, 118, n.spec. =1); Mrčevsko Polje (leg. anonim. 27/05/1996, 118, n.spec. =2); Tivatske solane (leg. anonim. 13/07/1969, 118, n.spec. =2); Kulina (leg. anonim. 29/05/1996, 118, n.spec. =1); military hotel (leg. anonim. 29/05/1996, 118, n.spec. =2); (leg. anonim. 29/05/1996, 118, n.spec. = 2); Ulcinj: Ada Bojana (leg. I. Aleksić 17/09/1994, 817, n.spec. = 5); Bojana (r.) - mouth, Vada (i.) (leg. anonim. 02/04/1989, 818, n.spec. = 22); (leg. V. Vasić 09/07/1974, 818, n.spec. = 3); Velika Plaža (leg. A. Ćetković 06/09/1991, 817, n.spec. = 1); Submediterranean Montenegro Cetinje (leg. anonim., 15/07/1947, 116, n.spec. = 1); Cetinje (Ćeklići): Kućišta (v.), Crno Korito (leg. G. Džukić, M. Kalezić, M. Marković 20/06/2004, 116, n.spec. = 1); Ublica (leg. G. Džukić, M. Kalezić, M. Marković 20/06/2004, 116, n.spec. =2); Danilovgrad: Garač Mt. (leg. G. Džukić, M. Kalezić, M. Marković 29/05/2003, 845, n.spec. = 2); (leg. anonim. November 1951, 845, n.spec. = 8); Lovćen Mt.: Bjeloši (v.), Lašor (leg. G. Džukić, M. Kalezić, J. Crnobrnja, I. Aleksić 27/05/1996, 116, n.spec. =1); Orjen Mt.: Kamenica (v.), Borići (leg. G. Džukić, M. Kalezić 25/08/2001, 116, n.spec. =2); Podgorica: Ćemovsko Polje (leg. G. Džukić 10/03/1974, 842, n.spec. = 7); Releza (v.), Razvađa (leg. G. Džukić, M. Kalezić 22/08/2001, 842, n.spec. =1); Tuzi (v.) (leg. G. Džukić, M. Kalezić 11/10/1996, 842, n.spec. =1); Skadarsko jezero 
Lake: Bisage (i.) (leg. G. Džukić, M. Kalezić, D. Bejaković, I. Aleksić, J. Crnobrnja Isailović 16/05/1994, 836, n.spec. =7); Gorica III (i.) (leg. anonim. 08/05/1992, 911, n.spec. $=5$; 09/05/1992, 911); Gornji Murići (v.), above (leg. M. Kalezić, A. Ivanović, I. Aleksić 30/05/1995, 911, n.spec. =2); Lesendro (i.) (leg. G. Džukić 11/03/1974, 820, n.spec. = 8); Mala Beška (i.) (leg. anonim. s. d., 830, n.spec. = 37); Mala Čakovica (i.) (leg. G. Džukić 08/06/1980, 839, n.spec. =1); (leg. G. Džukić, M. Kalezić, D. Bejaković, I. Aleksić, J. Crnobrnja Isailović 17/05/1994, 839, n.spec. = 16); Moračnik Mali (i.) (leg. anonim. s. d., 826, n.spec. = 27); Starčevo (i.) (leg. anonim. June 1990, 822, n.spec. =6); (leg. G. Džukić 27/07/1975, 822, n.spec. = 3); Vranjina Peninsula (leg. G. Džukić, M. Kalezić, D. Bejaković, I. Aleksić, J. Crnobrnja Isailović 17/05/1994, 841, n.spec. = 8); Zeta: Bistrica (leg. anonim. 17/07/1992, 823, n.spec. = 47); Western Montenegro Nikšić: Koprivice (v.), Čista Vlaka (leg. G. Džukić, M. Kalezić, M. Marković, 11/06/2005, 125, n.spec. = 1); Vilusi (v.) (leg. G. Džukić 08/08/1970, 125, n.spec. $=4 ; 10 / 08 / 1974,125)$.

\section{Podarcis muralis}

S. I. (leg. anonim. s. d., 1596 , n.spec. $=43 ; 1597 ; 1598$ ); Albania Ohridsko jezero Lake Podgradec (leg. V. Pešić, 27/08/2002, 144, n.spec. =6); Bosnia \& Herzegovina Bosnia Drvar: Titova pećina cave (leg. V. Stevanović, 24/08/1983, 1583, n.spec. =1); Jajce (leg. B. Kryštufek 22/08/1972, 1583, n.spec. = 3); Šipovo: Janja (r.) - canyon (leg. Lj. Tomović, R. Ajtić 03.08.$-15.08 .2001,1583$, n.spec. = 4); Herzegovina Gacko: Bjelašnica Mt., Mangrop (leg. G. Džukić, 05/08/1970, 1583, n.spec. = 2); Martin Brod: Unac (r.) (leg. N. Tvrtković 26/05/1977, 1583, n.spec. = 1); Croatia Baranja Draž: Batina (v.), near the monument (leg. G. Džukić, 09/04/1972, 1533, n.spec. = 1); Central Dalmatia Biokovo Mt.: Sveti Jure (leg. anonim., 23/07/1969, 1534, n.spec. = 3); Istria Pula: city (leg. BID “Josif Pančić", 25/08/1976, 96, n.spec. = 1); (leg. anonim. 01/04/1991, 96, n.spec. = 17); Rovinj (leg. anonim. 12/04/1969, 96, n.spec. =2); Kvarner Cres (i.) (leg. anonim., August 1990, 1532, n.spec. = 14); Lika Gračac (leg. G. Džukić, 01/06/1985, 1531, n.spec. =1); Velebit Mt.: Kaplja, Visibaba (leg. N. Tvrtković 05/08/1977, 1534, n.spec. =1); Predzid (leg. N. Tvrtković 04/07/1975, 1534, n.spec. = 1); Vrhovine: Dugi Do, Dugi Do, above (leg. anonim. 10/10/1961, 1531, n.spec. = 3); (leg. anonim. 10/10/1961, 1531, n.spec. =2); Slavonia Papuk Mt.: Jankovac (leg. G. Džukić, 06.05.-08.05.1981, 1533, n.spec. =11); Macedonia Central Mountains Babuna Mt.: Derven (leg. G. Džukić, 10/05/1972, 1535, n.spec. = 3); Eastern Mountains Berovo: Ratevska Reka (r.) (leg. anonim., 26/05/1970, 1538, n.spec. = 8); Kratovo: Kratovska Reka (r.) (leg. anonim. 12/08/1982, 1538, n.spec. =1); Stari Grad (leg. anonim. 12/08/1982, 1538, n.spec. =1); Southern Mountains Kožuf Mt.: Konsko (v.) (leg. anonim., 18/07/1990, 1536, n.spec. = 2); Nidže Mt.: Kajmakčalan (leg. M. Belanović 26/09/1972, 1536, n.spec. = 11); South-western Region Galičica Mt.: bend (leg. G. Džukić, 20/09/1978, 1539, n.spec. =6); Ohridsko jezero Lake: Ohrid (leg. anonim. 24/07/1951, 1539, n.spec. = 4); (leg. S. Brelih, B. Kryštufek 04/11/1972, 1539, n.spec. =2); Peštani (v.) - Trpejca (v.) (leg. G. Džukić 18/09/1978, 1539, n.spec. = 12); Sveti Naum, Biljanini izvori (leg. G. Džukić 17/05/1980, 1539, n.spec. =1); Pelister Mt.: Nižepole (leg. A. Ćetković 08/08/1990, 1539, n.spec. = 2); Prespansko jezero Lake: Oteševo (v.) (leg. G. Džukić 19/09/1978, 1539, n.spec. = 7; 18/05/1980, 1539); Pretor, Monastery Sveta Bogorodica (leg. anonim. 11/08/1990, 1539, n.spec. =1); Western Mountains Radika (r.) - canyon (leg. G. Džukić, 21/09/1978, 1535, n.spec. = 2); Montenegro Central Montenegro Donja Morača (leg. G. Džukić, 10/07/1969, 1559, n.spec. = 1); Mrtvica (r.) - canyon (leg. R. Ajtić 27/07/2001, 1559, n.spec. = 1); Eastern Montenegro Bjelasica Mt.: Biogradska Gora, Ostrvica (leg. G. Džukić, M. Kalezić, 24/08/1994, 148, n.spec. =1); Đebeza Mt.: bend (leg. G. Džukić, M. Kalezić, M. Marković 28/05/2003, 148, n.spec. = 1); southern slopes (leg. G. Džukić, M. Kalezić, M. Marković 28/05/2003, 148, n.spec. =1); Kolašin: road toward Andrijevica (leg. V. Vasić 12/07/1974, 148, n.spec. =1); Lim (r.) - gorge: Murino - Andrijevica (leg. G. Džukić 08/07/1969, 148, n.spec. = 1); Prokletije Mts.: Gusinje (leg. G. Džukić 22/09/1969, 148, n.spec. = 3); Jezerce, 3 km toward Jezerce (leg. G. Džukić 02/07/1984, 148, n.spec. =1); Rikavačko jezero Lake (leg. G. Džukić 20/09/1991, 148, n.spec. =1); Mediterranean Montenegro Ulcinj: Ada Bojana, bridge (leg. G. Džukić, M. Kalezić, M. Marković, 08/06/2005, 776, n.spec. =10); (leg. A. Ćetković 07/09/1991, 776, n.spec. = 1); (leg. anonim. 06/08/2005, 776, n.spec. = 1); Northern Montenegro Durmitor Mt.: Crno jezero Lake, Čeline (leg. P. Jakšić, 02/07/1987, 885, n.spec. =1); 
Kutnja Njiva (leg. anonim. 08/01/1984, 885, n.spec. = 1); Zminje jezero Lake (leg. BID "Josif Pančić" 10/07/1979, 885, n.spec. =1); Tara (r.) - canyon: Budečevica, Splavište (leg. G. Džukić 26/06/1985, 885, n.spec. = 3); (leg. P. Jakšić 24/06/1985, 885, n.spec. =1); Dobrilovina (leg. anonim. 28/07/1990, 885, n.spec. = 1); Tepca (v.), Devojački most (leg. G. Džukić 04/07/1986, 885, n.spec. = 2); (leg. anonim. 14/09/1990, 885, n.spec. = 2); Submediterranean Montenegro Cetinje: Trnjine (v.), Bijele Poljane (v.) (leg. G. Džukić, M. Kalezić, M. Marković, 29/05/2003, 113, n.spec. = 1); Male Cuce (leg. G. Džukić, M. Kalezić, M. Marković 29/05/2003, 113, n.spec. = 1); Cetinje (Ćeklići): Kućišta (v.), Crno Korito (leg. G. Džukić, M. Kalezić, M. Marković 20/06/2004, 113, n.spec. = 3); Vučji Do (v.) (leg. G. Džukić, M. Kalezić, M. Marković 26/06/2004, 113, n.spec. =1); Lovćen Mt.: Jezero (leg. M. Kalezić 17/08/1991, 113, n.spec. = 3); Orjen Mt.: Kamenica (v.), Borići (leg. anonim. 25/08/2001, 117, n.spec. =1); Mala Siljevica (leg. M. Milenković 02/06/1979, 117, n.spec. = 2); Risan - v. Donje Crkvice (leg. V. Vasić 07/07/1974, 117, n.spec. =4); Vrbanje (v.) (leg. G. Džukić 15/06/1972, 117, n.spec. = 1); Skadarsko jezero Lake: Gorica Gjat (i.) (leg. anonim. s. d., 83, n.spec. $=31$; 1580); Gorica I (i.) (leg. anonim. 10/10/1992, 1586, n.spec. =24; 11/10/1992, 809); Gorica Skurt (i.) (leg. anonim. s. d., 1548 , n.spec. = 73); Gradac (i.) (leg. anonim. s. d., 1549, n.spec. =61); Grmožur (i.) (leg. anonim. 12/09/1991, 898, n.spec. = 2); Kalundrica (i.) (leg. anonim. s. d., 1550, n.spec. =97; 1551); (leg. G. Džukić, M. Kalezić, D. Bejaković, I. Aleksić, J. Crnobrnja, E. Kletečki, N. De Luca 06/06/1990, 1551, n.spec. $=1$; 05.06. \& 06.06.1990, 1551); Krš od Starčeva (i.) (leg. anonim. June 1990, 1545, n.spec. = 7; s. d., 1545); Lesendro (i.) (leg. anonim. s. d., 1552, n.spec. =36); Livari (v.), Briska, Maskodra (leg. G. Džukić, M. Kalezić 12/10/1996, 898, n.spec. =1); Malo Starčevo (i.) (leg. anonim. 1990, 1553, n.spec. =63); Moračnik (i.) (leg. anonim. s. d., 1547, n.spec. = 36); Murići (v.), toward Virpazar (leg. anonim. 07/06/1991, 898, n.spec. = 2); Omerova Gorica (i.) (leg. anonim. 09/05/1992, 811, n.spec. =10; 1585; 15/07/1992, 1588; 1990, 1554; May 1992, 1587); Plavnica (leg. anonim. 17/07/1992, 1556, n.spec. = 38; s. d., 1557); Zeta: Bistrica (leg. anonim. 11/05/1992, 1540, n.spec. $=12 ; 11.05$. \& 17.07.1992, 1540); Western Montenegro Nikšić: Koprivice (v.), Čista Vlaka (leg. anonim., 11/06/2005, 120, n.spec. = 1); Velika Osječenica (v.) (leg. anonim. 23/08/1982, 120, n.spec. = 5); Vilusi (v.), Ćetkova Kamenica (leg. G. Džukić, M. Kalezić, M. Marković 25/06/2004, 120, n.spec. = 2); Donja Spila, Voluje oko (leg. G. Džukić, M. Kalezić, M. Marković 25/06/2004, 120, n.spec. = 1); (leg. G. Džukić 08/08/1970, 120, n.spec. = 2); Njegoš Mt.: Gornja Trepča (v.), Roganovića Voda (leg. G. Džukić, M. Kalezić 08/06/2008, 120, n.spec. = 1); Orjen Mt.: Grahovo, dam (leg. anonim. 25/05/1996, 120, n.spec. = 1); Serbia Banat Beograd: Palilula, Borča, Mali Zbeg (leg. L. Đurđević, 05/05/1996, 1541, n.spec. = 1); Deliblatska peščara: Deliblato (v.) (leg. anonim. 10/06/1948, 1541, n.spec. = 1); Kovin: city (leg. anonim. 26/04/1969, 1541, n.spec. = 10); (leg. G. Džukić 29/04/1969, 1541, n.spec. = 3); Vršački breg Mt.: Lisičja Glava (leg. G. Džukić 09/06/1970, 1541, n.spec. =1); Malo Središte (v.), toward Gudurički vrh (leg. G. Džukić, M. Kalezić 19/05/2003, 1541, n.spec. = 7); (leg. K. Ljubisavljević 27/06/2003, 1541, n.spec. = 1); Vršački Vrh (leg. G. Džukić 19/06/1969, 1541, n.spec. = 2); Central Serbia Jastrebac Mt.: Buci (v.) (leg. anonim., 17/10/1977, 1542, n.spec. =2); Loparda (leg. anonim. 26/06/1987, 1542, n.spec. =2); Kopaonik Mt.: Oštre Stene (leg. G. Džukić 12/05/1976, 1542, n.spec. =1); Samokovska Reka (r.) - gorge (leg. J. Crnobrnja 01/06/1986, 1542, n.spec. = 5); Savinac (leg. anonim. 24/07/1987, 1542, n.spec. = 1); Šanac (leg. G. Džukić 11/05/1976, 1542, n.spec. = 1); Prokuplje: Toplica (r.) (leg. M. Radovanović June 1961, 1542, n.spec. = 3); Eastern Serbia Pirot: Krupac (v.), Krupačko jezero Lake, Brkina Vrela (leg. G. Džukić, 23/05/1977, 1578, n.spec. = 11); Stara planina Mt.: Crni Vrh (leg. BID "Josif Pančić" 16/07/1983, 1578, n.spec. = 1); Crni Vrh (v.), toward v. Balta Berilovac (leg. J. Crnobrnja Isailović 15/07/1994, 1578, n.spec. =1); Crnovrška Reka (r.) (leg. A. Ćetković 14/07/1994, 1578, n.spec. =1); Rakitska Reka (r.) - valley, toward v. Topli Do (leg. J. Crnobrnja Isailović 17/07/1994, 1578, n.spec. =1); Vrelo (v.), Visok (leg. G. Džukić, M. Kalezić, D. Jović 04/07/2008, 1578, n.spec. = 1); Svrljig: Prekonoge (v.), Prekonoška pećina cave (leg. anonim. 13/05/1970, 1578, n.spec. = 1); Kosovo Gnjilane: Buzalak (v.), Dobri Čiča hill (leg. G. Džukić, 17/05/1977, 848, n.spec. = 4); Ibar (r.) - valley: Lozište (v.), Košutovački potok (leg. $P$. Jakšić 22/06/1986, 848, n.spec. = 5; 24/07/1987, 848); Novo Brdo hill: Bostane (v.), Bostanska (r.) (leg. P. Jakšić 15/04/1986, 1554, n.spec. = 11); (leg. P. Jakšić 08/10/1984, 1554, n.spec. = 2; 03/04/1986, 1554; 05.05. \& 14.05.1985, 1554); Čuljkovci (v.) (leg. P. Jakšić 15/05/1985, 1571, n.spec. =3); Duboki Potok (v.) (leg. P. Jakšić 12/04/1986, 1571, n.spec. = 8); Jasenovik (v.) (leg. P. Jakšić $15 / 05 / 1985$, 1564, n.spec. $=1 ; 10 / 04 / 1986,1564 ; 10.04 . \& 11.04 .1986,1564)$; Kaljaja (v.) (leg. $P$. Jakšić 15/05/1986, 1565, n.spec. = 29); Kladenac (v.) (leg. P. Jakšić 15/05/1985, 1571, n.spec. = 7); Klobukar (v.), Trnićevska Reka (r.) (leg. P. Jakšić 15/05/1985, 1566, n.spec. = 8); (leg. P. Jakšić $22 / 05 / 1985$, 1566, n.spec. $=23 ; 10 / 04 / 1986,1566$ ); Kriva Reka (r.) - spring (leg. P. Jakšić 03/04/1986, 1571, n.spec. = 1); Manišince (v.) (leg. P. Jakšić 22/05/1985, 1571, n.spec. = 1); 
Prekovce (v.) (leg. P. Jakšić 22/05/1985, 1571, n.spec. = 3); Šumaci (v.) (leg. P. Jakšić 15/05/1985, 1567 , n.spec. $=15 ; 10 / 04 / 1986,1567)$; Trnićevce (v.) (leg. P. Jakšić 15/05/1985, 1568, n.spec. $=5$; 27/05/1986, 1568); Zebince (v.), Kriva Reka (r.) (leg. P. Jakšić 15/05/1985, 1569, n.spec. = 16); (leg. P. Jakšić 05/05/1985, 1570, n.spec. $=4 ; 15 / 05 / 1985,1570 ; 22 / 05 / 1985,1570 ; 04 / 04 / 1986,1570)$; Obilić: surrounding (leg. G. Pasuljević April 1967, 848, n.spec. =1); Vučitrn: Samodreža (v.) (leg. G. Džukić 04/07/1969, 848, n.spec. =2); Metohia Dečani: Dečanska Bistrica $(\mathrm{r}$.) - gorge, $7 \mathrm{~km}$ upstream from Monastery Dečani (leg. V. Stevanović, 10/07/1977, 1579, n.spec. = 1); Jasić (v.), Junička Reka (r.) (leg. G. Džukić 04/06/1977, 1579, n.spec. = 11); Monastery Visoki Dečani (leg. G. Džukić 05/06/1977, 1579, n.spec. = 2); Istok: Istočka (r.) - spring (leg. G. Džukić 06/06/1977, 1579, n.spec. = 1); Peć: Miliševac spring (leg. P. Jakšić 16/08/1985, 1579, n.spec. =1); Prizren: Dečanska Bistrica (r.) - gorge, Vrbički potok (leg. V. Stevanović 09/07/1977, 1579, n.spec. =1); Prizrenska Bistirca (r.) (leg. P. Jakšić 07/07/1987, 1579, n.spec. = 2); (leg. V. Vasić 07/07/1969, 1579, n.spec. = 1); Prokletije Mts.: Radavac (v.), Beli Drim (r.) - spring (leg. G. Džukić 08/06/1977, 1577, n.spec. = 10); Ivanov potok (leg. G. Džukić 08/06/1977, 1577, n.spec. = 3); North-eastern Serbia Beljanica Mt.: Busovata, bellow Busovata (leg. anonim., 03/06/1979, 160, n.spec. =2); (leg. R. Ajtić July 1998, 160, n.spec. =2); Đerdapska klisura gorge: Cuce (leg. BID "Josif Pančić" 23/04/1978, 160, n.spec. = 1; 23/07/1978, 160); Kučajske planine Mts.: Grza (r.) - gorge, mountain house (leg. M. Rajić 08/07/2009, 160, n.spec. = 1); Malinik Mt.: Zlot (v.), Lazareva pećina cave - Vernjikica (leg. G. Džukić 08/07/1983, 1584, n.spec. = 9); (leg. BID “Josif Pančić” 02/05/1987, 1584, n.spec. =2); Negotin: Zamna (r.) (leg. G. Džukić, M. Kalezić 22/06/2003, 160, n.spec. = 8); Zaječar: Gamzigrad (leg. V. Vasić 01/05/1972, 160, n.spec. =1); Kraljevica (leg. G. Džukić 17/08/1981, 160, n.spec. =1); North-western Serbia Loznica (leg. BID “Josif Pančić, 27/03/1979, 1582, n.spec. =1); Ljubovija: Gornja Trešnjica (v.) (leg. G. Džukić 27/05/1972, 1582, n.spec. = 18); Maljen Mt.: Divčibare (leg. M. Plećaš 06/08/2002, 1582, n.spec. =1); Povlen Mt.: Debelo Brdo hill (leg. anonim. s. d., 1582, n.spec. = 3); Valjevo: Gradac (r.) - canyon (leg. G. Džukić 26/06/1983, 1582, n.spec. =1); (leg. BID "Josif Pančić" 19/03/1976, 1582, n.spec. = 1); Pomoravlje Juhor Mt.: Kolare (v.) (leg. BID "Josif Pančić", 07/07/1984, 1546, n.spec. = 1); (leg. BID "Josif Pančić" 13/07/1984, 1546, n.spec. = 1); Smederevo: Vranovo (v.) (leg. M. Marković 24/11/2003, 1546, n.spec. =1); South-eastern Serbia Bosilegrad: Donja Ljubata (v.) (leg. anonim., 25/09/2005, 161, n.spec. =1); Surdulica: Jelašnica (r.) - gorge (leg. J. Šoti 12/07/1978, 161, n.spec. = 1); Trgovište: Crnovska Reka (r.) (leg. Lj. Tomović, R. Ajtić 29/04/2000, 161, n.spec. = 3); Đavolja Glava (leg. G. Džukić, M. Kalezić, T. Vukov, K. Ljubisavijević 18/06/2009, 161, n.spec. $=20$ ); Southern Serbia Medveđa: Tulare (v.), Mrkonj planina Mt., Veliki Vis (leg. anonim., 16/05/1977, 1544, n.spec. = 3); Pčinja (r.) - valley: Šaince (v.), Trnićevska Reka (r.) (leg. P. Jakšić 18/05/1985, 1544, n.spec. = 2); Vranje: Markovo Kale (leg. G. Džukić 15/05/1977, 1544 , n.spec. $=12$ ); South-western Serbia Golija Mt.: Crepuljnik, Košaninovo jezero Lake (leg. G. Džukić, M. Kalezić, 21/08/2001, 1543, n.spec. =1); Mokra Gora Mt.: Crna Reka (r.), Monastery Crna Reka (leg. G. Džukić 11/06/1977, 1543, n.spec. =1); Nova Varoš: Radoinja (v.), Sjenište (v.) (leg. G. Džukić 10/08/1982, 1543, n.spec. = 1); Šupljica (v.), Neškoviće (leg. G. Džukić 11/08/1982, 1543, n.spec. =11); Srem Fruška Gora Mt.: Andrevlje, quarry (leg. G. Džukić, 11/05/1975, 1581, n.spec. = 1); road toward Testera (leg. G. Džukić 16/05/1975, 1581, n.spec. =1); (leg. G. Džukić May 1975, 1581, n.spec. =1); Crveni Čot (leg. anonim. 06/05/1969, 1581, n.spec. =1); Goli Bikol (leg. anonim. 06/05/1969, 1581, n.spec. =1); Koševac, Podčenta (leg. G. Džukić 10/07/1974, 1581, n.spec. =1); Osovlje (leg. B. Božičić 19/04/1980, 1581, n.spec. = 1); Šarengrad (leg. G. Džukić 15/04/1981, 1581, n.spec. = 3); Vrdnik (v.), Vrdnička Kula (leg. G. Džukić 19/03/1975, 1581, n.spec. = 1); Zmajevac (leg. G. Džukić 17/03/1975, 1581, n.spec. = 4; 18/03/1975, 1581); Zemun (leg. M. Cvijanović 28/03/2012, 1581, n.spec. = 1); Šumadija Avala Mt.: Čarapićev Brest (leg. anonim., 22/05/1992, 810, n.spec. $=11$ ); (leg. anonim. 19/04/1970, 810, n.spec. $=1$; 02/05/1992, 810); Beograd: Ada Ciganlija (i.), Makiš (leg. M. Đorđević 20/05/1959, 94, n.spec. =1); (leg. A. Ivanović 29/06/2009, 94, n.spec. $=1$ ); Čukarica, Košutnjak (leg. anonim. 09/05/1969, 94, n.spec. $=4$ ); (leg. K. Ljubisavljević 07/04/1997, 94, n.spec. = 1); Sremčica, Lipovička šuma (leg. anonim. 19/08/1986, 94, n.spec. $=1$ ); Palilula, Ada Huja, Višnjica, Pionir (leg. K. Ljubisavljević, D. Bejaković April - October 1994, 95, n.spec. = 196); Institute for Biological Research (leg. A. Urošević 19/06/2009, 94, n.spec. = 2); (leg. anonim. 19/08/1986, 94, n.spec. $=2 ; 14 / 07 / 2003$, 94); (leg. V. Vasić 26/05/1969, 94, n.spec. $=1$ ); Kalemegdan, Pionir, Institute for Biological Research (leg. leg majki: K. Ljubisavljević, T. Vukov, A. Urošević, lab rad: K. Ljubisavljević born in July \& August 2009, 93, n.spec. = 20); Karaburma, Institute for Biological Research (leg. G. Džukić 06/06/1974, 94, n.spec. =2); Pionir (leg. K. Ljubisavljević, T. Vukov 10/06/2009, 94, n.spec. = 14); Višnjica, Milićevo brdo hill (leg. G. Džukić 23/04/1970, 94, n.spec. 
= 1); Rakovica, Monastery Rakovica (leg. anonim. 09/05/1969, 94, n.spec. = 1); Stari Grad, Kalemegdan (leg. A. Urošević 11.06. \& 29.06.2009, 94, n.spec. = 3); Voždovac, Braće Jerković (leg. K. Ljubisavljević 19/06/2009, 94, n.spec. = 1); Zvezdara (leg. G. Džukić 24/03/1970, 94, n.spec. = 1); Western Serbia Kosjerić: Tubići (v.), Skrapež (r.) (leg. BID “Josif Pančić”, 03/07/1983, 1558, n.spec. = 1); Kraljevo: Savovo (v.), Studenica (r.), mouth of Savošnica (r.) (leg. V. Stevanović 13/07/1977, 1558, n.spec. = 5); Tara planina Mt.: Brusnica (leg. G. Džukić 12/06/1969, 1560, n.spec. =1); Derventa (r.) - canyon, Perućac Lake (leg. V. Vasić 21/04/1969, 1560, n.spec. = 1); Drina (r.) - valley, Perućac Lake (leg. G. Džukić 21/05/1972, 1560, n.spec. = 2); Jagoštica (v.), Galine (leg. anonim. 21/05/1972, 1560, n.spec. = 1); Vidača (leg. G. Džukić 23/05/1972, 1560, n.spec. = 1); Kaluđerske Bare (leg. G. Džukić 09/06/1969, 1560, n.spec. = 1); Mitrovac, Sumbilić hill (leg. G. Džukić 10/06/1969, 1560, n.spec. = 1); Rastište (v.), Požarh. (leg. G. Džukić 21/05/1972, 1560, n.spec. =4); Šljivovica (v.), Kaluđerske bare (leg. G. Džukić 21/06/1973, 1560, n.spec. = 1); Zaovine (v.), Beli Rzav, lake (leg. G. Džukić 07/05/1974, 1560, n.spec. = 1); Crni vrh, Čemerište (leg. G. Džukić 20/05/1972, 1560, n.spec. = 4); Užice: Stari Grad (leg. M. Gvozdić 15/03/1992, 1563, n.spec. = 1); Zlatibor Mt.: Gaj (v.), surrounding (leg. M. Gvozdić 23/04/1992, 1563, n.spec. = 8); Partizanske Vode (leg. K. Ljubisavljević 25/08/2003, 1563, n.spec. =2); (leg. M. Gvozdić 23/04/1992, 1563, n.spec. = 1); Tetrebovac, Crni Omar (leg. M. Gvozdić 27/07/1992, 1561, n.spec. = 22); foothill (leg. M. Gvozdić 28/07/1992, 1561, n.spec. = 5); Uvac (r.) - canyon (leg. M. Gvozdić 05/05/1992, 1561, n.spec. = 35); (leg. M. Gvozdić 28/07/1992, 1561, n.spec. = 4); Veliki Rzav (r.), surrounding (leg. M. Gvozdić 25/07/1992, 1562, n.spec. = 3); (leg. M. Gvozdić 01/05/1992, 1562, n.spec. =42); Slovenia Koper Semedela (leg. BID “Josif Pančić, 24/08/1976, 895, n.spec. = 1); Piran(leg. anonim., 03/04/1991, 895, n.spec. =6); Primorsko Vipava (r.) (leg. B. Kryštufek, 09/01/1973, 895, n.spec. = 1); Snežnik Mt.: Štirne (leg. anonim. 06/05/2005, 895, n.spec. $=1$ ).

\section{Podarcis peloponnesiacus \\ Greece Peloponnese Mykenai (leg. G. Džukić, 08/11/1972, 158, n.spec. =1).}

\section{Podarcis siculus}

S. I. (leg. anonim. s. d., 1599, n.spec. = 30); Bosnia \& Herzegovina Herzegovina Neretva - mouth (r.), Galičak (i.) (leg. anonim., 07/04/1989, 775, n.spec. $=27$ ); Croatia Central Dalmatia Lastovo (i.): Bjelac (i.) (leg. anonim., s. d., 829, n.spec. = 14); Kopište (i.) (leg. M. Radovanović s. d., 771, n.spec. = 5); Podmrčaru (s.) (leg. M. Radovanović 12/05/1950, 754, n.spec. = 26); Potkopište (i.) (leg. anonim. s. d., 821, n.spec. = 17); Mala Palagruža (i.) (leg. anonim. s. d., 834, n.spec. = 30); Palagruža (i.) (leg. M. Radovanović s. d., 758, n.spec. =12); Split: Bačvice (leg. anonim. 14/08/1951, 857, n.spec. =1); Čiovo (leg. anonim. 26/05/1979, 857, n.spec. =13); Trogir (leg. anonim. 27/05/1960, 857, n.spec. =2); (leg. anonim. May 1950, 857, n.spec. = 3); Istria Rovinj: Asino (leg. anonim., 25/08/1953, 867, n.spec. $=3$ ); Crveni Otok (i.) (leg. anonim. 10/07/1950, 870, n.spec. = 16; 29/10/1958, 870); (leg. D. Pelić 20/05/1975, 870, n.spec. = 5); Figarola Grande (i.) (leg. anonim. 21/08/1953, 872, n.spec. $=8 ; 24 / 08 / 1953,872$ ); Katarina (i.) (leg. D. Pelić 19/05/1975, 760, n.spec. $=$ 2); Longa (leg. anonim. 25/08/1953, 855, n.spec. = 9); Punte Korente (leg. D. Pelić 22/05/1975, 867, n.spec. = 2); Sveta Katarina (i.) (leg. S. Leiner May 1977, 760, n.spec. =1); Sveti Ivan (i.) (leg. M. Radovanović 26/08/1953, 869, n.spec. = 9); Šorići (v.) (leg. G. Džukić 11/04/1969, 847, n.spec. = 9); Velika Sestrica (i.) (leg. D. Pelić 21/05/1975, 760, n.spec. = 8); Kvarner Grujica (i.) (leg. anonim., 1953, 788 , n.spec. $=3$ ); Krk (i.): Klimno (leg. anonim. 19/04/1964, 788, n.spec. =2); Mali Laganj (i.) (leg. anonim. 15/08/1953, 831, n.spec. = 13); Mišnjak (i.): Uljan (leg. M. Radovanović 07/08/1952, 762, n.spec. = 5); Rab (i.): Palit (leg. D. Pelić 01/08/1975, 871, n.spec. =9); Sveti Đurađ (leg. anonim. 25/08/1951, 865, n.spec. = 10); Susak (i.) (leg. anonim. 21/08/1952, 899, n.spec. = 2); (leg. M. Pljakić 16/08/1960, 899, n.spec. = 7); Northern Dalmatia Biograd: Cavata (i.) (leg. M. Radovanović, 22/08/1955, 765, n.spec. = 2); Frmić (i.) (leg. M. Radovanović 22/08/1955, 765, n.spec. = 8); Gnalić (i.) (leg. anonim. 16/08/1958, 873, n.spec. =1); Kaprije (i.): Oštrica (i.) (leg. anonim. 28/05/1951, 835, n.spec. =3); Veliki Dupinić (i.) (leg. anonim. 28/10/1951, 835, n.spec. =14); Kornati: Bisage (i.), Uljan (leg. anonim. 07/08/1952, 767, n.spec. $=4$ ); Mala Sestrica (i.), near i. Ist (leg. M. Radovanović 17/08/1952, 767, n.spec. =1); (leg. anonim. 10/08/1952, 767, n.spec. $=1$ ); Murvenjak (i.) 
(leg. anonim. 15/08/1957, 840, n.spec. = 4); Prišnjak (i.) (leg. anonim. 15/08/1957, 767, n.spec. = 2); Purara (i.) (leg. anonim. 16/08/1957, 878, n.spec. $=28$ ); Veseljuh (i.) (leg. anonim. 28/08/1956, 767, n.spec. = 3); (leg. M. Radovanović 16/08/1957, 767, n.spec. = 3); Vrgada (i.) (leg. anonim. 15/08/1957, 840, n.spec. = 1); Mali Dupinić (i.) (leg. anonim. 28/10/1951, 859, n.spec. $=11$ ); Murter (i.): Čavlin (i.) (leg. M. Radovanović 17/08/1957, 770, n.spec. = 8); Visovac (i.) (leg. anonim. 15/08/1957, 770, n.spec. = 1); Olib (i.): Pohlib (i.) (leg. anonim. 31/08/1967, 837, n.spec. = 8); Šip - Prekošip (leg. anonim. 01/09/1967, 837, n.spec. = 2); Pakoštane: Sveta Juština (i.) (leg. anonim. 26/05/1959, 838, n.spec. $=3$ ); Školjić (i.) (leg. anonim. 26/05/1959, 838, n.spec. $=13$ ); Vransko jezero Lake (leg. anonim. 25/05/1959, 877, n.spec. =17); Pašman (i.): Bisaga Vela (i.) (leg. anonim. 14/08/1956, 813, n.spec. = 2); Galešnjak (i.) (leg. anonim. 14/08/1956, 813, n.spec. =3); Garmenjak (i.) (leg. anonim. 14/08/1956, 813, n.spec. =1); Mali Dušac (i.) (leg. anonim. 14/08/1956, 813, n.spec. = 4); Ričul (i.) (leg. anonim. 14/08/1956, 813, n.spec. = 7); Premuda (i.): Lutrošnjak (i.) (leg. anonim. 27/08/1955, 875, n.spec. =17); (leg. anonim. 26/08/1955, 856, n.spec. = 8); Rab (i.): Veliki Laganj (i.) (leg. $M$. Radovanović 15/08/1953, 764, n.spec. = 6); Rijeka: Kamenjak (leg. anonim. 17/08/1964, 873, n.spec. $=$ 2); Rivanj (i.): Tri Sestrice (i.), Srednji Školj (leg. anonim. 24/08/1955, 833, n.spec. =10); (leg. $M$. Radovanović 14/09/1953, 801, n.spec. $=12$ ); Šibenik (leg. M. Radovanović s. d., 768, n.spec. $=16$ ); Zadar: Ljubač (v.), Ljubač bay, from Zadar toward Pag (i.) (leg. V. Stevanović 01/08/1981, 920, n.spec. = 2); (leg. V. Stevanović 15/08/1984, 920, n.spec. = 1); Punta Radman, Peprčane (v.) (leg. V. Stevanović 13.08.-23.08.1983, 920, n.spec. = 6); Zemunik (v.) (leg. anonim. s. d., 879, n.spec. $=28$ ); Zadar - Biograd: Školj Ričul, Krnčina (v.) (leg. V. Stevanović 18/08/1984, 920, n.spec. =1); Žirje (i.): Gušteranski Školj (leg. anonim. 18/08/1951, 793, n.spec. $=2$ ); Koromašna Školj (leg. anonim. 18/08/1951, 793, n.spec. = 3; 21/08/1958, 793); Ravan (i.) (leg. M. Radovanović 20/08/1965, 793, n.spec. = 3); Zagora Ervenik: Pajića lokva (leg. G. Džukić, 01/06/1985, 888, n.spec. = 3); Obrovac: Biovičino Selo (v.) (leg. G. Džukić 31/05/1985, 888, n.spec. =1); Kolašac (v.), Matijevići (leg. G. Džukić 31/05/1985, 888, n.spec. =2); Italy Koper Salerno: Capaccio Scalo, Pestum (leg. G. Džukić, 07.06.-22.06.1978, 916, n.spec. = 7); Montenegro Mediterranean Montenegro Boka Kotorska: Mamula (i.) (leg. M. Kalezić, 30/07/1994, 153, n.spec. =6); Kotor: city (leg. anonim. 24/05/1996, 112, n.spec. =1); Dobrota (leg. G. Džukić, M. Kalezić, J. Crnobrnja Isailović, I. Aleksić 26.05. \& 27.05.1996, 103, n.spec. = 9); (leg. M. Kalezić, A. Ivanović, I. Aleksić 1995 \& 1996, 112, n.spec. = 2; 27.05. \& 28.05.1995, 112); (leg. anonim. 21/09/1954, 112, n.spec. =2); Slovenia Koper Gažon (v.) (leg. anonim., September 1988, 915, n.spec. =1); Portorož: Dragonja (v.), Dragonja (r.), mouth to Pinjevac (r.) (leg. B. Kryštufek 01/05/1986, 915, n.spec. =1); Dragonja (r.), Stena (leg. G. Džukić, B. Kryštufek 25/08/1982, 915, n.spec. = 1); (leg. BID “Josif Pančić” 22/08/1976, 915, n.spec. = 1).

\section{Podarcis tauricus}

Macedonia Southern Mountains Mariovo: Vitolište (v.), Dunje (v.) - Vitolište (v.), road (leg. G. Džukić, 28/05/2004, 115, n.spec. = 1); South-western Region Prespansko jezero Lake: Pretor (leg. A. Ćetković, 11/08/1990, 115, n.spec. =1); Vardar Zone Đevđelija: Stojakovo (v.) (leg. D. Pavićević, 02/05/1985, 115, n.spec. = 2); Gradsko: surrounding (leg. G. Džukić 15/04/1972, 115, n.spec. = 1); Katlanovo (leg. anonim. 24/04/1985, 115, n.spec. $=2$ ); Serbia Bačka Subotica: Mali Pijac (v.) (leg. J. Mikuška, 01/05/1963, 180, n.spec. = 4); Banat Deliblatska peščara: Deliblato (v.), Veliki put (leg. G. Džukić, 19/05/1969, 197, n.spec. = 4); (leg. anonim. 18/03/1979, 197, n.spec. = 1); Kajtasovo (v.) (leg. A. Ćetković 07/05/1995, 197, n.spec. = 2); Šumarak (v.), Majur bara pond (leg. G. Džukić 09/04/1981, 197, n.spec. = 1); Ružino polje (leg. anonim. 20/06/2008, 197, n.spec. =1); (leg. G. Džukić 18/05/1995, 197, n.spec. = 2); (leg. G. Džukić, M. Kalezić 18/05/1995, 197, n.spec. = 3); (leg. anonim. 03/05/1969, 197, n.spec. = 6); (leg. G. Džukić, M. Kalezić, M. Danoel 18/06/2007, 197, n.spec. = 1); Kosovo Gnjilane: city (leg. A. Ćetković, 25/09/1989, 195, n.spec. = 5); Ibar (r.) - valley: Lozište (v.), Košutovački potok, 14 km after Mitrovica (leg. P. Jakšić 28/05/1985, 196, n.spec. =1); Novo Brdo hill: Klobukar (v.) (leg. P. Jakšić 22/05/1985, 196, n.spec. = 2; 10/04/1986, 196); Metohia Prizren: Vrbnica (v.) (leg. G. Džukić, 07/07/1969, 179, n.spec. =4); Suva Reka: Mušutište (v.), Monastery Sveta Trojica (leg. G. Džukić 05/07/1979, 179, n.spec. =1); North-eastern Serbia Đerdapska klisura gorge: Cuce (leg. anonim., 23/07/1978, 194, n.spec. =2); Negotin: Radujevac 
(v.) (leg. G. Džukić 08/06/1972, 194, n.spec. = 3); Zamna - canyon (r.), Štubički Drenjar (leg. anonim. 21/04/1988, 194, n.spec. =4); South-eastern Serbia Trgovište: Crnovska Reka (r.) (leg. anonim., 18.06.-19.06.2006; 29.04.2000, 139, n.spec. =37); (leg. S. Đorđević 20/04/2007, 145, n.spec. $=$ 16); Southern Serbia Preševo: Orahovica hill (leg. G. Džukić, 13/05/1977, 193, n.spec. = 2); Slavujevac (v.) (leg. G. Džukić 17/05/1977, 193, n.spec. =7); Rujan Mt.: Biljača (v.), Pržar hill (leg. anonim. 14/05/1977, 193, n.spec. =2); Western Serbia Čemerno Mt.: Ibar - gorge (r.) (leg. P. Lazarević, 27/04/2002, 180, n.spec. =1).

\section{Zootoca vivipara}

S. I. (leg. anonim. s. d., 1589, n.spec. = 6); Bosnia \& Herzegovina Bosnia Treskavica Mt.: Veliko jezero Lake (leg. anonim., 10/06/1951, 73, n.spec. = 4); (leg. anonim. 12/08/1953, 73, n.spec. = 21); Croatia Lika Velebit Mt.: Jablanac (v.), Golić hill (leg. B. Jalžić, 16/08/1976, 70, n.spec. = 1); Montenegro Eastern Montenegro Bjelasica Mt.: Ćupovi (leg. G. Džukić, J. Crnobrnja Isailović, I. Aleksić, 29/07/1997, 75, n.spec. = 1); Lalevića Dolovi (leg. Lj. Tomović, R. Ajtić 25/06/2001, 75, n.spec. =6); Međedak (leg. G. Džukić, J. Crnobrnja Isailović, I. Aleksić 24/07/1997, 75, n.spec. =2; s. d., 75); Pešića jezero Lake (leg. G. Džukić, J. Crnobrnja 12/07/1996, 75, n.spec. =4); Prokletije Mts.: Maja Kolata (leg. anonim. 16/07/2011, 183, n.spec. =1); Ridsko jezero Lake, Hridski Krš (leg. G. Mesaroš 07/07/1990, 183, n.spec. = 1); Vusanje, Ćaf Bor (leg. G. Džukić 07/07/1995, 183, n.spec. = 1); Katun Čelića (leg. G. Džukić 07/07/1995, 183, n.spec. = 1); Russia Bashkirskaya ASSR Karaidelski Region: Bagazi (v.) (leg. L. M. Orlov, 20.05.$-10.06 .1973,141$, n.spec. = 2); Serbia Central Serbia Kopaonik Mt.: Gobelja (leg. A. Cetković, 15/07/1987, 71, n.spec. = 2); (leg. G. Džukić 29/06/1969, 71, n.spec. =1); Karaman (leg. G. Džukić 22/06/1971, 71, n.spec. = 1); Mala Gobelja (leg. G. Džukić 12/05/1976, 71, n.spec. = 3); Mali Karaman (leg. D. Jović 20/08/2009, 71, n.spec. = 7); Nebeske Stolice (leg. D. Jović 20/08/2009, 71, n.spec. = 1); Pančićev Vrh (leg. D. Jović 28/07/2007, 71, n.spec. =2); (leg. Lj. Tomović, R. Ajtić 23.08.$-25.08 .2000,72$, n.spec. = 18); Srebrnac (leg. G. Džukić, M. Kalezić 15/06/2001, 71, n.spec. =2); Suvo Rudište (leg. anonim. 26/04/1951, 71, n.spec. = 3); (leg. B. Petrov 20/07/1983, 71, n.spec. =1); (leg. $M$. Belanović 06/08/1977, 71, n.spec. = 1); Vučak (leg. S. Stamenković 06/06/1985, 71, n.spec. = 4); (leg. anonim. 19/08/1986, 74, n.spec. =1; 24/07/1987, 74; s. d., 74); (leg. Lj. Orlandić 05/06/1985, 74, n.spec. =1); Eastern Serbia Stara planina Mt.: Kopren (leg. anonim., 28/07/1962, 152, n.spec. =1); (leg. D. Jović, M. Kalezić 07/07/2007, 152, n.spec. = 2); (leg. D. Jović, N. Sekulić 08/05/2005, 152, n.spec. = 1); Stražna Čuka (leg. G. Džukić, M. Kalezić, D. Jović 02/07/2008, 152, n.spec. =10); Kosovo Šar planina Mts.: Dolovi, toward Jažinačko jezero Lake (leg. G. Mesaroš, 24/06/1995, 69, n.spec. =1); Piribeg, Durlov potok (leg. P. Jakšić 03/07/1988, 69, n.spec. = 2; 13/07/1988, 69; 13/08/1991, 69); Durlov potok, toward Jažinačko jezero Lake (leg. anonim. 09/06/1953, 69, n.spec. =2); Stojkova kuća (leg. G. Džukić 18/10/2008, 69, n.spec. = 1); (leg. V. Stevanović 02/07/1980, 69, n.spec. =1); toward the band, above Stojkova kuća (leg. G. Džukić 02/07/1980, 69, n.spec. =1); Štrpce, Štrbački Jalovarnik (leg. P. Jakšić 02/08/1988, 69, n.spec. = 3); (leg. anonim. 22/07/1949, 69, n.spec. =4); Metohia Mokra Gora Mt.: Mokra planina Mt., Berim (leg. G. Džukić, 10/06/1977, 182, n.spec. =1); Savine vode (leg. G. Džukić 08/06/1977, 182, n.spec. =2); Šar planina Mts.: Velibeg and Šutman, Čemerište (leg. G. Džukić, M. Kalezić, J. Crnobrnja Isailović 25.07.-28.07.1995, 181, n.spec. = 76); Western Serbia Tara planina Mt.: Zaovine (v.), Beli Rzav, lake (leg. G. Džukić, 20/05/1972, 182, n.spec. =3).

\section{PHRYNOSOMATIDAE}

\section{Phrynosoma cornutum}

USA California Camp Ventura (leg. Sanders, Baxter, 09/04/1948, 750, n.spec. $=1 ; 12 / 04$ / 1948, 750). 


\section{PHYLLODACTYLIDAE}

\section{Tarentola mauritanica}

Italy Salerno Capaccio Scalo: Pestum (leg. G. Džukić, 07.06.-22.06.1978, 192, n.spec. =4).

\section{SCINCIDAE}

\section{Ablepharus bivittatus}

Afghanistan Bamyan Bandi-I-Amir (leg. anonim., 04/08/1972, 779, n.spec. = 1; 05/08/ 1972, 779).

\section{Ablepharus kitaibelii}

Macedonia Central Mountains Jakupica Mt.: Papradište (v.), Bogomila (leg. G. Pasuljević, 1960, 62, n.spec. = 23); South-western Region Prespansko jezero Lake: Rajca (leg. G. Džukić, 24/05/2002, 56, n.spec. = 11); Vardar Zone Negotino: Krivolak (leg. A. Ćetković, 24/05/1989, 56, n.spec. $=2$ ); Montenegro Eastern Montenegro Bjelasica Mt.: Međedak (leg. M. Stanković, 22/08/1994, 54, n.spec. =1); Serbia Central Serbia Kopaonik Mt.: Jošanička Banja (leg. J. Crnobrnja Isailović, 02/06/1986, 57, n.spec. =1); Krmeljica (leg. A. Cetković 23/05/2002, 57, n.spec. = 1); Kuršumlija: Kosovska Rača (v.) (leg. Milenković 1976, 57, n.spec. $=1$ ); Rudare (v.), Rudare (v.) - Prolom Banja, road (leg. Lj. Tomović, K. Ljubisavljević 02.05. \& 19.06.1998, 57, n.spec. = 11); Prokuplje: Viča (v.) (leg. Lj. Tomović 14.06. \& 15.06.1999, 57, n.spec. =21); Vrnjačka Banja (leg. G. Pasuljević 1960, 57, n.spec. =6); Eastern Serbia Sićevačka klisura gorge: Crveni Breg (v.), Krst hill (leg. B. Žiljak, 10/06/1997, 63, n.spec. =2); Soko Banja (leg. G. Pasuljević 1960, 63 , n.spec. $=25$ ); Kosovo Obilić (leg. G. Pasuljević, 01/04/1967, 58, n.spec. =1); Podujevo: Velika Reka (v.) (leg. G. Pasuljević 1960, 64, n.spec. = 38); Priština: Gračaničko jezero Lake (leg. G. Pasuljević 1960, 65, n.spec. =1); Grmija hill (leg. G. Pasuljević 1960, 59, n.spec. = 96; s. d., 58); (leg. P. Jakšić 10/06/1991, 58, n.spec. =1); Kišnica (v.) (leg. G. Pasuljević 1960, 65, n.spec. = 5); (leg. G. Pasuljević 1960, 65, n.spec. =20); Metohia Istok: Osojane (v.) (leg. G. Pasuljević, 1960, 67, n.spec. =2); Peć (leg. G. Pasuljević 1960, 67, n.spec. =7); Suva Reka: Samodraža (v.) (leg. G. Pasuljević 1960, 67, n.spec. = 1); North-eastern Serbia Kladovo: Manastirica (v.), Podvrška, Pristav (leg. G. Džukić, 20/06/2003, 55, n.spec. = 1); Velesnica (v.) (leg. J. Crnobrnja Isailović, I. Aleksić 30/05/1998, 55, n.spec. $=10$ ); Negotin: Jabukovac (v.), Zamna (r.), Balta Ursuluj (leg. G. Džukić, M. Kalezić 28/09/2005, 55, n.spec. = 2); Plavna (v.), Zamna (r.) - canyon (leg. G. Džukić 21/04/1988, 55, n.spec. = 2); Paraćin (leg. J. Mikuška 1967, 55, n.spec. $=1$ ); North-western Serbia Donji Milanovac: Mosna (v.) (leg. G. Pasuljević, 1960, 66, n.spec. =10); Suvobor Mt.: Ravna Gora, Nikolića Koliba (leg. M. Plećaš 24/04/2004, 66, n.spec. = 1); Valjevo: Gradac (r.) - canyon, 7 km south-western from Valjevo (leg. A. Glišić 04/03/2006, 66, n.spec. =1); Pomoravlje Ćuprija: Senje (v.), Monastery Ravanica (leg. G. Džukić, M. Kalezić, 14/05/2000, 55, n.spec. = 3); South-eastern Serbia Trgovište: Crnovska Reka (r.) (leg. Lj. Tomović, R. Ajtić, Easter 2004, 68, n.spec. =9); Southern Serbia Rujan Mt.: Biljača (v.), Pržar hill (leg. G. Džukić, 14/05/1977, 68, n.spec. $=2$ ); Srem Fruška Gora Mt.: Testera (leg. G. Pasuljević, 1960, 61, n.spec. = 28); (leg. M. Stanković 1992, 61, n.spec. = 1); Šumadija Avala Mt.: Trešnja (leg. I. Petrov, 04/07/1996, 60, n.spec. =1); (leg. anonim. 17/10/1948, 60, n.spec. =1); Beograd: Čukarica, Košutnjak (leg. G. Pasuljević 1960, 60, n.spec. $=80$ ); Western Serbia Užice: city, surrounding of old city (leg. M. Gvozdić, 29/04/1992, 66, n.spec. =1).

\section{Chalcides ocellatus}

Greece Crete (i.) Festos port (leg. Lj., S. Marinković, 29/06/1991, 154, n.spec. = 1); Morocco (leg. anonim. 1959, 154, n.spec. =1). 


\title{
Eurylepis taeniolata \\ Afghanistan Samangan Takht-e-Rostam (leg. anonim., 16/08/1972, 168, n.spec. = 1).
}

\author{
Eutropis dissimilis \\ Afghanistan Nangarhar Džalalabad: Hada (leg. anonim., 25/08/1972, 169, n.spec. =1).
}

\section{Serpentes}

\section{COLUBRIDAE}

\section{Boiga blandingii}

Liberia Monrovia Suakoko (leg. anonim., s. d., 1904, n.spec. =1).

\section{Coronella austriaca}

S. I. (leg. anonim. s. d., 1909, n.spec. $=7$ ); Croatia Central Dalmatia Biokovo Mt.: new road to Vrgorac (v.) (leg. N. Tvrtković, 30/08/1976, 925, n.spec. = 1); Lika Plješivica: Mihaljevac (v.) (leg. A. Vlajić, 02/07/1986, 925, n.spec. =1); Southern Dalmatia Mljet (i.): Babine Kuće (v.) Pomene (v.) (leg. B. Kral, 18/08/1977, 925, n.spec. =1); Zagorje Samobor: Oštrc (leg. T. Rađa, 08/05/1976, 925, n.spec. = 1); Macedonia Southern Mountains Kožuf Mt.: Smrdlivi Vodi (leg. anonim., 09/07/1990, 448, n.spec. =1); South-western Region Pelister Mt.: Debeli Rid (leg. A. Vlajić, 04/06/1988, 448, n.spec. = 1); Western Mountains Šar planina Mts. (leg. anonim., 01/08/1954, 448, n.spec. = 1); Montenegro Central Montenegro Gornja Morača: Sirovac (leg. G. Džukić, J. Crnobrnja, I. Aleksić, 30/07/1997, 510, n.spec. =1); Eastern Montenegro Berane: Rujišta (v.) (leg. anonim., 30/05/2003, 509, n.spec. =1); Bijelo Polje: Čokrlije (v.) (leg. T. Tomović 04/07/1989, 509, n.spec. =1); Prokletije Mts.: Grbaja valley, Ljubokuće (leg. R. Mandić 03/07/1984, 509, n.spec. =1); Gusinje, toward Grbaja mountain house (leg. G. Džukić 03/07/1984, 509, n.spec. = 1); Northern Montenegro Durmitor Mt.: Komarnica (r.), Komarnica (v.) (leg. S. Marinković, s. d., 418, n.spec. = 1); Nevidio canyon, bellow Žute Grede (leg. G. Džukić 22/08/1984, 418, n.spec. = 1); Virak (v.), Smrčevo Brdo hill (leg. D. Pavićević 26/06/1987, 418, n.spec. =1); Tara (r.) - canyon: Aluge - Rasove (leg. G. Džukić, M. Kalezić, M. Marković 03/06/2003, 939, n.spec. = 1); Bajlovića Sige (leg. D. Bejaković 21.08.-27.08.1988, 946, n.spec. = 1); Đurđevića Tara, Splavište (leg. P. Jakšić 24/06/1985, 946, n.spec. = 1); Tepca (v.), Devojački Most (leg. M. Langurov 13/07/2001, 939, n.spec. = 3); (leg. anonim. July 2005, 939, n.spec. = 1); (leg. G. Džukić 04/07/1988, 946, n.spec. = 4); Submediterranean Montenegro Orjen Mt.: Mala Siljevica (leg. M. Milenković, 02/06/1979, 510, n.spec. =1); Podgorica: $2 \mathrm{~km}$ toward Bioče (v.) (leg. G. Džukić, M. Kalezić s. d., 510, n.spec. =1); Western Montenegro Nikšić: Vilusi (v.), Gornja Spila (v.) - Donja Spila (v.) (leg. G. Džukić, 05/06/1984, 510, n.spec. =1); Serbia Banat Bela Crkva: Kusić (v.) (leg. anonim., 17/09/2006, 944, n.spec. = 1); Deliblatska peščara: Dolina (leg. Z. Gradojević 25/06/1954, 944, n.spec. =1); Dubovac (cottage) (leg. G. Džukić 23/06/1994, 944, n.spec. = 1); (leg. M. Čobanov 1969, 944, n.spec. =2); Kovin: Crna Bara pond, Rimski šanac (leg. G. Džukić 17/06/1979, 944, n.spec. =1); Vršac: Jablanka (v.), crossroad v. Jablanka (v.) - Mesić (v.) (leg. G. Džukić, M. Kalezić, M. Marković 19/10/2002, 944, n.spec. = 1); Vršački Breg Mt.: Jablanka (v.), Vojpotok (leg. L. Novak 18/06/2004, 945, n.spec. =1); Mesić (v.), Mesićki potok, Tri bunara (leg. Đ. Novak 10/07/2004, 945, n.spec. =1); Sočica (r.) (leg. anonim. 01/04/2003, 945, n.spec. =1); Široko Bilo, mountain house (leg. anonim. 19/06/1969, 945, n.spec. =1); (leg. Đ. Novak 14/06/2004, 945, n.spec. $=1$ ); toward Lisičji vrh peak (leg. P. Petrović 17/05/1976, 945, n.spec. = 1); (leg. anonim. 25/08/1991, 453, n.spec. = 5); (leg. G. Džukić 14/07/1991, 453, n.spec. = 1); (leg. Đ. Novak, L. Novak 15/06/2005, 945, n.spec. = 1); Central Serbia Jastrebac Mt.: Buci (v.) (leg. G. Džukić, 23/04/1985, 522, n.spec. =1); Kopaonik Mt.: Gobelja (leg. Lj. Janković July 2002, 522, n.spec. = 1); Lisina (v.) (leg. anonim. 24/04/1952, 522, n.spec. = 1); Suvo Rudište (leg. anonim. 24/04/1952, 522, n.spec. =1); Kraljevo: Sirča (v.) (leg. $P$. 
Marinković 08/08/1991, 522, n.spec. =1); Eastern Serbia Knjaževac: Stanjinac (v.), $2 \mathrm{~km}$ from v. Kalna (leg. G. Džukić, M. Kalezić, 14/06/2006, 446, n.spec. = 1); Pirot: Jerma (r.) - gorge (leg. Lj. Tomović 02/05/2002, 446, n.spec. =1); Stara planina Mt.: Topli Do (v.), Toplodolske livade (leg. $P$. Jakšić 14/07/1991, 446, n.spec. = 1); Stol Mt. (leg. M. Belanović 23/05/1978, 446, n.spec. = 1); Kosovo Novo Brdo hill: Bostane (v.) (leg. P. Jakšić, 12/04/1986, 463, n.spec. =1); Čuljkovci (v.) (leg. P. Jakšić 21/05/1986, 445, n.spec. = 1); Izvor (v.) (leg. P. Jakšić 22/07/1985, 463, n.spec. = 1; 25/05/1986, 463); Klobukar (v.) (leg. P. Jakšić 15/05/1985, 445, n.spec. =1); Makreš (v.) (leg. $P$. Jakšić 10/04/1986, 463, n.spec. = 1); Manišince (v.) (leg. P. Jakšić 22/05/1985, 445, n.spec. = 2); Prekovce (v.), Tanki Zabelj (v.) (leg. P. Jakšić 13/05/1986, 463, n.spec. = 1); (leg. P. Jakšić 10/04/1986, 445, n.spec. = 2); Prilepnica (v.), Jasenovik (v.) (leg. P. Jakšić 15/05/1985, 445, n.spec. $=$ 1); Straševina (v.) (leg. P. Jakšić 24/09/1986, 463, n.spec. = 1); Šumaci (v.) (leg. P. Jakšić 21/05/1986, 463, n.spec. = 1); Šar planina Mts.: Ošljak Mt., peak (leg. P. Jakšić 20/08/1989, 461, n.spec. = 1); Piribeg Mt. (leg. P. Jakšić 20/07/1986, 461, n.spec. =1); Metohia Prokletije Mts.: Dečani - Peć (leg. Lj. Janković, 12/07/1976, 461, n.spec. =1); North-eastern Serbia Kučajske planine Mts.: Grza (r.), cottage (leg. I. Pavković, 13/07/2009, 928, n.spec. = 1); Malinik Mt.: Lazareva Reka (r.) - canyon, between Sovina Glava and Vernikica (leg. G. Džukić 01/06/1983, 928, n.spec. = 1); Zlot (v.), above Lazareva pećina cave (leg. S. Marinković 10/07/1982, 928, n.spec. = 1); Negotin: Klokočevac (v.) - Plavna (v.) (leg. G. Džukić 31/05/1986, 928, n.spec. = 1); North-western Serbia Donji Milanovac: Liškovac (leg. D. Pavićević, 01/05/1987, 943, n.spec. =1); Ljubovija: Gornje Košlje (v.) (leg. S. Marinković 21/05/1986, 943, n.spec. =1); Uzovnica (v.) (leg. anonim. 07/07/1977, 943, n.spec. =1); Maljen Mt.: Divčibare, Skakavac, road to Crni Vrh peak (leg. N. Milenković 05/08/1991, 943, n.spec. = 1); Stari Grad, tourist colony (leg. D. Đurić 27/08/1988, 943, n.spec. =1); (leg. I. Knežević s. d., 943, n.spec. =1); Povlen Mt.: Debelo Brdo hill (leg. anonim. s. d., 943, n.spec. $=1$ ); South-eastern Serbia Crna Trava: Brod (v.) (leg. G. Džukić, M. Kalezić, 23/06/2007, 446, n.spec. = 1); South-western Serbia Jadovnik Mt.: Milošev Do (v.), Prosoje (leg. B. Jovanović, B. llić, 28/07/2013, 461, n.spec. =1); Mokra Gora Mt.: Ribariće (v.), Monastery Crna Reka (leg. G. Džukić 11/06/1977, 461, n.spec. =1); Srem Fruška Gora Mt.: Andrevlje (leg. G. Džukić, 25/05/1979, 527, n.spec. = 1); Šumadija Beograd: surroundings (leg. S. Marinković, 1988, 527, n.spec. =1); Bukulja Mt. (leg. anonim. 1954, 527, n.spec. =1); Gornji Milanovac: Beršići (v.) (leg. D. Kataranovski 01/05/1996, 527, n.spec. $=1$ ); Western Serbia Nova Varoš: Zlatasko jezero Lake, Vasilići (h.) (leg. B. Zorić, 18/09/2005, 941, n.spec. = 1); Požega: Zdravčići (v.) (leg. anonim. s. d., 941, n.spec. = 1); Tara planina Mt.: Batura, Đurđevo brdo hill (leg. anonim. 11/06/1969, 941, n.spec. = 2); Predov Krst - Brusnica, Galinska Reka (r.) (leg. anonim. 12/06/1969, 941, n.spec. =1); Zlatibor Mt.: Čavlovac (leg. M. Belanović 11/07/1972, 941, n.spec. =1); Gaj (v.) (leg. M. Gvozdić 31/05/1992, 941, n.spec. =1); Šargan (leg. I. Karaman, B. Talošin 14/06/1991, 941, n.spec. =1).

\section{Dolichophis caspius}

S. I. (leg. anonim. s. d., 1910, n.spec. =6); Macedonia Central Mountains Babuna Mt.: Derven (leg. G. Džukić, 10/05/1972, 508, n.spec. = 2); South-western Region Prespansko jezero Lake: Pretor (v.) (leg. G. Mesaroš, s. d., 508, n.spec. =1); Montenegro Mediterranean Montenegro Ulcinj: Vada (i.) (leg. anonim., 02/04/1989, 440, n.spec. =1); Romania Moldova Noua Požežena: Šuška, at watchtower (leg. G. Džukić, 07/09/2013, 440, n.spec. =1); Serbia Banat Bela Crkva: Crvena Crkva (v.), before the village (leg. G. Džukić, 02/06/2014, 375, n.spec. $=$ 1); Dupljaja (v.) - Grebenac (v.), Svinjska plaža (leg. V. Kalafatić, M. Tanasković 26/10/2007, 374, n.spec. =1); Vračev Gaj (v.), Kremzerov Salaš (leg. G. Džukić s. d., 375, n.spec. =1); Deliblatska peščara: Kajtasovo (v.), Mali Pesak (leg. M. Marković November 1987, 374, n.spec. = 1); Šumarak (v.) (leg. G. Džukić 16/08/2004, 375, n.spec. = 1); Šušara (v.), Čardak (leg. G. Džukić 19/05/1969, 374, n.spec. =1); Kovin: city (leg. G. Džukić 26/04/1969, 375, n.spec. =1); Rašće (leg. anonim. 02/10/2010, 374, n.spec. =1); Central Serbia Kopaonik Mt.: Jošanička Banja, exit (leg. anonim., 18/07/1987, 432, n.spec. =1); Leskovac: Belanovce (v.) (leg. S. Antić s. d., 432, n.spec. =1); Eastern Serbia Niš: Palilula, Ledena stena, toward Novo Selo (v.) (leg. D. Jović, 30/06/2008, 376, n.spec. $=1$ ); Pirot: Gornja Kamenica (v.), $2 \mathrm{~km}$ before the village, toward Pirot (leg. anonim. 11/07/1989, 376, n.spec. = 2); Rtanj Mt.: Moravica (r.) - gorge (leg. D. Jović 24/06/2008, 376, 
n.spec. = 1); North-eastern Serbia Kladovo: Vajuga (v.) (leg. G. Džukić, M. Kalezić, 20/06/2007, 417 , n.spec. =1); Negotin: Konjušnica (v.) (leg. anonim. March 2006, 417, n.spec. =1); (leg. G. Džukić, M. Kalezić 21/06/2003, 417, n.spec. =1); Pomoravlje Golubac: Vinci (v.), exit toward Usije (v.) (leg. G. Džukić, 28/05/2011, 417, n.spec. =1); South-eastern Serbia Starac Mt.: Starac (v.), Gornji Starac (leg. Lj. Tomović, R. Ajtić, 05/05/2002, 377, n.spec. =3); Southern Serbia Bojnik: Crkvice (v.) (leg. G. Džukić, M. Kalezić, 15/06/2001, 377, n.spec. =1); Srem Fruška Gora Mt.: Čortanovci (v.) (leg. M. Mikeš, J. Šoti, 30/05/1978, 430, n.spec. = 1); Koševac, Podčenta (leg. G. Džukić 10/07/1974, 430, n.spec. = 1); Šumadija Avala Mt.: Vrčin (v.), Brđani, Džombina rupa (leg. M. Kovačević, 10/11/1985, 429, n.spec. =1); (leg. anonim. 17/10/1978, 429, n.spec. =1); Beograd: Čukarica, Košutnjak (leg. anonim. 20/04/1960, 420, n.spec. =1); Košutnjak, quarry (leg. G. Džukić 09/05/1969, 420, n.spec. =1); Palilula, Lešće cemetery (leg. anonim. 23/05/2000, 420, n.spec. $=1$ ); Savski Venac, Topčider, Dušan Radenković street no. 7 (leg. M. Milenković, S. Sekicki 26/06/2004, 420, n.spec. =1); Smederevska Palanka: Kusadak (v.) (leg. anonim. 1957, 429, n.spec. =1).

\section{Elaphe quatuorlineata}

Macedonia Vardar Zone Katlanovo: Divle (v.), Raklje (leg. G. Džukić, M. Belanović, spring-summer 1978, 408, n.spec. = 1); Montenegro Mediterranean Montenegro Ulcinj: Šaško jezero Lake (leg. anonim., 12/07/1969, 381, n.spec. $=1$ ); Submediterranean Montenegro Orjen Mt.: Ledenice (leg. G. Džukić, 04/06/1984, 381, n.spec. = 1); Skadarsko jezero Lake: Godinje (v.) (leg. G. Džukić, M. Kalezić 10/06/2008, 381, n.spec. = 1); Murići (v.), beach (leg. anonim. s. d., 381, n.spec. =1); Virpazar, Besac, toward Limljani (v.) (leg. G. Džukić, M. Kalezić, M. Marković $31 / 05 / 2003,381$, n.spec. $=1)$.

\section{Hierophis gemonensis}

S. I. (leg. anonim. s. d., 1911, n.spec. =4); Bosnia \& Herzegovina Herzegovina Bileća: Donja Meka Gruda (v.) (leg. G. Džukić, 14/05/1983, 385, n.spec. =1); Mostar: Blagaj, Busak dry canyon (leg. S. Marinković 04/05/1986, 385, n.spec. =1); Popovo Polje: Strujići (v.) (leg. S. Marinković 02/07/1987, 385, n.spec. =1); Stolac - v. Predolje: $5 \mathrm{~km}$ before v. Predolje (leg. G. Džukić 15/05/1983, 385, n.spec. = 1); Trebinje: Lastva (v.), $4 \mathrm{~km}$ from Lastva (v.) toward Trebinje (leg. G. Džukić 11/06/1980, 385, n.spec. =1); Croatia Central Dalmatia Vis (i.): Pelegrin, Stončica (leg. S. Regner, March 1969, 434, n.spec. = 1); Istria Rovinj (leg. G. Džukić, 11/04/1969, 434, n.spec. = 1); Kvarner Cres (i.): Porozina (leg. N. Tvrtković, 17/08/1977, 434, n.spec. =1); Northern Dalmatia Kornati: Kornat (i.) (leg. M. Radovanović, 27/08/1956, 530, n.spec. = 1); Zadar: Bokanjac (leg. M. Radovanović July 1960, 427, n.spec. = 1); (leg. V. Pavlović 1958, 427, n.spec. = 2); Petrčane (v.), Punta Radman (leg. V. Stevanović 23/08/1983, 427, n.spec. =1); Southern Dalmatia Dubrovnik: Lokrum (i.) (accross) (leg. G. Džukić, 20/08/1981, 530, n.spec. =1); Zagora Obrovac: Kolašac (v.) (leg. G. Džukić, 31/05/1985, 530, n.spec. = 1); Macedonia South-western Region Debar: Debarska Banja (leg. S. Petkovski, V. Sidorovska, 24/08/1999, 402, n.spec. =2); Montenegro Mediterranean Montenegro Bar: Ostros (v.), exit from Ostros (v.) toward Bar (leg. anonim., 23/06/2004, 405, n.spec. =1); Budva: Bečići, hotel "Splendid" (leg. S. Marinković 02/10/1986, 405, n.spec. =1); Submediterranean Montenegro Danilovgrad: Garač Mt., Vodine (leg. G. Džukić, M. Kalezić, 29/05/2003, 405, n.spec. =1); Podgorica: Bolesestra (v.), Jelin Dub (leg. G. Džukić, M. Kalezić, M. Marković 03/06/2005, 405, n.spec. =1); Skadarsko jezero Lake: Gornji Murići (v.) (leg. anonim. 30/05/1995, 405, n.spec. =1); Murići (v.), road to Đuravci (v.) (leg. G. Džukić, J. Crnobrnja, I. Aleksić 30/05/1996, 405, n.spec. =1); Western Montenegro Nikšić: Vilusi (v.), road to Cerova Lokva pond (leg. G. Džukić, M. Kalezić, M. Marković, 26/06/2004, 405, n.spec. =1).

\section{Hierophis viridiflavus}

Croatia Istria Rovinj (leg. G. Džukić, 11/04/1969, 511, n.spec. = 1); Kvarner Krk (i.): road bend Punat - Baška (leg. G. Džukić, 26/04/1981, 511, n.spec. = 1). 


\section{Natrix natrix}

s. I. (leg. anonim. s. d., 1912, n.spec. = 21); Bosnia \& Herzegovina Bosnia Glamočko Polje: Karaizovci (v.) (leg. G. Džukić, 18/05/1983, 384, n.spec. = 2); Livanjsko Polje: Crni Lug (v.), Pržine (leg. G. Džukić 18/05/1983, 384, n.spec. = 5); Ždralovac (leg. N. Tvrtković 11/06/1977, 384, n.spec. = 2); Tuzla: Donje Dubrave (leg. G. Džukić 06/06/1985, 384, n.spec. =1); Herzegovina Mostar: Blagaj, Svinjarski Do (Gnojnice) (leg. S. Marinković, 03/07/1987, 384, n.spec. =1); Nevesinje - Boračko jezero Lake: Čitluk (v.) (leg. G. Džukić 16/05/1983, 384, n.spec. = 1); Croatia Baranja Kneževi Vinogradi: Baranjski Breg (leg. J. Mikuška, 09/04/1972, 460, n.spec. = 1); Kopački rit: Bilje, Biljski rit marsh (leg. J. Mikuška 07/04/1971, 460, n.spec. =1); Ćošak (leg. J. Mikuška 01/04/1971, 460, n.spec. $=1$; 08/04/1972, 460); Tikveš: Oršoš (leg. J. Mikuška 08/04/1972, 460, n.spec. = 1); Central Dalmatia Biokovo Mt.: new road to Vrgorac (v.) (leg. N. Tvrtković, 30/08/1976, 443, n.spec. =1); Gorski Kotar Delnice: Crni Lug (v.), Vela Voda (leg. D. Rudsner, 23/08/1963, 443, n.spec. = 1); Istria Labin: Salakovci (v.), Labin - Koromačno, road (leg. G. Džukić, 27/04/1981, 443, n.spec. = 2); Northern Dalmatia Zadar: Bokanjac (leg. M. Radovanović, s. d., 443, n.spec. =1); Posavina Sisak (leg. J. Mikuška, spring 1963, 443, n.spec. =1); Southern Dalmatia Metković: at Prevlaka (Meteriz) (leg. G. Džukić, 07/04/1989, 443, n.spec. =1); Macedonia Eastern Mountains Kratovo: Kratovska Reka (r.) - canyon, cave (leg. G. Džukić, 12/08/1982, 452 , n.spec. = 1); Vardar Zone Demir Kapija: Iberlijska klisura gorge (leg. P. Jakšić, 20/05/1988, 452, n.spec. =1); Katlanovo: Divle (v.), Raklje (leg. G. Džukić, M. Belanović spring-summer 1978, 452, n.spec. = 1); Negotino: Perza, Dubovo fishpond (leg. G. Džukić 25/06/1998, 452, n.spec. =1); Montenegro Eastern Montenegro Bjelasica Mt.: Biogradsko jezero Lake (leg. Lj. Tomović, R. Ajtić, 18/07/2000, 523, n.spec. = 2); Lalevića Dolovi (leg. R. Ajtić 2002, 523, n.spec. = 1); mountain house (leg. J. Crnobrnja Isailović 13/07/1996, 523, n.spec. = 1); Prokletije Mts.: Plav, Plavsko jezero Lake (leg. Z. Franolić, J. Crnobrnja, D. Čobić 03/07/1984, 523, n.spec. = 1); Mediterranean Montenegro Budva: Jaz (r.), $1 \mathrm{~km}$ up from the mouth (leg. A. Hegediš, 01/08/1990, 926, n.spec. =1); Mrčevo Polje (leg. anonim. 27/05/1996, 926, n.spec. =1); Ulcinj: Donji Štoj (v.), school Bregvija (leg. G. Džukić, M. Kalezić, M. Marković 23/06/2004, 926, n.spec. = 2); Šaško jezero Lake (leg. anonim. 12/07/1969, 926, n.spec. =1); Northern Montenegro Durmitor Mt.: Sedlo (bellow), road to Lojanik, road to Lojanik (leg. P. Jakšić, 03/07/1987, 516, n.spec. =1); Škrčko Ždrijelo, bellow (leg. G. Džukić 23/08/1984, 516, n.spec. = 1); Mojkovac: Tara (r.) (leg. P. Jakšić 10/08/1986, 516, n.spec. =1); Sinjajevina Mt.: Kučajevica, Zminičko jezero Lake (leg. G. Džukić 10/07/1971, 516, n.spec. = 1); Tara (r.) - canyon: Budečevica (leg. G. Džukić 12/07/1971, 516, n.spec. = 1); Submediterranean Montenegro Lovćen Mt.: Ivanova Korita (leg. M. Radovanović, 28/07/1959, 519 , n.spec. = 1); Lovćensko jezero Lake (leg. G. Džukić 02/06/1984, 519, n.spec. =1); Orjen Mt.: Kruševice (v.), Donji Lokanj (leg. G. Džukić, M. Kalezić 24/08/2001, 519, n.spec. = 1); Podgorica: Lijeva Rijeka (leg. anonim. 17/09/1990, 519, n.spec. =1); Skadarsko jezero Lake: Orahovačko Polje (leg. G. Džukić 09/04/1992, 519, n.spec. =1); Western Montenegro Nikšić: Glavice (v.), spring (leg. G. Džukić, M. Kalezić, 11/06/2005, 947, n.spec. = 1); Grahovac (v.), Kotlić (leg. G. Džukić, M. Kalezić, M. Marković 25/06/2004, 947, n.spec. =1); Spila (v.), Voluje oko (leg. G. Džukić, M. Kalezić 25/06/2004, 947, n.spec. = 2); Velika Osječenica (v.) (leg. G. Džukić 24.08.-27.08.1982, 947, n.spec. =4); (leg. G. Džukić, M. Kalezić 25/06/2004, 947, n.spec. = 1); Vilusi (v.), Kovačevića Lokva pond (leg. G. Džukić, M. Kalezić, M. Marković 25/06/2004, 947, n.spec. =1); Plužine: Bajovo Polje (v.), Bratuljev Do, Potrk (leg. G. Džukić, M. Kalezić 07/06/2008, 947, n.spec. =1); Serbia Bačka Subotica: Palić (v.), Mali Pijac (v.) (leg. J. Mikuška, 01/05/1963, 389, n.spec. = 3); Banat Alibunar: Janošik (v.), toward Alibunar (leg. anonim., 24/10/2004, 383, n.spec. =1); Bela Crkva: Crvena Crkva (v.) (leg. M. Marković s. d., 923, n.spec. = 1); Mrtva Nera (leg. G. Džukić, M. Kalezić 05/04/2003, 923, n.spec. = 1); Beograd: Palilula, Krnjača, fishpond "Mika Alas" (leg. G. Džukić 07/09/1973, 395, n.spec. $=2$ ); Pančevački rit marsh, Krnjača, fishpond "Mika Alas" (leg. S. Marinković 14/09/1986, 395, n.spec. $=1$ ); Deliblatska peščara: Deliblato (v.), railroad to Mramorak (v.) (leg. G. Džukić 16/08/2004, 451, n.spec. = 1); Flamunda (leg. V. Stevanović 14/07/1970, 469, n.spec. = 1); Gaj (v.), peat bog bellow Hatarica (leg. G. Džukić, M. Kalezić, A. Ivanović, M. Slijepčević, A. Urošević 10/06/2012, 469, n.spec. =1); Jasenovo (v.), Mlin, Šlajz (leg. G. Džukić, M. Kalezić 08/09/1998, 451, n.spec. = 1); Kajtasovo (v.), Đurica pond (leg. G. Džukić, M. Kalezić, M. Marković 21/05/1998, 469, n.spec. =1); (leg. anonim. 12/10/2004, 451, n.spec. =1); Labudovo okno (leg. G. Džukić 01/05/2014, 469, n.spec. = 1); Majur bara pond (leg. G. Džukić 16/05/1976, 451, n.spec. =1); Stara Palanka (v.), between Đavolji most and v. Stara Palanka, canal DTD (leg. M. Munćan 1988, 469, n.spec. =1); Šušara (v.), Church Sveta Marija u pesku (leg. G. Džukić 21/04/1973, 451, n.spec. 
= 1); Utrine, Salaš Vlaških (leg. G. Džukić, M. Kalezić 26/06/1999, 469, n.spec. = 1); Stevanove ravnice (leg. G. Džukić 07/07/2014, 469, n.spec. = 1); Kovin: Crna Bara pond (leg. G. Džukić 24/05/1959, 395, n.spec. = 1); Idrifor, Dunav (r.) (leg. anonim. 28/07/1974, 395, n.spec. = 1); Pančevo: Ivanovo (v.) (leg. G. Džukić 02/04/1980, 395, n.spec. =1); Plandište: Banatski Sokolac (v.) (leg. G. Džukić, M. Kalezić 17/05/2003, 383, n.spec. = 1); Markovićevo (v.) (leg. G. Džukić, M. Kalezić 17/05/2003, 383, n.spec. = 1); Vršac: city (leg. P. Sučević July 2002, 923, n.spec. = 1); Veliko Središte (v.), near "Ekonomija" (leg. G. Džukić 10/06/2004, 923, n.spec. = 1); Vršački Breg Mt.: Široko Bilo, toward Gudurički vrh peak (leg. P. Petrović 17/05/1976, 923, n.spec. =2); Zrenjanin: Aradac (v.) (leg. Z. Dunđerski 23/10/1980, 395, n.spec. =1); Central Serbia Ćićevac: surroundings (leg. S. Mićić, 1959, 922, n.spec. = 3); Kraljevo: Sirča (v.), Zapadna Morava (r.) (leg. S. Marinković 01/06/1991, 922, n.spec. =1); Prokuplje: 15 km south-western (leg. anonim. 16/08/1961, 922, n.spec. =1); 2-3 km eastern (leg. anonim. 30/07/1961, 922, n.spec. =1); Eastern Serbia Sićevačka klisura gorge: Crvena Reka (v.) (leg. anonim., 26/09/2005, 447, n.spec. = 1); Ostrovica (v.), Banjica, Ždrelo (leg. Lj. Tomović 08/07/1997, 447, n.spec. = 1); Kosovo Kosovska Kamenica: Ograđe (v.) (leg. B. Milošević, 03/05/1978, 454, n.spec. = 1); Leposavić (leg. R. Kostović 01.05.-15.05.1975, 454, n.spec. = 1); Novo Brdo hill: Bostane (v.) (leg. P. Jakšić 12/04/1986, 518, n.spec. = 1; 25/05/1986, 456); Izvor (v.), Kriva Reka (r.) (leg. P. Jakšić 25/05/1986, 518, n.spec. =1); Ševa livada (leg. R. Lazić 13/05/1986, 456, n.spec. =1); Klobukar (v.) (leg. P. Jakšić 15/05/1985, 454, n.spec. = 3); Prekovce (v.), Zebince (v.) (leg. P. Jakšić 19/05/1986, 518, n.spec. = 1); (leg. P. Jakšić 05/06/1985, 456, n.spec. = 1); Trnićevce (v.) (leg. P. Jakšić May 1985, 454, n.spec. = 1); Vrkljaga (v.) (leg. D. Trajković 11/05/1986, 456, n.spec. = 1); (leg. P. Jakšić 05/05/1986, 518, n.spec. = 1); Šar planina Mts.: Brezovica, Piljevački potok (leg. P. Jakšić 06/08/1985, 454, n.spec. = 1); (leg. G. Pasuljević s. d., 454, n.spec. = 2); North-eastern Serbia Beljanica Mt.: Buk (leg. G. Džukić, 02/06/1979, 512, n.spec. = 1); Resava (r.), between the mountain house and motel "Lisina" (leg. B. Žiljak middle of May 1995, 512, n.spec. = 1); Kučajske planine Mts.: Grza (r.), Veliko jezero Lake (leg. U. Buzurović 14/07/2009, 512, n.spec. = 1); Grza (r.) - gorge (leg. D. Pavićević 19/07/1966, 512, n.spec. = 1); Majdanpek: Debeli Lug (v.) (leg. K. Vasić 15/05/1989, 512, n.spec. =1); Malinik Mt.: Lazareva Reka (r.) - canyon (leg. G. Džukić 09/07/1982, 512, n.spec. =2); Miroč Mt.: Vladimir Kiljež pond (leg. G. Džukić, M. Kalezić 14/05/2002, 512, n.spec. =1); Negotin (leg. D. Pavićević 18/06/1971, 512, n.spec. = 1); North-western Serbia Koceljeva: Kamenica (v.), Subotica (v.) - Kamenica (v.) (leg. G. Džukić, M. Kalezić, 25/04/1998, 394, n.spec. = 1); Loznica: Lešnica (v.) (leg. anonim. April 1990, 394, n.spec. = 1); Ljubovija: Tribuća (r.) - canyon (leg. anonim. 05/07/1978, 394, n.spec. = 1); Maljen Mt.: Divčibare, Rastovac (leg. I. Knežević 08/09/1996, 394, n.spec. = 1); Mionica: Gornji Mušić (v.) (leg. G. Džukić 29/05/1980, 394, n.spec. =1); Vladimirci: Vlasanica (v.), Mićina bara pond (leg. anonim. 16/05/1993, 394, n.spec. =1); Pomoravlje Ćuprija: Virine (v.) (leg. G. Džukić, M. Kalezić, 12/05/2002, 401, n.spec. = 1); Paraćin: Izvor (v.), Grza, Veliko jezero Lake (r.) (leg. S. Đurović 12/07/2010, 401, n.spec. = 1); Petrovac na Mlavi: Orljevo (v.) (leg. M. Milenković 23/10/1994, 401, n.spec. = 1); Smederevo: city, near the tower, Dunav (r.) (leg. M. Gvozdić 08/09/1992, 401, n.spec. =1); Veliko Gradište: Srebrno jezero Lake, Srebrno jezero Lake (leg. G. Džukić, M. Kalezić 14.04.-15.04.1994, 401, n.spec. = 2); South-eastern Serbia Vlasina Plateau: Promaja (leg. G. Džukić, M. Kalezić, 21/06/2007, 447, n.spec. =1); South-western Serbia Golija Mt.: Dajićko jezero Lake (leg. G. Džukić, 19/09/1969, 442, n.spec. = 1); Jadovnik Mt.: Gvozd (leg. anonim. 27/06/1997, 442, n.spec. =1); Srem Fruška Gora Mt.: Andrevlje (leg. anonim., May 1975, 412, n.spec. =1); (leg. G. Džukić 17/05/1975, 412, n.spec. =1; 25/05/1979, 412); Čortanovci (v.) (leg. M. Mikeš, J. Šoti 05/06/1978, 412, n.spec. = 1); Šuljam (v.), Mutalj spring (leg. G. Džukić 16/04/1980, 412, n.spec. = 2); Inđija: Slankamen (v.) (leg. G. Džukić 10/07/1974, 458, n.spec. = 1); Pećinci: Obrež (v.), Obedska bara pond, Krstonošiča okno (leg. G. Džukić 09/05/1981, 458, n.spec. = 1); Obedska bara pond, Matijevica forest (leg. G. Džukić, M. Kalezić 11/05/2004, 458, n.spec. = 1); Surčin: Dobanovci (v.), Vojvođanska street no. 126 (leg. D. Cirović 15/08/1992, 458, n.spec. = 1); Šid: Morović (v.) - Jamena (v.) (leg. G. Džukić 16/04/1981, 458, n.spec. =2); Šumadija Avala Mt.: Čarapićev Brest (leg. T. Vukov, 08/06/2003, 526, n.spec. = 1); Beograd: Čukarica, Žarkovo (leg. anonim. s. d., 526, n.spec. = 1); Palilula, Lešće cemetery (leg. anonim. 23/05/2000, 444, n.spec. = 1); Rakovica, Monastery Rakovica (leg. G. Džukić 09/05/1969, 444, n.spec. = 1); Straževica hill (leg. anonim. 05/09/1969, 444, n.spec. = 1); Umka, Lipik (leg. M. Milenković 28/06/1981, 444, n.spec. = 1); Zvezdara, Mirijevo (leg. I. Majić April 1972, 526, n.spec. =1); Western Serbia Tara planina Mt.: Bulibanovac (leg. G. Džukić, M. Kalezić, D. Jović, 30/06/2008, 483, n.spec. = 2); Zaovine (v.), Beli Rzav, lake (leg. G. Džukić 20/05/1972, 483, n.spec. = 1); (leg. G. Džukić, M. Kalezić 12.06.-13.06.2001, 
483, n.spec. = 2); Zlatibor Mt.: Gaj (v.), Uvac (r.) - canyon (leg. M. Gvozdić 02/05/1992, 431, n.spec. =1; 31/07/1992, 431); Veliki Rzav (r.) (leg. M. Gvozdić 01/05/1992, 431, n.spec. =1); Zvijezda Mt.: Jagoštica (v.), Veliki Kraj, Čairi (leg. G. Džukić 23/05/1972, 483, n.spec. =1); Slovakia Bratislava(leg. anonim., 24/05/1978, 425, n.spec. $=1$ ); Slovenia Koper Portorož: Dragonja (r.), Dragonja (r.), mouth to Pinjevac (r.) (leg. B. Kryštufek, 01/05/1986, 425, n.spec. =1).

\section{Natrix tessellata}

S. I. (leg. anonim. s. d., 1913, n.spec. $=8$ ); Croatia Baranja Kopački rit: Bilje, Sakadaš Lake (leg. J. Mikuška, 22/06/1970, 393, n.spec. = 1); Zagorje Donja Voća: Ivanac (v.), Vindije cave, bellow (leg. B. Jalžić, 18/07/1976, 393, n.spec. = 1); Macedonia South-western Region Ohridsko jezero Lake: Peštani (v.) (leg. G. Džukić, 27/08/1969, 406, n.spec. =1); Prespansko jezero Lake: Golem Grad (i.) (leg. BIOECO 21/05/2002, 406, n.spec. =3); Vardar Zone Dojransko jezero Lake: Star Dojran - Nov Dojran (leg. G. Džukić, 08/11/1973, 406, n.spec. = 1); Katlanovo: Pčinja (r.) (leg. G. Džukić, S. Petkovski, V. Sidorovska 23/06/1998, 406, n.spec. =1); Montenegro Mediterranean Montenegro Kotor: Radanovići (v.) (leg. A. Hegediš, 01/08/1990, 399, n.spec. $=$ 1); Tivat: Bigovo (v.), crossroad to Bigovo (v.) (leg. A. Hegediš 03/08/1990, 399, n.spec. =1); Tivatske solane (leg. anonim. 29/05/1996, 399, n.spec. = 1); Ulcinj: Ada Bojana, bridge (leg. G. Džukić, M. Kalezić 08/06/2005, 399, n.spec. =1); Northern Montenegro Tara (r.) - canyon: Tepca (v.) (leg. anonim., July 2003, 414, n.spec. = 1; July 2005, 414); (leg. G. Džukić 04/07/1988, 414, n.spec. = 4); Submediterranean Montenegro Podgorica: Morača (r.), Zlatica (leg. K. Ljubisavljević, 03/09/1999, 399, n.spec. =1); Skadarsko jezero Lake: Karuč, beach (leg. V. Kalafatić 17/05/1995, 399, n.spec. =1); Serbia Banat Bela Crkva: Stara Palanka (v.) (leg. anonim., 20/06/1997, 441, n.spec. = 1); (leg. Đ. Novak, L. Novak 15/06/2005, 441, n.spec. = 1); Beograd: Palilula, Krnjača, ponds at the left bank (leg. B. Žiljak 07/04/1996, 441, n.spec. =1); Pančevački rit marsh (leg. M. Anđelković s. d., 441, n.spec. = 1); Deliblatska peščara: Dubovac (v.) (leg. anonim. September 1972, 441, n.spec. =1); Stevanove Ravnice (leg. G. Džukić, M. Kalezić 21/08/1998, 441, n.spec. =1); Kovin: Malo Bavanište (v.), Beli Breg (leg. G. Džukić 25/10/2004, 441, n.spec. = 1); Pančevo: Dunav (r.), downstream from i. Forkontumac, bellow Bele Stene (leg. P. Cakić 31/05/1994, 441, n.spec. =1); surroundings (leg. anonim. 10/08/1996, 441, n.spec. $=1$ ); Central Serbia Ćićevac: surroundings (leg. S. Mićić, 1959, 416, n.spec. =7); Kraljevo: Sirča (v.), Kamidžora (h.) (leg. S. Marinković 07/08/1986, 415, n.spec. = 1); Prokuplje: Toplica (r.), 5-6 km eastern (leg. anonim. 28/08/1961, 415, n.spec. $=1$ ); $15 \mathrm{~km}$ western (leg. anonim. 24/09/1961, 415, n.spec. $=1$ ); Trstenik: Zapadna Morava (r.), left bank (leg. K. Ljubisavljević 23/07/1998, 415, n.spec. =1); Eastern Serbia Bela Palanka: Divljana (v.), Divljansko jezero Lake (leg. Dimić, 21/08/1996, 929, n.spec. =1); Kosovo Novo Brdo hill: Gornji Markeš (v.) (leg. P. Jakšić, 10/04/1986, 929, n.spec. =1); North-eastern Serbia Beljanica Mt. (leg. R. Ajtić, August 1998, 422, n.spec. =1); Donji Milanovac (leg. J. Crnobrnja Isailović, I. Aleksić 29/05/1998, 422, n.spec. =1); Kučajske planine Mts.: Grza (r.), cottage (leg. Đ. Marković 08/07/2009, 422, n.spec. =1); Malinik Mt.: Lazareva Reka (r.) - canyon, exit (leg. G. Džukić 08/07/1982, 422, n.spec. = 2); Zlotska pećina cave (leg. anonim. 15/05/1970, 422, n.spec. =1); Zlot (v.), Lazareva pećina cave - Vernikica, above road (leg. S. Marinković 12/07/1982, 422, n.spec. =1); Zaječar: Trnavac (v.), Majino okno pond (leg. anonim. 23/05/2010, 422, n.spec. =1); North-western Serbia Ljig: Čibutkovica (v.), Vladina bara pond (leg. V. Kalafatić, 12/05/1990, 938, n.spec. = 1); Dudovica (v.) (leg. G. Džukić 17/09/1985, 938, n.spec. =1); Ljubovija: Gornja Trešnjica (v.), Trešnjica (r.) - canyon (leg. G. Džukić 27/05/1972, 938, n.spec. = 2); Trešnjica (r.) canyon, Soko Grad (leg. J. Crnobrnja Isailović 04/04/1998, 938, n.spec. =1); Tribuća (r.) - canyon (leg. anonim. 05/07/1978, 938, n.spec. =1); Maljen Mt.: Divčibare, Rior (leg. anonim. 28/06/2003, 938, n.spec. =1); Šabac: Varna (v.) (leg. S. Petrović 01/10/2005, 938, n.spec. =1); Valjevo: Gradac (r.), spring (leg. S. Stamenković 26/05/1994, 938, n.spec. = 1); Pomoravlje Smederevo: city, city (leg. A. Urošević, 22/04/2011, 471, n.spec. =1); Southern Serbia Medveđa: Maćedonce (v.), Maćedonce (v.), $2 \mathrm{~km}$ toward Sijerina (leg. G. Džukić, 17/05/1977, 929, n.spec. =1); Tulare (v.), Jablanica (r.), $1.5 \mathrm{~km}$ from the village (leg. G. Džukić 16/05/1977, 929, n.spec. =1); Srem Stara Pazova: Belegiš (v.), bellow right enbankment of Dunav (r.) (leg. S. Marinković, 09/04/1989, 471, n.spec. =1); Šumadija Avala Mt.: Trešnja (leg. anonim., 21/06/1990, 948, n.spec. =1); Beograd: 
Ada Ciganlija (i.) (leg. B. Žiljak 10/05/1996, 948, n.spec. = 1); Čukarica, Sremčica, Rakina Bara pond (leg. G. Džukić, M. Kalezić 27/04/1998, 948, n.spec. = 1); Žarkovo, Bele Vode (leg. P. Cakić 29/03/1980, 948, n.spec. = 1); Sava (r.), right bank, 24 km from the mouth (leg. M. Paunović 27/07/1997, 948, n.spec. = 1); Voždovac, Banjica (leg. B. Garzičić 26/05/1979, 948, n.spec. = 1); Vračar, Požarevačka street (leg. B. Žiljak 12/07/1997, 948, n.spec. = 1); Čačak: Ovčar Banja (leg. B. Zorić, D. Zorić 07/07/2005, 948, n.spec. = 1); Gornji Milanovac: Beršići (v.), Dičina (r.) (leg. D. Kataranovski 15.07.-20.07.1993, 948, n.spec. = 1); (leg. anonim. 01/07/2008, 948, n.spec. =1); (leg. D. Kataranovski 15/07/1989, 948, n.spec. = 1); Western Serbia Tara planina Mt.: Derventa $(r$.) canyon, Perućac Lake (leg. anonim., 21/05/1972, 471, n.spec. =1); Užice: Vrutci (v.), Vrutci Lake (leg. M. Marković 12/07/2002, 471, n.spec. = 1); Slovenia Koper Portorož: Dragonja (r.), Dragonja (r.), mouth to Pinjevac (r.) (leg. B. Kryštufek, 01/05/1986, 393, n.spec. =1).

\section{Pituophis catenifer}

USA California Stanislaus County: Modesto area (leg. H. E. Munsterman, 10/05/1954, 1902, $\overline{\text { n.spec. }}=2$ ).

\section{Platyceps najadum}

Bosnia \& Herzegovina Herzegovina Mostar: Blagaj, Svinjski Do (leg. S. Marinković, 02/05/1986, 525, n.spec. =1); Croatia Northern Dalmatia Senj: Jurjevo (v.) (leg. N. Tvrtković, 04/07/1976, 514, n.spec. =1); Zadar: Bokanjac (leg. V. Pavlović 1958, 514, n.spec. =1); Southern Dalmatia Pelješac Peninsula: Mirci, hunting ground (leg. anonim., 26/08/1989, 514, n.spec. $=1$ ); Montenegro Mediterranean Montenegro Boka Kotorska: Luštica Peninsula, Đuraševići beach, Krtole (v.) (leg. V. Stevanović, s. d., 403, n.spec. =1); Budva: Petrovac (leg. anonim. July 1959, 403, n.spec. = 1); (leg. K. Bajić 03/09/1990, 403, n.spec. =1); Submediterranean Montenegro Cetinje: Markovina (leg. G. Džukić, M. Kalezić, M. Marković, 06/06/2005, 400, n.spec. =1); Podgorica: Jelin Dub (leg. G. Džukić, M. Kalezić, M. Marković 03/06/2005, 400, n.spec. = 1); Skadarsko jezero Lake: Murići (v.), 3 km toward Virpazar (leg. anonim. 07/06/1991, 400, n.spec. $=$ 1); Serbia South-eastern Serbia Pčinja (r.) - gorge: Monastery Prohor Pčinjski (leg. J. Crnobrnja, I. Aleksić, 04/06/1998, 525, n.spec. =1).

\section{Telescopus fallax}

Bulgaria Struma (r.) - valley Kresna: "Pejo Javorov" railroad station (leg. M. Langurov, August 2002, 515, n.spec. =1); Croatia Central Dalmatia Brač (i.): Dračevica (v.) - Nerežišća (v.), road (leg. R. Silaški, 10/08/1990, 520, n.spec. =1); Ložišća (v.), Nerežišća (v.) (rad to) (leg. R. Silaški 15/07/1989, 520, n.spec. =1); Split: Kašteli, Opor hill, at Malačka (leg. N. Tvrtković 23/08/1976, 520, n.spec. = 1); Northern Dalmatia Zadar: Bokanjac, Preko (leg. anonim., 1960, 520, n.spec. $=2$ ); (leg. V. Pavlović 1958, 520, n.spec. $=1$ ); Southern Dalmatia Pelješac Peninsula: Mirci, hunting ground (leg. anonim., 16/08/1989, 520, n.spec. $=2$ ); Macedonia Vardar Zone Veles: Vršnik hill, Zlatna Pešter (leg. G. Džukić, 06/11/1973, 515, n.spec. =1); Montenegro Mediterranean Montenegro Kotor: Bigova (v.), Trašte bay (leg. anonim., 21/08/1988, 515, n.spec. $=1)$.

\section{Zamenis longissimus}

S. I. (leg. anonim. s. d., 1915, n.spec. =6); Bosnia \& Herzegovina Bosnia Bugojno: Mračaj (v.), road to the village, above Duboka (leg. anonim., 25/07/1969, 489, n.spec. $=1$ ); Croatia Central Dalmatia Hvar (i.): Brusje (leg. N. Tvrtković, 04/10/1975, 937, n.spec. $=1$ ); Northern Dalmatia Zadar: Bokanjac (leg. M. Radovanović, s. d., 937, n.spec. =1); (leg. V. Pavlović 1958, 937, n.spec. =1); Podravina Varaždin: Varaždin Breg (v.) (leg. N. Tvrtković, June 1976, 937, n.spec. = 1); Posavina Sisak (leg. anonim., spring 1963, 937, n.spec. = 1); Macedonia Southern Mountains Kožuf Mt.: Smrdlivi Vodi (leg. Lj. Janković, 10.05.-18.05.1975, 411, n.spec. = 1); 
Vardar Zone Tikveš: Sopot (leg. J. Mikuška, 09/05/1970, 411, n.spec. = 1); Montenegro Eastern Montenegro Prokletije Mts.: Grbaja valley (leg. J. Crnobrnja, 03/07/1984, 387, n.spec. = 1); Northern Montenegro Tara (r.) - canyon: Tepca (v.) (leg. anonim., 17/07/2003, 387, n.spec. = 1; July 2005, 387); (leg. Lj. Tomović, Đ. Đoković July 2001, 387, n.spec. =1); Submediterranean Montenegro Lovćen Mt.: Ivanova Korita, Bjeloši (v.) - Ivanova Korita (leg. G. Džukić, M. Kalezić, M. Marković, 22/06/2004, 387, n.spec. =1); Podgorica: Mitrovići (v.) (leg. G. Džukić, M. Kalezić, M. Marković s. d., 387, n.spec. = 1); Radovče (v.), Gostilje (leg. G. Džukić, M. Kalezić 09/06/1997, 387, n.spec. =1); Serbia Banat Beograd: Palilula, Krnjača, embankment toward Pančevo (leg. G. Džukić, M. Kalezić, 28/09/2000, 924, n.spec. = 1); Pančevački rit marsh, after the brigde, at the enbankment (leg. M. Anđelković 04/04/2012, 924, n.spec. = 1); Vršački Breg Mt.: Gudurički Vrh (leg. G. Džukić 10/06/1970, 924, n.spec. = 1); Markovac (v.), hunting ground (leg. Đ. Novak June 2004, 924, n.spec. =1); Mesić (v.), $500 \mathrm{~m}$ after crossroad to Jablanka (v.) (leg. G. Džukić 23/09/1992, 950, n.spec. = 1); Mesićki potok (leg. Đ. Novak 15.06.-30.06.2004, 949, n.spec. $=2 ; 950 ; 28.06 ., 30.06 .2004$, 949; June 2004, 949); (leg. G. Džukić 12/05/2004, 950, n.spec. =1); Mesićki potok, bridge at old road to Kuštilj (v.) (from Vršac), near Tri bunara (leg. Đ. Novak 01/06/2005, 950, n.spec. =1); Široko Bilo (leg. G. Džukić 12/06/1970, 924, n.spec. =1); Central Serbia Ćićevac: surroundings (leg. S. Mićić, 1959, 927, n.spec. =1); Jastrebac Mt.: Ravnište, Stogovačka Kosa (leg. S. Marinković 18/10/1988, 397, n.spec. =1); Kraljevo: Savovo (v.) (leg. anonim. 13/07/1977, 927, n.spec. =1); Kruševac: Kukljin (v.), $1.5 \mathrm{~km}$ before Kukljin (v.) from Jasika (v.) (leg. G. Džukić, M. Kalezić 06/05/2001, 397, n.spec. =1); Kuršumlija (leg. anonim. November 1949, 927, n.spec. =1); Trstenik: Donja Crnišava (v.), near the river (leg. K. Ljubisavljević 27/07/1997, 397, n.spec. = 1); Stena hill (leg. J. Marković 17/07/1998, 397, n.spec. = 2); Zbeg hill (leg. T. Luković 02/05/1998, 397, n.spec. =1); Eastern Serbia Pirot: Šugrin (v.), Kalna (leg. J. Crnobrnja, I. Aleksić, 01/06/1998, 366, n.spec. =1); Stara planina Mt.: Topli Do (v.), 4 km above the village (leg. Z. Franolić 27/07/1983, 366, n.spec. = 1); Svljig: 2 $\mathrm{km}$ toward Knjaževac, $2 \mathrm{~km}$ toward Knjaževac (leg. D. Jović 22/06/2008, 366, n.spec. =1); Kosovo Šar planina Mts.: Drajkovce (v.), Lepenac (r.) (leg. P. Jakšić, 05/06/1987, 421, n.spec. = 1); Žar planina Mt., Mušutište (v.), Rusenica gorge, Monastery Sveta Trojica (leg. G. Džukić 05/07/1979, 421, n.spec. =1); Metohia Prizren: Vrbnica (v.) (leg. G. Džukić, 07/07/1969, 421, n.spec. =1); Prokletije Mts.: Junička planina Mt., Đocaj (v.), above (leg. G. Džukić 04/06/1977, 421, n.spec. = 1); North-eastern Serbia Beljanica Mt. (leg. R. Ajtić, 07/07/1998, 419, n.spec. = 1; July 2006, 419); Despotovac: Resavica (v.), $2 \mathrm{~km}$ toward Ravna Reka (v.) (leg. G. Džukić, M. Kalezić 12/05/2002, 419 , n.spec. =1); toward Vražja bara pond (leg. G. Džukić, M. Kalezić 12/05/2002, 419, n.spec. =1); Donji Milanovac: Lepenski Vir (leg. J. Crnobrnja Isailović, I. Aleksić 29/05/1998, 396, n.spec. =1); Golubac: Dobra (v.), Dobra (v.) - Gornji Milanovac (leg. G. Džukić, M. Kalezić 14/07/1998, 396, n.spec. =1); toward Donji Milanovac (leg. M. Milenković 10/07/1989, 396, n.spec. =1); Kladovo: Ram (v.), Gorica, footill (leg. G. Džukić, M. Kalezić 17/09/2003, 396, n.spec. =1); Kučevo (leg. J. Mikuška June 1967, 396, n.spec. =1); Malinik Mt.: Zlot (v.), Lazareva pećina (leg. G. Džukić 18/09/1982, 419, n.spec. = 1); Lazareva pećina - Vernikica, bellow road (leg. G. Džukić 12/07/1982, 419, n.spec. =1); Lazareva pećina cave - Vernikica, above road (leg. S. Marinković 09/07/1982, 419, n.spec. =1); North-western Serbia Bogatić: Zasavica pond, Paljevine (leg. M. Stanković, 22/06/1996, 457, n.spec. = 2); Ljig: Poljanice (v.) (leg. D. Kataranovski 18/06/2005, 457, n.spec. =1); Slavkovica (v.), $1 \mathrm{~km}$ toward Rajac (leg. anonim. 15/06/1991, 457, n.spec. = 1); Ljubovija: Gornje Košlje (v.), Zmijaruge (leg. S. Marinković 26/05/1986, 457, n.spec. = 1); Pomoravlje Paraćin: Izvor (v.), Grza (r.) (leg. S. Eurović, $12 / 07 / 2010,462$, n.spec. $=1$ ); Veliko Gradište: Zatonje (v.), entrance to Zatonje (v.) from Ram (v.) (leg. G. Džukić 27/05/2011, 462, n.spec. =1); South-eastern Serbia Kozjak Mt.: Monastery Prohor Pčinjski (leg. R. Ajtić, 2002, 423, n.spec. =1); Starac Mt. (leg. G. Džukić 19/07/2004, 423, n.spec. = 1); Vlasina Plateau (leg. G. Džukić, M. Kalezić 22/04/2004, 423, n.spec. $=1$ ); Southern Serbia Vranje: surroundings (leg. D. Nedeljković, July-August 1958, 423, n.spec. $=2$ ); Srem Fruška Gora Mt.: Andrevlje (leg. G. Džukić, 25/05/1979, 426, n.spec. $=1$ ); Čortanovci (v.) (leg. M. Mikeš, J. Šoti 30/05/1978, 410, n.spec. = 5; 426; 05/06/1978, 410); (leg. M. Pantović 19/05/1984, 426, n.spec. = 1); Iriški Venac, Brankovac (leg. D. Bejaković 19/10/1987, 410, n.spec. = 1); Osovlje (leg. G. Džukić 25/05/1979, 426, n.spec. = 3); Sremska Kamenica, old road to 
Iriški Venac (leg. D. Mitić 20/05/1978, 439, n.spec. = 1); Sremski Karlovci, near Čarda (leg. $L j$. Tomović 29/05/2002, 439, n.spec. = 1); Zmajevac (leg. Z. Dunđerski 22/05/1975, 439, n.spec. =1); (leg. anonim. 1956, 439, n.spec. = 1); Novi Sad: Petrovaradin, Petrovaradinski rit marsh, Tekija Majur, near Church Marija Snežna (leg. J. Purger 21/05/1991, 439, n.spec. =1); Pećinci: Obrež (v.), Obedska bara pond, hotel (leg. S. Đorđević 15/09/2012, 439, n.spec. = 1); Šumadija Avala Mt.: Čarapićev Brest, Ladan izvor (leg. S. Stamenković, 04/07/1994, 517, n.spec. = 1); (leg. D. Pavićević 18/10/1985, 517, n.spec. =1); (leg. G. Džukić 01/06/2002, 517, n.spec. =2); Trešnja, Rasadnik, Lipar, 5 km before Trešnja (leg. P. Đorđević 20/05/1987, 517, n.spec. =1); (leg. anonim. 19/10/1998, 517, n.spec. = 1); Beograd: Barajevo, Vranić (v.) (leg. S. Đorđević 07/07/1996, 524, n.spec. =1); Grocka, Leštane (v.), Bubanj Potok (leg. V. Cvetković 21/06/2003, 524, n.spec. =1); Palilula, Dimitrije Tucović street (leg. anonim. 08/08/1987, 524, n.spec. =1); Karaburma, Višnjička street (leg. V. Stevanović 08/10/1985, 524, n.spec. = 1); Umka, Karaula hill (leg. M. Milenković 01/05/1986, 524, n.spec. = 1); Lipik (leg. M. Milenković spring 2008, 524, n.spec. = 1); Lipik, Karaula hill (leg. M. Milenković 22/06/1991, 524, n.spec. = 1); Gornji Milanovac: Beršići (v.), Dičina (r.) (leg. D. Kataranovski 15.07.-20.07.1993, 478, n.spec. $=1$ ); Smederevo: Orešac, $1 \mathrm{~km}$ toward Belgrade (leg. M. Paunović 30/04/2003, 478, n.spec. =1); Western Serbia Bajina Bašta: Drina (r.), Perućac Lake (leg. G. Džukić, 14/06/1969, 409, n.spec. = 1;23/06/1973, 409).

\title{
Zamenis situla
}

S. I. (leg. anonim. s. d., 1916, n.spec. = 2); Croatia Central Dalmatia Biokovo Mt.: Kozice (v.), road to Šošići (v.) (leg. N. Tvrtković, 28/08/1976, 435, n.spec. =1); Northern Dalmatia Knin: surroundings (leg. M. Stajić, begining of August 1995, 435, n.spec. =1); Southern Dalmatia Pelješac Peninsula: Lovište (v.) (leg. D. Kataranovski, 10/06/1987, 435, n.spec. = 1); Macedonia Vardar Zone Katlanovo: Divle (v.), Raklje (leg. G. Džukić, M. Belanović, spring-summer 1978, 433, n.spec. = 1); Negotino: Krivolak (leg. A. Ćetković 25/03/1989, 433, n.spec. =1).

\section{ELAPIDAE}

\section{Micrurus sp.}

Venezuela Piara Fe San Isidoro (leg. Š. Talić, 1962, 1908, n.spec. =1).

\section{ERYCIDAE}

\section{Eryx jaculus}

Macedonia Vardar Zone Kočani: Orizari (v.) (leg. S. Petkovski, s. d., 373, n.spec. =1).

\section{LAMPROPHIIDAE}

\section{Atractaspis sp.}

Liberia Monrovia Suakoko (leg. anonim., s. d., 1907, n.spec. =1).

\section{Boaedon fuliginosus}

Liberia Monrovia Suakoko (leg. anonim., s. d., 1905, n.spec. =1).

\author{
Lycophidion sp. \\ Liberia Monrovia Suakoko (leg. anonim., s. d., 1906, n.spec. =1).
}




\title{
Malpolon insignitus
}

S. I. (leg. anonim. s. d., 1914, n.spec. =1); Bosnia \& Herzegovina Herzegovina Čapljina: Domanovići (v.) (leg. S. Marinković, 13/07/1986, 380, n.spec. =1); Jetrenica: peak (leg. S. Marinković 09/07/1988, 380, n.spec. = 1); Croatia Northern Dalmatia Pag (i.): Malo Blato (leg. N. Tvrtković, 21/07/1977, 450, n.spec. = 1); Zadar: Bokanjac (leg. M. Radovanović June 1960, 450, n.spec. = 1); (leg. N. Tvrtković end of June 1976, 450, n.spec. = 1); Southern Dalmatia Dubrovnik Trebinje: Ukoplje (v.) (leg. G. Džukić, 24/06/1984, 450, n.spec. =1); Pelješac Peninsula: Janjina (leg. P. Jakšić 16/05/1987, 450, n.spec. = 1); Libya Cyrinaica Magrun (leg. A. Ćetković, 04/06/1988, 388, n.spec. = 1); Macedonia Vardar Zone Gradsko: surroundings (leg. G. Džukić, 15/04/1972, 424, n.spec. = 1); Pčinja (r.) - gorge: exit toward Veles (leg. G. Džukić 24/06/1998, 424, n.spec. =1); Montenegro Mediterranean Montenegro Ulcinj: Ada Bojana (leg. A. Gliksman, 05/09/1995, 459, n.spec. = 1); Donji Štoj (v.), crossroad to Ada Bojana (leg. anonim. 23/06/2004, 459, n.spec. =1); Submediterranean Montenegro Skadarsko jezero Lake: Beška (i.) (leg. $B$. Kryštufek, 07/06/1990, 459, n.spec. =1); Rijeka Crnojevića - Virpazar, crossroad to Dodoši (v.) (leg. anonim. 22/06/2004, 459, n.spec. =1); Poseljani (v.), near the village (leg. anonim. 22/06/2004, 459 , n.spec. $=1)$; Starčevo (i.) (leg. anonim. 06/06/1990, 459, n.spec. $=1)$.

\section{TYPHLOPIDAE}

\section{Xerotyphlops vermicularis}

Macedonia South-western Region Prespansko jezero Lake: Pretor (v.) (leg. G. Mesaroš, s. d., 413, n.spec. = 2); Vardar Zone Demir Kapija: Udovo (v.) (leg. BIOECO, 10/06/2006, 413, n.spec. = 3); Pčinja (r.) (leg. anonim. 11/05/1952, 474, n.spec. = 4); Stari Dojran (leg. anonim. 22/05/1958, 379, n.spec. $=10 ; 14 / 05 / 1966,379)$; Montenegro Mediterranean Montenegro Kotor: Bigova (v.), Trašte bay (leg. anonim., 21/08/1988, 413, n.spec. =1).

\section{VIPERIDAE}

\section{Atheris squamigera}

Kenya Victoria Lake Kakamego forest (leg. G. Nonveiller, 1986, 1901, n.spec. =1).

\author{
Bitis gabonica \\ Liberia Monrovia Suakoko (leg. anonim., s. d., 1903, n.spec. =1).
}

\section{Vipera ammodytes}

S. I. (leg. anonim. s. d., 1918, n.spec. = 16; 1919); Bosnia \& Herzegovina Bosnia Bosansko Grahovo: bend to Knin (leg. anonim., s. d., 932, n.spec. =1); Śipovo: Babići (v.), Sokočnica (r.) - canyon (leg. Lj. Tomović, R. Ajtić 08/08/2001, 932, n.spec. =1); Vaganac (r.) - canyon (leg. Lj. Tomović, R. Ajtić 03.08.-12.08.2001, 932, n.spec. = 4); Herzegovina Livno - Glamoč: Carevica (v.) (leg. G. Džukić, 18/05/1983, 472, n.spec. =1); Mostar: Blagaj, Herceg-Stjepanov grad (leg. anonim. s. d., 472, n.spec. =1); Popovo Polje: Bjelasnica Mt., Mrkonjić, Kumbara peak (leg. $S$. Marinković 26/04/1986, 472, n.spec. =1); Stolac: Fatnica (v.) (leg. G. Džukić 14/05/1983, 496, n.spec. =1); Trebinje (leg. anonim. s. d., 496, n.spec. =3); Bulgaria Istočni Rodopi Mt. Bela Reka (leg. Lj. Tomović, July 1998, 392, n.spec. =1); Croatia Central Dalmatia Biokovo Mt.: Vošac, "Vošac", mountain house (leg. G. Džukić, 25/07/1969, 465, n.spec. =1); Lika Velebit Mt.: Mirovo (leg. N. Tvrtković, B. Kryštufek, 15/08/1976, 465, n.spec. = 1); Oštarije - Brušan (leg. anonim. 12/04/1989, 465, n.spec. =1); Macedonia Eastern Mountains Kratovo: Stracin (v.), plateau (leg. BIOECO, 19/05/2002, 487, n.spec. =1); Southern Mountains Kozjak Mt.: Alšar, Rožden (leg. anonim., s. d., 487, n.spec. =1); South-western Region Galičica Mt. (leg. anonim., s. d., 485, 
n.spec. = 1); Prespansko jezero Lake: Golem Grad (i.) (leg. BIOECO 21/05/2002, 485, n.spec. = 5); Oteševo (v.) (leg. BIOECO 23/05/2002, 485, n.spec. =1); Vardar Zone Bogdanci: Pogana hill (leg. D. Pavićević, 04/05/1985, 487, n.spec. $=1$ ); Montenegro Central Montenegro Kolašin: Morača (r.) (leg. anonim., s. d., 455, n.spec. =4); (leg. anonim. s. d., 455, n.spec. =3); Mrtvica (r.) canyon (leg. Lj. Tomović, R. Ajtić 27/07/2001, 455, n.spec. =1); Eastern Montenegro Prokletije Mts.: Đebeza Mt. (leg. G. Džukić, M. Kalezić, M. Marković, 03/05/2005, 407, n.spec. =1); Grbaja valley, bellow Vojuša (leg. R. Mandić 30/06/1984, 407, n.spec. =1); Mateševo, $9 \mathrm{~km}$ from Mateševo toward Veruša (leg. anonim. 10/06/1991, 407, n.spec. $=1$ ); Mediterranean Montenegro Boka Kotorska: Risan, sharp curves, before crosssroad Crkvice - Ledenice (leg. G. Džukić, 04/06/1984, 378, n.spec. = 1); Budva: Petrovac (leg. S. Simić 10/07/1977, 378, n.spec. = 1); Tivat: Lastva Grbaljska (leg. anonim. 27/05/1996, 378, n.spec. =1); Ulcinj: Ada Bojana (leg. G. Pasuljević 23/06/1976, 378, n.spec. = 1); (leg. J. Radojičić 02/05/1998, 378, n.spec. = 1); (leg. T. Perezić June 1975, 378 , n.spec. $=1$ ); Northern Montenegro Durmitor Mt.: Boljske Grede, foothill (leg. anonim., s. d., 480, n.spec. $=1$ ); Grabovica (v.) (leg. anonim. s. d., 480, n.spec. $=1$ ); Komarnica (r.), Nevidio canyon, bellow Žute Grede (leg. G. Džukić 22/08/1984, 480, n.spec. = 1); Pirlitor (leg. anonim. 05/08/1990, 480, n.spec. = 1); Šavnik: Duži (v.), Žglijeb (leg. G. Džukić 08/07/1971, 480, n.spec. =1); Kutnja Njiva, Džukića tower (leg. G. Džukić 08/07/1971, 480, n.spec. =1); Petnjica (v.), Džukov Do, above (leg. G. Džukić 24/07/1984, 480, n.spec. =1); Tara (r.) - canyon: Bajlovića Sige (leg. anonim. s. d., 484, n.spec. =6); (leg. D. Bejaković 21.08.-28.08.1987, 484, n.spec. = 1; August 1988, 484); Lever Tara (leg. anonim. s. d., 936, n.spec. $=1$ ); Tepca (v.) (leg. Lj. Tomović, R. Ajtić different years, 921, n.spec. = 10; 931; July 2002, 428); (leg. R. Ajtić 24/07/2003, 936, n.spec. = 3); (leg. anonim. s. d., 936, n.spec. =2); Submediterranean Montenegro Lovćen Mt.: Međuvršje (leg. G. Džukić, 02/06/1984, 470, n.spec. = 1); Orjen Mt.: Siljevica (leg. M. Milenković 31.05.-03.06.1979, 470, n.spec. = 3); Vrbanje (v.), Pod Duge (h.) (leg. M. Belanović 19.05.-23.05.1984, 470, n.spec. = 3); (leg. G. Džukić 15/06/1972, 470, n.spec. $=1$ ); Podgorica: Piperi, $10 \mathrm{~km}$ south from Podgorica (leg. anonim. 12/07/2002, 479, n.spec. = 1); Spuž (leg. anonim. 20/09/1995, 479, n.spec. = 2; s. d., 479); Skadarsko jezero Lake: Bračeni (v.), Virpazar - Rijeka Crnojevića, road (leg. V. Kalafatić 16/09/1992, 382, n.spec. $=1$ ); Mala Čakovica (i.) (leg. anonim. s. d., 382, n.spec. =1); Virpazar, Besac, toward Godinje (v.) (leg. G. Džukić, M. Kalezić, M. Marković 31/05/2003, 382, n.spec. =1); Vranjina Peninsula (leg. anonim. 09/07/1969, 382, n.spec. = 2); (leg. Lj. Tomović, R. Ajtić 05/05/2009, 382, n.spec. $=$ 2); Western Montenegro Nikšić: Velimlje (v.), Tupan (v.) (leg. anonim., 10/08/1976, 473, n.spec. = 1); Vilusi (v.), Krivača (leg. M. Bulajić September 1985, 473, n.spec. =1); Orjen Mt.: Grahovo, dam (leg. anonim. s. d., 473, n.spec. = 1); Kovačevići (leg. anonim. s. d., 473, n.spec. =1); Serbia Banat Bela Crkva: Kusić (v.), Nera (r.) (leg. anonim., 23/10/2005, 513, n.spec. =1); Central Serbia Ćićevac: Stalać, Stevanac (leg. R. Ajtić, 23/03/2005, 493, n.spec. =2); Goč Mt.: "Dobre Vode", mountin house (leg. K. Vasić 23/05/1977, 942, n.spec. = 1); Jastrebac Mt.: Buci (v.) (leg. D. Kataranovski 09/09/1979, 942, n.spec. =1); Kopaonik Mt.: Brzeće (v.) (leg. Lj. Tomović, R. Ajtić 27.08.-28.08.2000, 372, n.spec. = 3); Jošanička Banja, Gornje and Donje Čamage (leg. anonim. 16/07/1987, 372, n.spec. = 1); Šanac (leg. Lj. Janković 24/08/1973, 372, n.spec. = 1; May 1968, 372); (leg. A. Vlajić 02/05/1986, 372, n.spec. = 1); Kruševac: Ćelijsko jezero Lake (leg. R. Ajtić June 2004, 942 , n.spec. =1); Kuršumlija: Vlahinja (v.) (leg. B. Milenković 02/07/1979, 942, n.spec. =2); (leg. S. Obradović 10/09/1978, 942, n.spec. = 1); Prokuplje: Donja Konjuša (v.) (leg. D. Gagulić 13/09/1975, 362, n.spec. =1); Viča (v.), Ložđe hill (leg. Lj. Tomović 15.06.-17.07.1999, 362, n.spec. = 5); Vodice (v.) (leg. M. Petković 15/05/1981, 362, n.spec. = 1); Trstenik: Donja Crnišava (v.), Zbeg hill (leg. J. Luković 24/07/1997, 493, n.spec. = 1); (leg. anonim. s. d., 493, n.spec. = 2); Eastern Serbia Dimitrovgrad: Smilovci (v.), surroundings (leg. anonim., 15/07/1957, 359, n.spec. $=3$ ); (leg. anonim. s. d., 359, n.spec. =1); Rtanj Mt.: Baba (leg. Z. Dunđerski 03/05/1977, 475, n.spec. =1); (leg. anonim. May 1998, 475, n.spec. $=1$; s. d., 475); Sićevačka klisura gorge: Oblik (leg. Z. Romčević 05/07/1997, 529, n.spec. =1); Roman road (leg. R. Ajtić 24/06/2005, 529, n.spec. = 1); Soko Banja: Bovan (v.), Bovansko jezero Lake (leg. anonim. 30/06/1998, 529, n.spec. $=1$ ); Stol Mt.: plateau, 500-600 m from the mountain house (leg. D. Pavićević 19/05/1989, 475, n.spec. =1); Suva planina Mt.: Lužnička Reka (r.) - gorge (leg. anonim. 09/05/2000, 529, n.spec. =1); Kosovo Gnjilane: Buzalak (v.), Dobri Čiča hill (leg. G. Džukić, 17/05/1977, 361, n.spec. = 1); Končulj (v.) (leg. anonim. begining of August 2004, 361, n.spec. =1); Slivovo (v.) (leg. J. Simić 07/09/1978, 361, n.spec. = 2); Grmija hill (leg. G. Pasuljević 20/04/1970, 361, n.spec. = 1); Ibar (r.) - valley: Ibarska Slatina, Ceranjska Reka (r.) (leg. G. Pasuljević 08/07/1975, 386, n.spec. = 1); (leg. G. Pasuljević 29/06/1975, 386 , n.spec. $=1 ; 07 / 08 / 1975,386 ; 15 / 08 / 1975,386$ ); Leposavić: Gnježdane (v.) (leg. G. Pasuljević 
24/05/1971, 503, n.spec. = 1); Kijevčiće (v.) (leg. G. Pasuljević, R. Kostović 06/05/1973, 503, n.spec. = 2); Koporiće (v.) (leg. G. Pasuljević 08/06/1971, 503, n.spec. = 1; 10/06/1971, 503); Kruševo (v.) (leg. R. Kostović, G. Pasuljević 06/05/1973, 497, n.spec. = 1); Sočanica (v.) (leg. R. Novaković 12/06/1978, 497, n.spec. = 2; 08/07/1978, 497); Zavrata (v.) (leg. G. Pasuljević 18/06/1971, 503, n.spec. = 1); (leg. G. Pasuljević, R. Kostović 01.05.-15.05.1973, 497, n.spec. = 2); Novo Brdo hill: above flotation (leg. G. Pasuljević 24/07/1970, 361, n.spec. =1); Bostane (v.) (leg. P. Jakšić s. d., 361, n.spec. =1); Jasenovik (v.) (leg. P. Jakšić 25/05/1986, 361, n.spec. =1); Trnićevce (v.) (leg. $P$. Jakšić May 1985, 361, n.spec. = 1); (leg. P. Jakšić 29/05/1985, 361, n.spec. = 1); Šar planina Mts.: Brezovica, Prljevački Potok (leg. P. Jakšić 17/07/1986, 477, n.spec. = 1); Prevalac (leg. anonim. 28/08/1979, 477, n.spec. = 1); Uroševac: Gornje Nerodimlje (v.) (leg. anonim. 10/07/1976, 477, n.spec. = 1; 20/07/1976, 477; 20/08/1976, 477); (leg. anonim. 15/03/1977, 477, n.spec. = 1); Vučitrn: Gornja Sudimlja (v.) (leg. R. Grguri 09/06/1977, 386, n.spec. =1); Zubin Potok: Lučka Reka (v.) (leg. S. Bašanović 30/09/1976, 386, n.spec. =1); Zvečan: Banjska (v.) (leg. D. Todorović 28/09/1978, 386, n.spec. = 1); Grižani (v.) (leg. D. Todorović 26/05/1979, 386, n.spec. =1); (leg. G. Pasuljević s. d., 488, n.spec. = 13); Metohia Dečani: Dečanska Bistrica (r.) - gorge (leg. V. Stevanović, 12/07/1977, 528, n.spec. =1); Đakovica: Babaj Boks (v.), Babaj Boks (v.) - Deva (v.) (leg. G. Džukić 18/07/1980, 528, n.spec. = 1); (leg. G. Pasuljević, G. Džukić 18/07/1980, 528, n.spec. = 1); Grebnička planina Mt.: Miruša (r.) (leg. B. Milošević, G. Pasuljević 29/06/1980, 492, n.spec. = 1); Miruša (r.) canyon (leg. Devtić, Todorović 20/05/1977, 492, n.spec. = 1); Istok: Mojstir (v.) (leg. D. Lakušić 26/09/1978, 491, n.spec. = 1); Osojane (v.) (leg. D. Lakušić 24/04/1977, 491, n.spec. = 1); Šaljinovica (v.) (leg. D. Lakušić 24/05/1978, 491, n.spec. = 1); Klina: Iglarevo (v.) - Kijevo (v.) (leg. G. Pasuljević 12/09/1975, 492, n.spec. = 1); Volujak (v.) (leg. B. Milošević, G. Pasuljević 01/07/1980, 492, n.spec. =1); Koritnik Mt.: Rapča (v.) (leg. M. Hajredini 31/03/1980, 491, n.spec. =1); (leg. M. Hamza 02/05/1981, 491, n.spec. = 1); Orahovac (leg. S. Trajković 25/08/1976, 492, n.spec. = 1); Prizren: Lokvica (v.) (leg. anonim. 07/09/1978, 528, n.spec. = 1); Sredska (v.) (leg. I. llić 12/08/1976, 528, n.spec. = 1); (leg. I. Nikolić 1976, 528, n.spec. = 1); Vrbičane (v.) (leg. M. Petrović 01/08/1979, 528, n.spec. =1); (leg. M. Hamza 10/05/1979, 528, n.spec. = 1); Prokletije Mts.: Lazović (leg. I. Krizmanić 11/06/1997, 492, n.spec. = 1); Suva Reka: Dulje (v.) (leg. anonim. 10/09/1977, 491, n.spec. = 1); North-eastern Serbia Beljanica Mt.: Sokolica, foothill (leg. anonim., s. d., 481, n.spec. = 2); mountain house (leg. R. Ajtić 01/07/1998, 481, n.spec. =1); Kučajske planine Mts.: Mikuljska Reka (r.) - canyon (leg. anonim. 02/06/1983, 481, n.spec. = 2); (leg. R. Ajtić July 2006, 481, n.spec. = 1); Kučevo (leg. anonim. April 1963, 482, n.spec. = 1); Malinik Mt.: Lazareva Reka (r.) - canyon, near Prerasti (leg. G. Džukić 09/07/1982, 481, n.spec. =1); (leg. I. Krizmanić 20/05/1994, 481, n.spec. = 1; 21/05/1994, 481); Svilajnac (leg. anonim. 17/10/1995, 482, n.spec. =2; s. d., 482); North-western

Serbia Loznica: Paskovac (v.) (leg. anonim., s. d., 467, n.spec. $=10 ; 476 ; 490 ; 494 ; 495$ ); Zajača (v.), Krivi Potok (leg. M. Popović - Fant 01/02/1990, 476, n.spec. = 1); (leg. M. Popović - Fant 05/04/1990, 476, n.spec. = 1); Ljubovija: Gornja Trešnjica (v.), Trešnjica (r.) - canyon (leg. G. Džukić 27/05/1972, 464, n.spec. = 1); Trešnjica (r.) - canyon (leg. anonim. 05/07/1978, 464, n.spec. = 3; s. d., 464); Mali Zvornik: Trešnjica (r.) - canyon, Jerinin Grad (leg. anonim. s. d., 464, n.spec. = 1); Košlje (v.) (leg. anonim. s. d., 464, n.spec. =1); Maljen Mt.: Brežđe (v.) (leg. M. Anđelković, A. Urošević 08/06/2017, 360, n.spec. = 1); Divčibare, Divčibare (leg. D. Kataranovski, V. Cvetković 17/05/1994, 360, n.spec. = 1); Paljba (leg. anonim. June-July 1953, 360, n.spec. = 1); Rior (leg. anonim. 28/06/2003, 360, n.spec. =1); Medvednik Mt. (leg. M. Radovanović 22/07/1949, 360, n.spec. = 1); Mionica: Paštrić (v.), near Ribnička pećina cave (leg. M. Đurić 14/05/2005, 940, n.spec. = 1); (leg. G. Džukić 28/05/1980, 940, n.spec. = 1); Povlen Mt.: Debelo Brdo hill, Stubice hill (leg. R. Ajtić 29/06/1999, 940, n.spec. = 3); Srednji Povlen Mt., Kneževo Polje (leg. D. Jovanović 17/06/2001, 940, n.spec. = 1); (leg. D. Jovanović s. d., 940, n.spec. = 1); Suvobor Mt.: Pranjani (v.) (leg. $D$. Kataranovski, V. Cvetković 17/05/1994, 464, n.spec. =1); Valjevo: Donje Leskovice (v.) (leg. anonim. 14/09/2009, 438, n.spec. =10; s. d., 436; 437); Gradac (r.), Suvaja, between Bogatić (v.) and Lelić (v.), $3 \mathrm{~km}$ downstream from Lelić (v.) (leg. A. Hegediš 28/05/1992, 449, n.spec. = 1); (leg. anonim. 24/06/2004, 449, n.spec. = 1); (leg. I. Krizmanić 25/05/1995, 449, n.spec. = 1); Jablanica (r.) (leg. $I$. Krizmanić 31/07/1991, 449, n.spec. = 1); Petnica (v.), Bujačić (leg. S. Stamenković 22/05/1985, 449, n.spec. = 1); Pomoravlje Golubac (leg. anonim., 29/04/1986, 368, n.spec. = 1); Jagodina: Dragoševac (v.) (leg. G. Džukić, M. Kalezić 17/06/2001, 368, n.spec. =1); South-eastern Serbia Starac Mt.: Pčinja (r.) - valley (leg. Lj. Tomović, R. Ajtić, 24/05/2009, 935, n.spec. = 2); (leg. R. Ajtić July 2007, 935, n.spec. = 3); Starac (v.), Donji Starac (leg. R. Ajtić July 2004, 935, n.spec. = 1); Gornji Starac (leg. Lj. Tomović, R. Ajtić 05/05/2002, 390, n.spec. = 3); Gornji Starac (v.), Čivčije (h.) (leg. Lj. Tomović, R. Ajtić 06/05/2002, 390, n.spec. = 2); Trgovište: Crnovska Reka (r.) - gorge (leg. Lj. 
Tomović, R. Ajtić 29/04/2000, 390, n.spec. =1); Southern Serbia Lebane: Grgurevci (v.), Šumanska Reka (r.) - gorge (leg. anonim., 30/04/2002, 930, n.spec. =1); Medveđa: Maćedonce (v.) (leg. Lj. Tomović 30/04/2002, 930, n.spec. =1); Vranje: Kriva Feja (v.) (leg. anonim. s. d., 930, n.spec. =1); South-western Serbia Giljeva Mt.: Raždaginja (v.) (leg. G. Pasuljević, H. Mesić, 1974, 391, n.spec. = 1); Ibarski Kolašin (leg. I. Ristović 28/04/1977, 391, n.spec. =1); Novi Pazar: Vranovina (v.) (leg. anonim. 05/05/1977, 391, n.spec. =1); Ozren Mt.: Dubočica (r.) - gorge (leg. R. Aitić s. d., 934, n.spec. = 5); Pešter Plateau: Sjenica, Krš (leg. D. Mijajlović 15/08/1976, 391, n.spec. =1); Prijepolje: Divci (v.) (leg. anonim. 12/07/1996, 933, n.spec. = 2); Karoševina (v.) (leg. anonim. 10/06/1996, 933, n.spec. = 2); Ninčići (v.) (leg. anonim. 15/06/1996, 933, n.spec. =1); Seljašnica (v.) (leg. anonim. 15/06/1996, 933, n.spec. = 3); (leg. anonim. 29/09/2005, 404, n.spec. = 7; s. d., 398); Šumadija Bukulja Mt. (leg. anonim., s. d., 486, n.spec. = 3); Gornji Milanovac: Vraćevšnica (v.) (leg. anonim. s. d., 504, n.spec. $=5 ; 505 ; 507$ ); Western Serbia Bajina Bašta: Drina (r.), Perućac Lake (leg. G. Džukić, 21/05/1972, 468, n.spec. =1); Mokra Gora Mt.: Šargan (leg. anonim. July 1958, 468, n.spec. = 1); Mučanj Mt.: Anatema (leg. A. Rohalj 17/08/1989, 466, n.spec. =1); Glog (leg. A. Rohalj 17/08/1989, 466, n.spec. = 1); Požega: Bakionica (v.), Zdravčići (leg. anonim. s. d., 466, n.spec. $=1$ ); Tara planina Mt.: Derventa $(\mathrm{r}$.) - canyon, $2 \mathrm{~km}$ from the mouth (leg. anonim. s. d., 468, n.spec. = 1); Zlatibor Mt.: Gaj (v.), Uvac (r.) - canyon (leg. M. Gvozdić 31/07/1992, 466, n.spec. $=1$ ); Ljubiš (v.) (leg. I. Krizmanić 21/07/2000, 466, n.spec. =1); railroad station "Zlatibor" (leg. S. Stamenković 07/08/2004, 466, n.spec. =1); Zvijezda Mt.: Jagorštica (v.), Galine (leg. G. Džukić 21/05/1972, 468, n.spec. = 1); Veliki Kraj, Vidača (leg. G. Džukić 23/05/1972, 468, n.spec. = 1); (leg. I. Krizmanić 16/06/1994, 468, n.spec. =1); Slovenia Kranj Sveti Jošt (leg. B. Kryštufek, summer 1972, 367, n.spec. $=1)$.

\section{Vipera berus}

S. I. (leg. anonim. s. d., 1917, n.spec. =1); Bosnia \& Herzegovina Herzegovina Kalinovik Mt. (leg. anonim., 1912, 370, n.spec. =1); Zelengora Mt.: Gornje Bare (leg. G. Džukić 13/05/1983, 370, n.spec. =1); Croatia Slavonija Ilok: surroundings (leg. anonim., April 1989, 370, n.spec. =1); Macedonia South-western Region Pelister Mt.: Široka (leg. G. Džukić, 22/07/1979, 499, n.spec. = 3); Montenegro Eastern Montenegro Bjelasica Mt.: Lalevića Dolovi (leg. Lj. Tomović, R. Ajtić, 22/07/2000, 371, n.spec. = 3; 22/07/2003, 371); (leg. Lj. Tomović, $R$. Ajtić, J. Crnobrnja Isailović 23/07/2002, 369, n.spec. =6); Northern Montenegro Durmitor Mt.: Barno jezero Lake (leg. G. Džukić, s. d., 498, n.spec. =1); Barno jezero Lake - Zminje jezero Lake (leg. S. Stoiljković 10/07/1983, 498, n.spec. = 1); Crepulj Poljana, road to Donja Ališnica (leg. A. Ćetković 19/08/1986, 498, n.spec. =1); Malo Škrčko jezero Lake (leg. anonim. 23/07/2004, 498, n.spec. = 1); Pištaline (leg. A. Ćetković 09/09/1987, 498, n.spec. = 1); Struga, bellow the bend (leg. $D$. Pavićević 14/08/1985, 498, n.spec. =1); Valovito jezero Lake (leg. G. Džukić 11/07/1983, 498, n.spec. = 1); (leg. Lj. Tomović, R. Ajtić 01/08/2002, 498, n.spec. = 1); Vučja Jama (leg. G. Džukić 14/09/1985, 498, n.spec. = 1); Zminje jezero Lake (leg. J. Crnobrnja 04/09/1986, 498, n.spec. =1); Serbia Banat Vršački Breg Mt.: Markovac (v.) (leg. J. Rašajski, 01/10/1969, 506, n.spec. = 1); Široko Bilo, $500 \mathrm{~m}$ from the mountain house (leg. B. Božičić 26/05/1985, 506, n.spec. =1); near the forest house (leg. anonim. 09/07/1988, 506, n.spec. = 1); near the mountain house, toward Prevala (leg. $\mathrm{Lj}$. Tomović 02/10/2002, 506, n.spec. = 1); (leg. anonim. 05/06/2009, 506, n.spec. =1); (leg. J. Rašajski s. d., 506, n.spec. =1); Central Serbia Kopaonik Mt.: Pančićev Vrh, Velika Ravan (leg. anonim., 18/07/1987, 502, n.spec. = 1); (leg. Lj. Tomović, R. Ajtić 25/08/2008, 502, n.spec. =1); Suvo Rudište (leg. S. Matvejev 07/09/1954, 502, n.spec. = 1); Žljeb, bellow Žljeb (leg. A. Rohalj 17/07/1987, 502, n.spec. = 1); (leg. K. Vasić 1961, 502, n.spec. =1); (leg. S. Matvejev 24/05/1951, 502, n.spec. =1); Kosovo Šar planina Mts.: Ljuboten Mt. (leg. anonim., 18/06/1953, 501, n.spec. =1); Piribeg Mt., Brezovica (leg. P. Jakšić 20/07/1986, 501, n.spec. =2); Durlov potok (leg. anonim. 03/07/1988, 501, n.spec. = 1); (leg. I. Savić 19/09/1967, 501, n.spec. =1); Metohia Mokra Gora Mt.: Mokra Planina Mt., Savina voda (leg. G. Džukić, 09/06/1977, 500, n.spec. =1); South-eastern Serbia Besna Kobila Mt.: Valozi (leg. Lj. Tomović, R. Ajtić, 16/08/2006, 500, n.spec. =1); South-western Serbia Mokra Gora Mt.: Mokra Planina Mt., Crvene Vode (leg. B. Tatić, s. d., 500, n.spec. =1); Srem Fruška Gora Mt.: Stražilovo (leg. anonim., 1959, 521, n.spec. =1); (leg. M. Marković 01/08/1998, 521, n.spec. = 1); Pećinci: Obrež (v.), Obedska bara pond, Matijevica forest (leg. anonim. 03/07/1988, 
521, n.spec. =1); Ruma: Grabovci (v.) (leg. anonim. August 1989, 521, n.spec. =1); Nikinci (v.) (leg. R. Silaški 21/05/1988, 521, n.spec. = 1); Slovenia Kranj Tržič: Gozd (leg. anonim., 06/07/1991, 370, n.spec. $=1$ ).

\section{Vipera ursinii}

Montenegro Eastern Montenegro Bjelasica Mt.: Lalevića Dolovi (leg. Lj. Tomović, $R$. Ajtić, 2000-2002, 365, n.spec. = 8); Northern Montenegro Durmitor Mt.: Komarnica (r.), Klještina (leg. P. Jakšić, 15/08/1986, 364, n.spec. = 1); Lojanik (leg. anonim. July 1989, 364, n.spec. =1); Šavnik: Kutnja Njiva (v.), Siljevo Brdo hill (leg. M. Džukić 01/08/1984, 364, n.spec. =1); Serbia Metohia Paštrik Mt.: Gorožup, above (leg. G. Džukić, 19/07/1980, 363, n.spec. =1); Prokletije Mts. (leg. M. Radovanović 1954, 363, n.spec. =1).

\section{Analysis of taxa and specimens}

The herpetological collection contains samples of 65 species from 23 countries. These species belong to 19 families ( 3 of Testudines, 8 of Lacertilia and 8 of Serpentes).

\section{TESTUDINES}

Familia Cheloniidae

Caretta caretta - 1 specimen from Montenegro with precise locality.

\section{Familia Emydidae}

Emys orbicularis - 23 specimens from two countries (Serbia and Montenegro) with precise localities.

\section{Familia Testudinidae}

Testudo graeca - 1 specimen from Macedonia with precise locality.

Testudo hermanni - 4 specimens from three countries (Serbia, Macedonia and Montenegro) with precise localities.

\section{LACERTILIA}

\section{Familia Agamidae}

Agama lionotus - 1 specimen from Kenya with precise locality.

Laudakia badakhshana - 4 specimens from Afghanistan with precise localities.

Laudakia caucasica - 6 specimens from Afghanistan with precise localities. 
Stellagama stellio - 1 specimen from Cyprus with unknown locality. localities.

Trapelus ruderatus - 2 specimens from Afghanistan with precise

\section{Familia Anguidae}

Anguis fragilis complex - 299 specimens from six countries (Bosnia \& Herzegovina, Croatia, Macedonia, Montenegro, Serbia and Slovenia) in 254 entries with precise localities, and 45 entries with no data.

Pseudopus apodus - 10 specimens from three countries (Bosnia \& Herzegovina, Macedonia, and Montenegro) with precise localities.

\section{Familia Chamaeleonidae}

Furcifer pardalis - 1 specimen from Mauritius with unknown locality.

\section{Familia Gekkonidae}

Hemidactylus turcicus - 26 specimens from two countries (Croatia and Montenegro) with precise localities.

Mediodactylus kotschyi - 150 specimens from five countries (Bulgaria, Greece, Iraq, Macedonia and Serbia) in 143 entries with precise localities, and 7 entries with no data.

Tenuidactylus fedtschenkoi - 10 specimens from Afghanistan with precise localities.

\section{Familia Lacertidae}

Algyroides nigropunctatus - 119 specimens from five countries (Croatia, Macedonia, Montenegro, Serbia and Slovenia) with precise localities.

Dalmatolacerta oxycephala - 776 specimens from two countries (Croatia and Montenegro) in 759 entries with precise localities, and 17 entries with no data.

Darevskia praticola - 166 specimens from two countries (Russia and Serbia) with precise localities.

Dinarolacerta montenegrina - 8 specimens (the holotype and paratypes) from Montenegro with precise localities.

Dinarolacerta mosorensis - 67 specimens from three countries (Bosnia \& Herzegovina, Croatia and Montenegro) with precise localities.

Eremias sp. - 1 specimen from Afghanistan with precise locality.

Iberolacerta horvathi - 12 specimens from two countries (Croatia and Slovenia) with precise localities. 
Lacerta agilis - 364 specimens from five countries (Bosnia \& Herzegovina, Croatia, Macedonia, Montenegro and Serbia) in 343 entries with precise localities, and 21 entries with no data.

Lacerta trilineata - 24 specimens from five countries (Bosnia \& Herzegovina, Croatia, Greece, Macedonia and Montenegro) in 20 entries with precise localities, and 4 entries with no data.

Lacerta viridis - 358 specimens from six countries (Bosnia \& Herzegovina, Croatia, Macedonia, Montenegro, Serbia and Slovenia) in 348 entries with precise localities, and 10 entries with no data.

Ophisops elegans -1 specimen from Libya with precise locality.

Podarcis erhardii - 179 specimens from two countries (Macedonia and Serbia) with precise localities.

Podarcis melisellensis - 872 specimens from three countries (Bosnia \& Herzegovina, Croatia and Montenegro) in 838 entries with precise localities, and 34 entries with no data.

Podarcis muralis - 2108 specimens from seven countries (Albania, Bosnia \& Herzegovina, Croatia, Macedonia, Montenegro, Serbia and Slovenia) in 1931 entries with precise localities, and 177 entries with no data.

Podarcis peloponnesiacus - 1 specimen from Greece with precise locality.

Podarcis siculus - 629 specimens from five countries (Bosnia \& Herzegovina, Croatia, Italy, Montenegro and Slovenia) in 599 entries with precise localities, and 30 entries with no data.

Podarcis tauricus - 120 specimens from two countries (Macedonia and Serbia) with precise localities.

Zootoca vivipara - 238 specimens from five countries (Bosnia \& Herzegovina, Croatia, Macedonia, Montenegro, Russia and Serbia) in 228 entries with precise localities, and 10 entries with no data.

\section{Familia Phrynosomatidae}

Phrynosoma cornutum - 2 specimens from the USA with precise localities.

\section{Familia Phyllodactylidae}

Tarentola mauritanica -4 specimens from Italy in 4 entries with precise localities. 


\section{Familia Scincidae} localities.

Ablepharus bivittatus - 2 specimens from Afghanistan with precise

Ablepharus kitaibelii - 434 specimens from three countries (Macedonia, Montenegro and Serbia) with precise localities.

Chalcides ocellatus -2 specimens from two countries: Greece - with precise locality and Morocco - without precise locality. locality.

Eurylepis taeniolata - 1 specimen from Afghanistan with precise

Eutropis dissimilis - 1 specimen from Afghanistan with precise locality.

\section{SERPENTES}

\section{Familia Colubridae}

Boiga blandingii - 1 specimen from Liberia with precise locality.

Coronella austriaca - 108 specimens from five countries (Bosnia \& Herzegovina, Croatia, Macedonia, Montenegro and Serbia) in 101 entries with precise localities, and 7 entries with no data.

Dolichophis caspius - 42 specimens from four countries (Macedonia, Montenegro, Romania and Serbia) in 36 entries with precise localities, and 6 entries with no data.

Elaphe quatuorlineata - 6 specimens from two countries (Macedonia and Montenegro) with precise localities.

Hierophis gemonensis - 28 specimens from four countries (Bosnia \& Herzegovina, Croatia, Macedonia and Montenegro) in 24 entries with precise localities, and 4 entries with no data.

Hierophis viridiflavus - 2 specimens from Croatia with precise localities.

Natrix natrix - 194 specimens from seven countries (Bosnia \& Herzegovina, Croatia, Macedonia, Montenegro, Slovakia, Slovenia and Serbia) in 173 entries with precise localities, and 21 entries with no data.

Natrix tessellata - 85 specimens from five countries (Croatia, Macedonia, Montenegro, Slovenia and Serbia) in 77 entries with precise localities, and 8 entries with no data.

Pituophis catenifer - 2 specimens from the USA with precise localities.

Platyceps najadum - 11 specimens from four countries (Bosnia \& Herzegovina, Croatia, Montenegro and Serbia) with precise localities.

Telescopus fallax - 11 specimens from four countries (Bulgaria, Croatia, Macedonia and Montenegro) with precise localities. 
Zamenis longissimus - 115 specimens from five countries (Bosnia \& Herzegovina, Croatia, Macedonia, Montenegro and Serbia) in 109 entries with precise localities, and 6 entries with no data.

Zamenis situla - 7 specimens from two countries (Croatia and Macedonia) in 5 entries with precise localities, and 2 entries with no data.

\section{Familia Elapidae}

Micrurus sp. -1 specimen from Venezuela with precise locality.

\section{Familia Erycidae}

Eryx jaculus - 1 specimen from Macedonia with precise locality.

\section{Familia Lamprophiidae}

Atractaspis sp. -1 specimen from Liberia with precise locality.

Boaedon fuliginosus - 1 specimen from Liberia with precise locality.

Lycophidion sp. -1 specimen from Liberia with precise locality.

Malpolon insignitus - 17 specimens from five countries (Bosnia \& Herzegovina, Croatia, Libya, Macedonia and Montenegro) in 16 entries with precise localities, and 1 entry with no data.

\section{Familia Typhlopidae}

Xerotyphlops vermicularis - 22 specimens from two countries (Macedonia and Montenegro) with precise localities.

\section{Familia Viperidae}

Atheris squamigera - 1 specimen from Kenya with precise locality.

Bitis gabonica - 1 specimen from Liberia with precise locality.

Vipera ammodytes - 460 specimens from seven countries (Bosnia \& Herzegovina, Bulgaria, Croatia, Macedonia, Montenegro, Slovenia and Serbia) in 425 entries with precise localities, and 35 entries with no data.

Vipera berus - 55 specimens from six countries (Bosnia \& Herzegovina, Croatia, Macedonia, Montenegro, Slovenia and Serbia) in 54 entries with precise localities, and 1 entry with no data.

Vipera ursinii - 13 specimens from two countries (Montenegro and Serbia) with precise localities. 


\section{DISCUSSION}

The Herpetological Collection stored at the Institute for Biological research "Siniša Stanković", University of Belgrade, includes 8213 specimens belonging to 65 species and 19 families. The best-represented families are Lacertidae $(6043-73.6 \%)$, Colubridae $(612-7.5 \%)$ and Viperidae $(530-6.5 \%)$, while Cheloniidae, Chamaeleonidae, Phrynosomatidae, Erycidae and Elapidae are represented only with one or two specimens. All species of Serbian herpetofauna (Tomović et al. 2014) and most reptilian species characteristic for the Balkan Peninsula are present in the collection.

This collection is distinctive because many of the species are covered with large population samples. Particularly interesting are the samples of populations exhibiting important evolutionary and/or biogeographical phenomena (Džukić \& Kalezić 2004) such as endemicity, range marginality, intraspecific diversity, intergradation and hybridisation, variation in reproductive modes, range dynamism, heterogeneity and mosaicism. Significant are also the specimens or large population samples of endemic or subendemic species: Algyroides nigropunctatus (Džukić \& Pasuljević 1979), Dalmatolacerta oxycephala (Podnar et al. 2014), Dinarolacerta montenegrina (Ljubisavljević et al. 2007), Dinarolacerta mosorensis (Ljubisavljević et al. 2016) and Podarcis melisellensis (Podnar et al. 2004). Samples from the contact zones of the newly described species belonging to the Anguis fragilis complex (Jablonski et al. 2016) provide the opportunity for future research of precise geographic distribution of the taxa belonging to this species complex. In addition, there are samples of the peripheral populations of: Darevskia praticola (Ljubisavljević et al. 2008), Podarcis erhardii (Tomović et al. 2014, Urošević et al. 2015), P. tauricus (Ljubisavljević et al. 2010a), Zootoca vivipara (Ljubisavljević et al. 2010b), Vipera ammodytes (Jelić et al. 2013), as well as samples from the isolated populations of species with fragmented ranges: Dolichophis caspius, Vipera berus, V. ursinii (Tomović et al. 2014, 2015a, b). Many of the distribution data from the collection were used to estimate conservation statuses of the Serbian reptiles (Tomović et al. 2015a, b). The holotype specimen and seven paratype specimens of the newly described species Dinarolacerta montenegrina (Ljubisavljević et al. 2007) are among the most valuable samples contained within this herpetological collection.

The collection is important due to its taxonomical and geographical richness as well as the presence of large population samples of species-series. On a par with its historical value, the Herpetological collection of the Institute for Biological Research "Siniša Stanković" University of Belgrade, stores data which are exceptionally valuable in the research opportunities they provide for the studies of systematics, biogeography, 
ecology, morphology, conservation and many other aspects of the biology of reptiles. Some of the older collection samples could be very valuable for future research, since habitats of several species are under increased anthropogenic pressures including pollution, disturbance and habitat destruction, and they could be used to assess the zero state. New technologies, such as micro-CT scanning, could facilitate more efficient morphological analyses and sharing of the shape data with other institutions, avoiding the risk of degradation, harm or loss of the original material, especially the type specimens or samples of historical value.

Although it is neither as old nor as rich as some other zoological collections (Boulenger 1889, Milto \& Barabanov 2011), the significance of the collection publicized herein is substantial. To our knowledge, not many publications exist regarding similar collections in the neighboring countries (e.g. Mekinić et al. 2013). However, numerous museums, including some from the region, made their collections available online (e.g. www. prirodoslovni.com/inventarna/en). This is not surprising, because digitalization of natural history collections started already in the 1970s (Graham et al. 2004), and there are attempts at making a transnational network of museum repositories (www.biocase.org). Nevertheless, the importance of the existing biological collections (storing billions of specimens - Graham et al. 2004) rises in modern times, when we witness both the extinctions and discoveries of numerous species and various environmental stresses at the global level (Winker 2004, Garg et al. 2017). A recent illustration of how important collections can be is the loss of one (Warrell et al. 2010). Also, despite the fast development of new technologies and sometimes strong opposition to collecting of specimens from the wild, the idea that voucher specimens could be replaced with photos, recordings and tissue samples was decisively refuted (Dubois 2017, Garraffoni \& Freitas 2017, Minteer et al. 2014, Rocha et al. 2014, Rogers et al. 2017). For all those reasons, we feel that this collection shall contribute to the wider herpetological community. It has already been used for several publications (see above), and its potential is yet to be fully appreciated (McLean et al. 2016).

\section{Acknowledgements}

This research was partly financed by the Ministry of Education, Science and Technological Development of Republic of Serbia (Grant No. 173043). For great help in making an inventory of the Herpetological collection, we would like to thank our colleagues Katarina Ljubisavljević, Tanja Vukov and Ana Golubović, together with the following students of the Faculty of Biology: Ana Cvetković, Ana Kijanović, Ana Rašković, Dušan Stojanović, 
Jelena Jovović, Katarina Samardžić, Maja Čabrilo, Milica Trajković, Neda Bogdanović, Stefan Avramović and Uroš Ilić.

\section{REFERENCES}

Boulenger, G. A. (1889): Catalogue of Chelonians, Rhynchocephalians and Crocodiles in the British Museum (Natural History). - Taylor \& Francis, London.

Dubois, A. (2017): The need for reference specimens in zoological taxonomy and nomenclature. - Bionomina 12: 4-38.

Džukić, G., Pasuljević, G. (1979): O rasprostranjenju ljuskavog guštera Algyroides nigropunctatus (Dumeril et Bibron, 1839) (Reptilia, Lacertidae). - Biosistematika 5: 61-70. In Serbian with English summary.

Džukić, G., Kalezić, M. L. (2004): The Biodiversity of Amphibians and Reptiles in the Balkan Peninsula. In: Griffiths, H. I., Kryštufek, B., Reed, J. M. (ed.): Balkan Biodiversity: Pattern and Process in the European Hotspot: 167-192. Kluwer Academic Publishers, Dordrecht, Netherlands.

Džukić, G., Cvijanović, M., Urošević, A., Vukov, T., Tomašević-Kolarov, N., Slijepčević, M., Ivanović, A., Kalezić, M. L. (2015): The batrachological collections of the Institute for Biological Research "Siniša Stanković", University of Belgrade. - Bulletin of the Natural History Museum 8: 118-167.

Garg, S., Suyesh, R., Sukesan, S., Biju, S. D. (2017): Seven new species of Night Frogs (Anura, Nyctibatrachidae) from the Western Ghats Biodiversity Hotspot of India, with remarkably high diversity of diminutive forms. - PeerJ 5: e3007.

Garraffoni, A. R. S., Freitas, A. V. L. (2017): Photos belong in the taxonomic Code. - Science 355: 805.

Graham, C. H., Ferrier, S., Huettman, F., Moritz, C., Townsend Peterson, A. (2004): New developments in museum-based informatics and applications in biodiversity analysis. - Trends in Ecology and Evolution 19: 497-503.

Jablonski, D., Jandzik, D., Mikulíček, P., Džukić, G., Ljubisavljević, K., Tzankov, N., Jelić, D., Thanou, E., Moravec, J., Gvoždík, V. (2016): Contrasting evolutionary histories of the legless lizards slow worms (Anguis) shaped by the topography of the Balkan Peninsula. - BMC Evolutionary Biology 16: 99.

Jelić, D., Ajtić, R., Sterijovski, B., Crnobrnja-Isailović, J., Lelo, S., Tomović, Lj. (2013): Distribution of the genus Vipera in the western and central Balkans. Herpetozoa 25: 109-132.

Ljubisavljević, K., Arribas, O., Džukić, G., Carranza, S. (2007): Genetic and morphological differentiation of Mosor rock lizards, Dinarolacerta mosorensis (Kolombatović, 1886), with the description of a new species from the Prokletije Mountain Massif (Montenegro) (Squamata: Lacertidae). - Zootaxa 1613: 1-22.

Ljubisavljević, K., Džukić, G., Kalezić, M. L. (2008): Female reproductive life history traits of the meadow lizard, Darevskia praticola (Eversmann, 1834) 
(Squamata: Lacertidae) from the westernmost portion of the species range. Polish Journal of Ecology 56: 289-297.

Ljubisavljević, K., Džukić, G., Kalezić, M. L. (2010a): Female reproductive characteristics of the Balkan wall lizard (Podarcis taurica) in the northwestern periphery of its range. - Central European Journal of Biology 5: 391-395.

Ljubisavljević, K., Jović, D., Džukić, G. (2010b): Morphological variation of the common lizard (Zootoca vivipara Jacquin, 1787) in the Central Balkan. Archives of Biological Sciences 62: 791-799.

Ljubisavljević, K., Polović, L., Iković, V., Vuksanović, S., Zagora, V., Urošević, A., Tomović, Lj. (2016). New records and updated distribution of the endemic Balkan rock lizards Dinarolacerta spp. in Montenegro. - Ecologica Montenegrina 9: 46-50.

Mekinić, S., Boban, J., Vladović, D., Cvitanić, R., Ževrnja, N. (2013): Amphibians in herpetological collection of Natural History Museum Split, Croatia. - Natura Montenegrina 12: 593-597.

Milto, K. D., Barabanov, A. V. (2011): An annotated catalogue of the amphibian types in the collection of the Zoological Institute, Russian Academy of Sciences, St. Petersburg. - Russian Journal of Herpetology 18: 137-153.

Minteer, B. A., Collins, J. P., Love, K. E., Puschendorf, R. (2014): Avoiding (Re)extinction. - Science 344: 260.

McLean, B. S., Bell, K. C., Dunnum, J. L., Abrahamson, B., Colella, J. P., Deardorff, E. R., Weber, J. A., Jones, A. K., Salazar-Miralles, F., Cook, J. A. (2016): Natural history collections-based research: progress, promise, and best practices. - Journal of Mammalogy 97: 287-297.

Podnar, M., Mayer, W., Tvrtković, N. (2004): Mitochondrial phylogeography of the Dalmatian wall lizard, Podarcis melisellensis (Lacertidae). - Organisms Diversity \& Evolution 4: 307-317.

Podnar, M., Bruvo Mađarić, B., Mayer, W. (2014): Non-concordant phylogeographical patterns of three widely codistributed endemic Western Balkans lacertid lizards (Reptilia, Lacertidae) shaped by specific habitat requirements and different responses to Pleistocene climatic oscillations. - Journal of Zoological Systematics and Evolutionary Research 52: 119-129.

Rocha, L. A., Aleixo, A., Allen, G., Almeda, F., Baldwin, C. C. et al. (2014): Specimen collection: An essential tool. - Science 344: 814-815.

Rogers, D. C., Ahyong, S. T., Boyko, C. B., D'Acoz, C. D. et al. (2017): Images are not and should not ever be type specimens: a rebuttal to Garraffoni \& Freitas. - Zootaxa 4269: 455-459.

Sillero, N., Campos, J., Bonardi, A., Corti, C., Creemers, R., Crochet, P.-A., Crnobrnja-Isailović, J., Denoël, M., Ficetola, G. F., Gonçalves, J., Kuzmin, S., Lymberakis, P., de Pous, P., Rodríguez, A., Sindaco, R., Speybroeck, J., Toxopeus, B., Vieites, D. R., Vences, M. (2014): Updated distribution and biogeography of amphibians and reptiles of Europe.-Amphibia-Reptilia 35: 1-31.

Tomović, Lj., Ajtić, R., Ljubisavljević, K., Urošević, A., Jović, D., Krizmanić, I., Labus, N., Đorđević, S., Kalezić, M. L., Vukov, T., Džukić, G. (2014): 
Reptiles in Serbia - distribution and diversity patterns. - Bulletin of the Natural History Museum 7: 129-158.

Tomović, Lj. Kalezić, M., Džukić, G. (Eds.) (2015a): Crvena knjiga faune Srbije II: Gmizavci. - Univerzitet u Beogradu-Biološki fakultet \& Zavod za zaštitu prirode Srbije, Beograd.

Tomović, Lj., Urošević, A., Vukov, T., Ajtić, R., Ljubisavljević, K., Krizmanić, I., Jović, D., Labus, N., Đorđević, S., Kalezić, M. L., Džukić, G., Luiselli, L. (2015b): Threatening levels and extinction risks based on distributional, ecological and life-history datasets (DELH) versus IUCN criteria: example of Serbian reptiles. - Biodiversity and Conservation 24: 2913-2934.

Uetz, P., Freed, P. \& Hošek, J. (Eds.) (2017): The Reptile Database, http://www. reptile-database.org. Accessed on 08.01.2017.

Urošević, A., Ljubisavljević, K., Tomović, Lj., Krizmanić, I., Ajtić, R., Simović, A., Labus, N., Jović, D., Golubović, A., Anđelković, M., Džukić, G. (2015): Contribution to the knowledge of distribution and diversity of lacertid lizards in Serbia. - Ecologica Montenegrina 2: 197-227.

Warrell, D. A., Theakston, R. D. G., Wüster, W. (2010): Destruction of the collection of reptiles and arthropods at Butantan Institute: a view from the United Kingdom. - The Journal of Venomous Animals and Toxins including Tropical Diseases 16: 534-536.

Winker, K. (2004): Natural History Museums in a Postbiodiversity Era. BioScience 54: 455-459.

\section{SUPPORTING INFORMATION}

Online Appendix - List of Reptile specimens deposed in the Herpetological collection with collection numbers, names of species, original locations, reconstructed and classified exact locations, date of collection, legators, numbers of specimens and sub-collections.

\section{ABBREVIATIONS IN THE CATALOGUE AND APPENDIX}

s. I. - sine loco (unknown place of collection)

s. d. - sine die (unknown date of collection)

h. - hamlet

i. - island

r. - river

s. - skerry

v. - village 
104 DŽUKić, G. ET AL.: Herpetological COLleCtion - InSTITUTE “S. STANKOVIĆ”

ХЕРПЕТОЛОШКА ЗБИРКА ИНСТИТУТА ЗА БИОЛОШКА ИСТРАЖИВАњА „СИНИША СТАНКОВИЋ“, УНИВЕРЗИТЕТА У БЕОГРАДУ

\author{
ГЕОРГ ЏУКИЋ, ЉИЉАНА ТОМОВИЋ, МАРКО АНЪЕЛКОВИЋ, \\ АЛЕКСАНДАР УРОШЕВИЋ, СОЊА НИКОЛИЋ, МИЛОШ КАЛЕЗИЋ
}

\title{
Р Е 3 И М Е
}

Овај рад представља попис врста гмизаваца депонованих у херпетолошкој збирци Института за Биолошка Истраживања „Синиша Станковић“, Универзитета у Београду. Збирка се састоји од 8213 примерака 65 врста гмизаваца сакупљених из 23 земље. Највећи део збирке садржи материјал са територије бивше Југославије. Врсте заступљене у збирци класификоване су у оквиру 19 породица (три у оквиру Testudines, осам у оквиру Lacertilia и осам у оквиру Serpentes). По броју примерака, најзаступљеније су породице Lacertidae (6043 - 73,6 \%), Colubridae $(612-7,5 \%)$ и Viperidae $(530-6,5 \%)$, док су породице Cheloniidae, Chamaeleonidae, Phrynosomatidae, Erycidae и Elapidae заступљене са по једним или два примерка. Примерци свих врста гмизаваца који живе у Србији постоје у збирци, као и већина врста карактеристичних за Балканско полуострво. Специфичан значај збирке огледа се у великим популационим узорцима неколико врста. Осим историјског значаја, посебна вредност херпетолошке збирке огледа се у томе што депонован материјал омогућава студије систематике, дистрибуције, екологије, морфологије, конзервационе биологије као и различитих еволуционих и биогеографских феномена (нпр. ендемизма, интраспецијског диверзитета, хибридизације таксона, итд). 